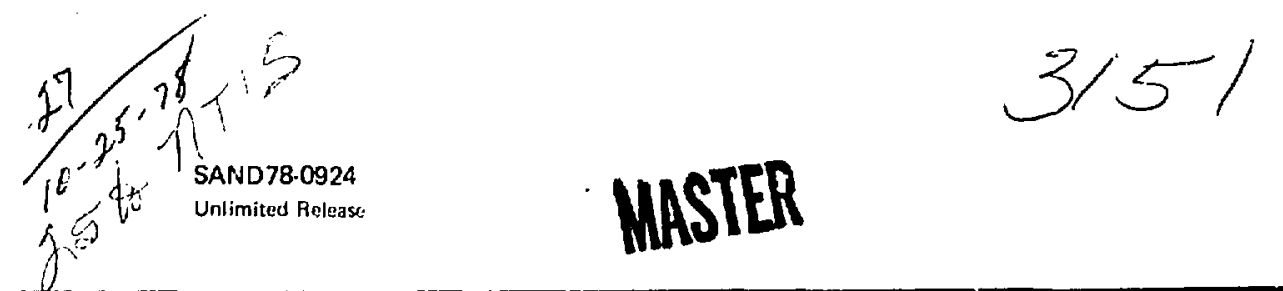

\title{
Predicted Temperatures in a Bedded-Salt Repository Resulting From Burial of DOE High-Level Nuclear Waste Canisters
}

Carl E. Siston 
SA.Vก7ด-0924

linlimited Releaso

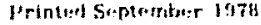

Work Complated lebruary 1 id

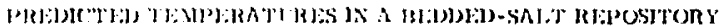
t)

WATI: CANSTH:14:
\end{abstract}

Carl K. Sisson

Flatil Mechanics $\star$ Jleat Trunster Division 1262

Sandia l-aboraturies

Alluquerque, New stexico ag185

\title{
AHSTINCT
}

A 2-D thermal nodel is defined and used to calculate temperatures within support pillars of a bedded salt repository. 'The particular configuration considered includes UOE high-level waste canisters (producing $300 \mathrm{~W}$ each) buried threc abreast below the Hoor of a 20-loot wide drift.

\begin{abstract}
A 3-D "elose-in" thermal model is also defined and used to eotimate the the rmal response of bedded sall near the buried canisters. This model uses boundary conditions from the 2-D "global" model.
\end{abstract}

This work is supported by the L:S. Department of Energy. 


\section{coNTh.XTS}

1. Jatrodiction

11. Welinition of Hurial Configuration ant Basic Colstraints of St tudy $i$

III. Thermal Xodel Cireometry u

IV. Heundury Conditions 3

V. Vuterial Properties

VI. Volumetric lleating in Nuclear Waste

ilf. Nuserical Calculations

$\begin{array}{lr}\text { Vlli. Temperature Hesults } & 10\end{array}$

LS. Cencluding Remarks 12

lifeferi:ncess

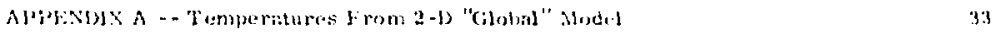

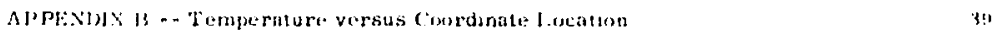

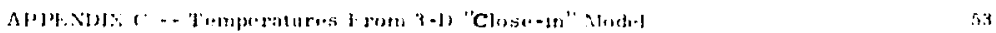

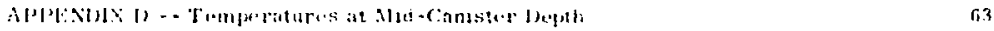




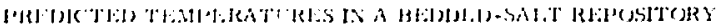

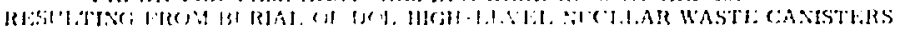

\section{Introducition}

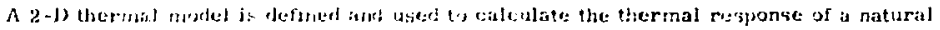

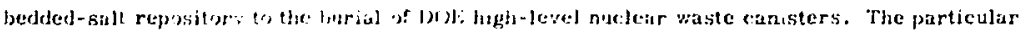

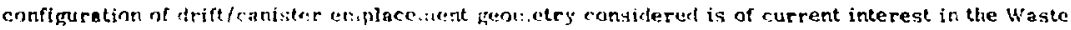
Isoletion [ji]nt ['lent (W[J'l) progra:m-rlevelopment effort. The thermal-response calculations were requested for use in temuralure-ieperifent creep studies. (of particular interest ore the temperatures in the berited salt surrounding the access drift $(* . .$. in the pillars).

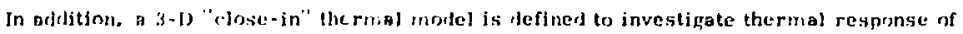
the bededed salt close to the buried caristers. This "clnse-in" anded uses boundary conditions from the 2-D "globrl" nodel. The primory result desircu from this 3-1) model is the prat teanperature in the hededed salt. However, the Jetailed results nay also be of interest in caninter buoyency and whter-migention atulies.

This report discusacs the difi/coninter burial confinurotion, dofines the thermal ancklels, ond proviles an oyerview of the tempen ature results. Completo temperature results are retained on magnetic tape for ase in other englneering studies.

\section{Definition of Burial Configuration and Basic Constraints of Sludy}

The following information was provided to describe the inift/waste canister burial geometry and the basic constroints for the thernal study:

- Drift dinensions $\sim 20$ leet (width) by 15 feet (height).

- Pillar widtl $\sim 80$ feet.

- Thermal heating $-3-300 W$ canisters spaced 5 feet apart across drift, at 5-foot intervals along urift.

- Canisters geometry $\sim 20$-foot diameter by 10-foot length buried 10 feet bclow drift Hoor.

- Bedded salt thermal properties per Reference $z$.

- Waste heat decay curve--See Figure 8.

- Time duration of interest $\sim 50$ years. 
'The thermal mode: will consider diffusion effests in a single homogeneous material (salt). No attempt will be mnic to morlel the effect of aquifers, layered geologic material stratifications.

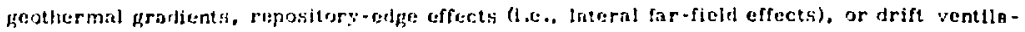

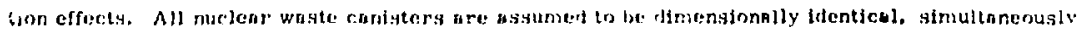

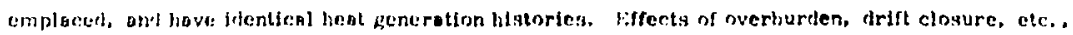
are not constetered ln this thuty.

\section{III. [herma] Morie] Geometry}

Figure 1 showg on elevation view of the drift with the three conisters burled below the drift floor. A plan view of Jrifts, plliers, and canister emplecement geometry is shown in Figure 2. Note thot cross-drifte (service tunnels) ore not shown. For the current study, it is assumed thet such service tunnels will not signiticantly alter temperaturce. Thus, as implied in Figure 2, we are considering an extensive pettern of Urifts. directing intr-rost toward a region enclosed by adiabetic bounderies. Since all canisterg are assumed to be emplaced simultanecusly, adibisatic boundarics are defined by Flgure 2 . The shaded region $(2.5$ feet by 50 feet), containing $1 / 4-$ conister plus 1/2-cenister volumes, defines the portion included in the thermol model.

The prunary purpose of tie thermal model is tes estimate pillar temperatures for use in temperature-dejendent creep studies, 'lo obtain pillar temperatures, il e-I) "global". model is defined (1.igures $3 \mathrm{nnd} 4$ ). In adrition. a 3-D "close-in" model is defined (Figures 5 and $C$ ) to invegtigate thermid response of the bed ded salt close to the buriet canisters. This "close-in" model uses lounilary temperatures from the 2-D "glohal" model.

The vertical dimeasions of the 2-D thermal model were selected arbitrarily (Figure 3 ). Since these are assumed to be adjabatic bounderles, temperature results must be reviewed to validate the assumption (see Section IV. Boundary Conditions, and Section DX, Concluding Remarks).

Figures 3 through 6 slow all thermal model dimensions, geometric subdivisions, node numbering to define lumped-mass diffusion nodes, and the relationship between the 2-D "global" madel and 3-D "close-In" model (sce Section VII. Numerical Calculations).

Tahles I and II list coordinates of node centers for the 2-D and 3-D thermal models.

\section{Boundary Conditions}

As noted in Figures 3 and 5 , most of the model surfaces are adiabatic. For the vertical surfaces (except drift wall). the adiabatic conditions arise from the choice of model boundaries (Figure 2). However, at the upper ond lower bounding surfaces $(z=700$ and $z=-800)$, the adiabatic boundaries ere assumed. For an exact repregentation, these model bounds would ha:e to extend upward to the earth's surface (with some real boundary conditica applied there) and downward, possibly to the earth's geometric center (where an adiabatic boundary might truly 
apply). Ixtending the modal to such hounds would certainly volate the hor:ogeneous material restraint and would surely be impractical for numerical solution. Accepting the adiabutic upper

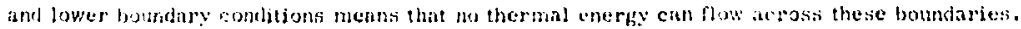

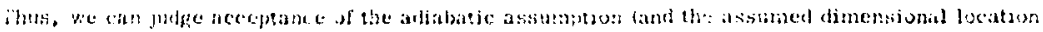

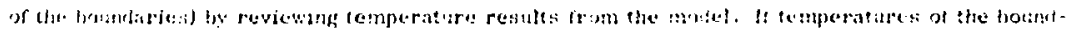

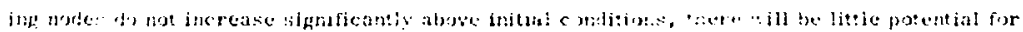

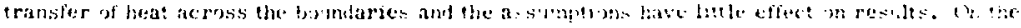

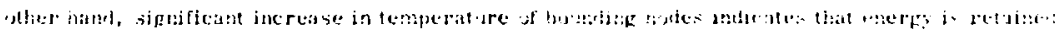

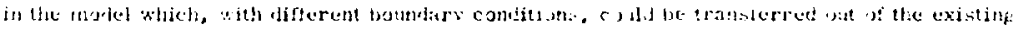
andel.

For wall, ceiling, and flnor surfaces of the trift, the atiotatic assuntion is reasonable

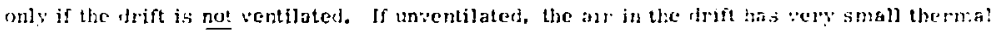

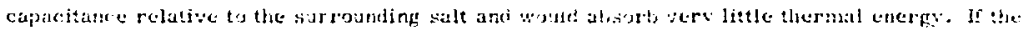

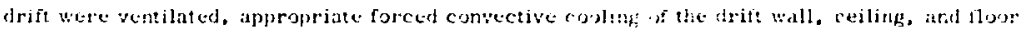
surfoges would have to be ansideret.

\section{Yiafcraj troperties}

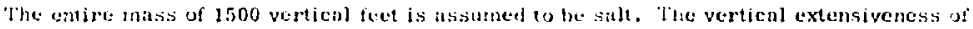

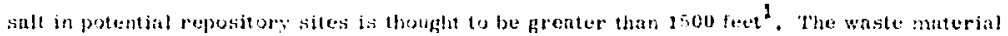
emplaced and the crushed salt back-fill are not treated starately. This simplification of the 2-1)

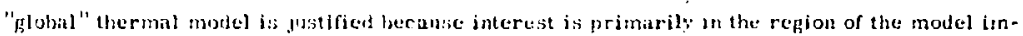
merlintely surroumling the crift ant includes the pillar. For the $\because-1)$ "close-in" thermal mortel. the all-silt assimption is a siniplification mate to improve easc of modeling. Copocitance of the waste and the bediderl salt are essentially the sume. Capacitance of the crushed salt is bout one-fourth that of the bedied salt, but comprises a very small part of the model. The thermel propertics of bedded solt wre taken from Reference 2 and are shown by figures 3 and $r$.

\section{Volumetric lleating in Nuclear Wastt:}

13y definition, the emplaced muclear waste $\mathrm{will}$ generate $300 \mathrm{H} /$ canister at in of emplacement. This heat generation rate decays with time in accorclance with Figure 8 . Waste heat, a tota' of $(3 / 4$ canister $) \times(300 \mathrm{~W} /$ canister $)=225 \mathrm{~W}$, is input to model nodes as indicated by Figures 4 anc 6 .

\section{Numerical Calculations}

Thermal model geometries were discussed easlier and are shown with nose numbering in Figures 3 through 6 : Actual calculation of the lumped-mass diffusion node networks for the 2-D and 3-D thermal models was performed using IIT..1S1 ${ }^{3,4}$ which formalted the networks for solution using CINDA ${ }^{5,6}$. The 2-D "global" thermal model consists of 138 diffusion nodes and 248 conductors. The 3-D "close-jn" thermal model consists of 360 diffusion nojes, 53 boundary nodes, 
and 93r, corducturs. Variable conductivity is consideredm-all conductances were recalculated at each printout time interunl (defined helow to account for temperature variation.

The thermat network solutions employed an implicit finite-difference numerical solution reliemis ${ }^{7}$ with:

- All node temporatires Initiallzed at $0.0^{\circ} \mathrm{C}$.

- Cinlculational time interval

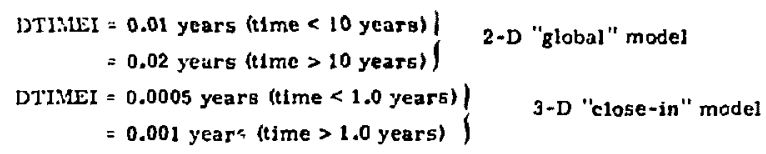

- Hata output intervals

$$
\begin{aligned}
& \text { OCTPYT }=0.1 \text { years (time }<0.5 \text { yearsl } \mid 2-0 \text { "global" model } \\
& =0.5 \text { years (time }>0.5 \text { years) } \\
& \text { OUTPCT }=0.05 \text { years (lime }<0.5 \text { years) } \\
& =0.10 \text { years }(0.5<\tau<1.0 \text { years) } \\
& 0.25 \text { years }(1.0<\tau<3.0 \text { years }) \\
& \text { 3-1) "close-in" model } \\
& =0.50 \text { years (time }>3.0 \text { yenrs) }
\end{aligned}
$$

- Time of solution

0 to 50 ycar: for $2+1$ " global" model

0 to $10+$ years for $3-10$ "close-in" mode!

Calculntional time intervals for the 3-D model were required to be very small to avold numerical instability - mosty because of the small nodes in model regions where temperature gradients werc small. Ilaving this solution, future models would certinly be improved.

During the 2-D model solution, energy input to the model and energy stored in the model were summed for comparison. The values were equal for the full solution, indicating goud energy balance. For the 3-D solution unly the energy input was summed (for comparison with 2-D solution). An energy balance for the 3-D model is difricult with time-varying temperature boundaries.

\section{Temperature Results}

All temperature results from the thermal model are increases above ambient conditions (because initial conditions of $0^{\circ} \mathrm{C}$ were used), where ambient level temperatures are those existing without the heat generated by nuclear waste materials. The geothermal gradient is one source of ambient temperature level. Ambient levels could also be altered by mine ventilation. by heat generated by machines operating in the mine, or by aquifers which might alter heat transfer rates or cause transport of thermal energy. 


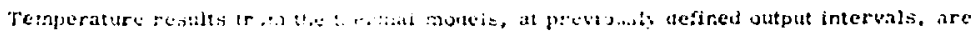

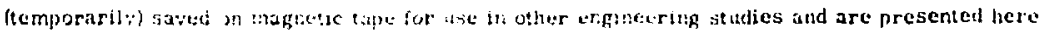

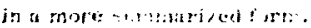

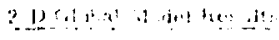

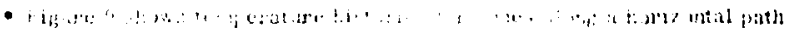

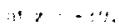

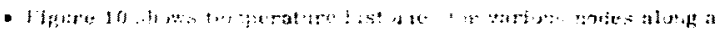

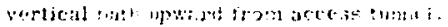

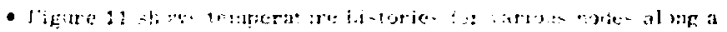
vertical fintl: tontwarel frum accest turne!.

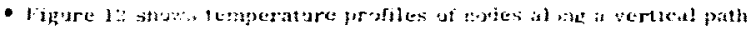
an feet ofi the : 'manel ecentarline.

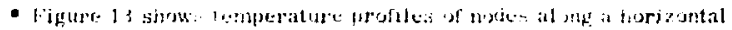
path at : : - 30 .

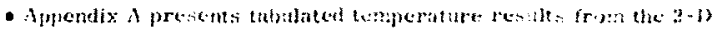

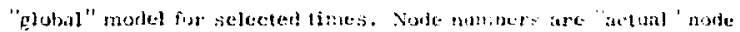

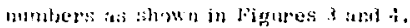

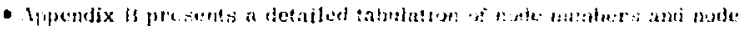

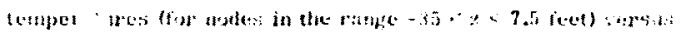
cosorinate locatiesn of mole centers.
}

\section{3-1] Hosu-In Thermal Bodel llesults}

- Figure 14 shows the peak temperature history of bedded sall which jccurs for notle $24.3\left(x-0.98, y-0.272, z^{-}-30\right)$.

- Figures 15 through 17 show tenaperature proliles of nodes along horizontid paths near fore and aft sitriaces $(x+0$ and $x=2.50)$ at $z=-30$. Superimposed on these figures (dasined lines) are the corresiondiog itorizontal profilus from figure 13. Differences in temperature are believed to result from the differences in node sizes. The smaller nodes in the 3-D inodel heat up faster and are assigned the corresponding smaller conductivity (Figure 7 ). Reduced conductivities result in more thermal cenerg, being retained.

- Appendix 1 presents tabulated temperature results from the 3-0 "close in" mocel for selested times. Yode numbers shoun are "relative" node numbers substituted during solution. "Actual" node numbers (as used in Figures 5 and 0 are related to "relative" node numbers by the initial tabulation of Appendix $\mathrm{C}$. 
- Appendix D gives a graphical presentation of node temperatures at midcanlfter depth $(z=-30$ (eet) for selected times.

\section{Concluding Hemarks}

"lhe liermal-response informntian obtained from the 2-D "globul" and 3-D "elose-in" models are belfeved to be sufflelently accirate for preliminary elestgn studies. The mnjor restrictions imposed on the uac of the temperature results are those discussed in Section II -- the problem Jefinition.' However, since the ihormal models are not idealized, sorre noteworthy comments are presented in the paragraphs below.

The comperison (Figures 15 through 17 ) of 2-D and 3-D regults Indicates a difference in energy stor age in the region of the canisters. However, the argument that such differences resinlt from node sizing and varlable conductivity interactiong appears valid. The pillar temperatures from the 2-D model might be slightly eltered if a more deteiled noding were !rled.

The assumptions regarding location and boundary conditions at upper and lower extremes of the 2-D model must be reviewed. At 50 years, the note temperatures were $1.9^{\circ} \mathrm{C}$ at top $(x=700)$ of model and $1.5^{\circ} \mathrm{C}$ at hottom $(\mathrm{z}=-\mathbf{3 0 0}$ ) of moasl. Thus, the model could be improved by $c$ rtending upper and :ower hountary locntions. Jhese non-zero temperatures imply retention of enerry: that is, enerby which could have diffused beyond the selected tipper and bower boundary Jocations. llowever, at 30 year's, these "end" noic temperatures are almust neglifible and pillar temperatures are essentially the same as for 50 years. Thus, peak pillar temperatures are essentially unaffected, althougl: 50-year tempernttres could be slightly high (maybe $1^{\circ} \mathrm{C}$ ).

Some concern must be directed to choice of initial conditions. In particular, the waste material (at time of emplacenent) will truly be at some temper ature greater than the (implied) ambient of $32^{\circ} \mathrm{C}$. Thus, an initial temperature profile would inciude a step at the canister/salt interface. However, the additional energy of concerı should be small compared to the total heat generated over the first year of birial history. Consideration of higher initial waste temperatures would not significantly alter peak-pillar temperature or peak-salt temperature but might slightly alter their time of occurrence. 


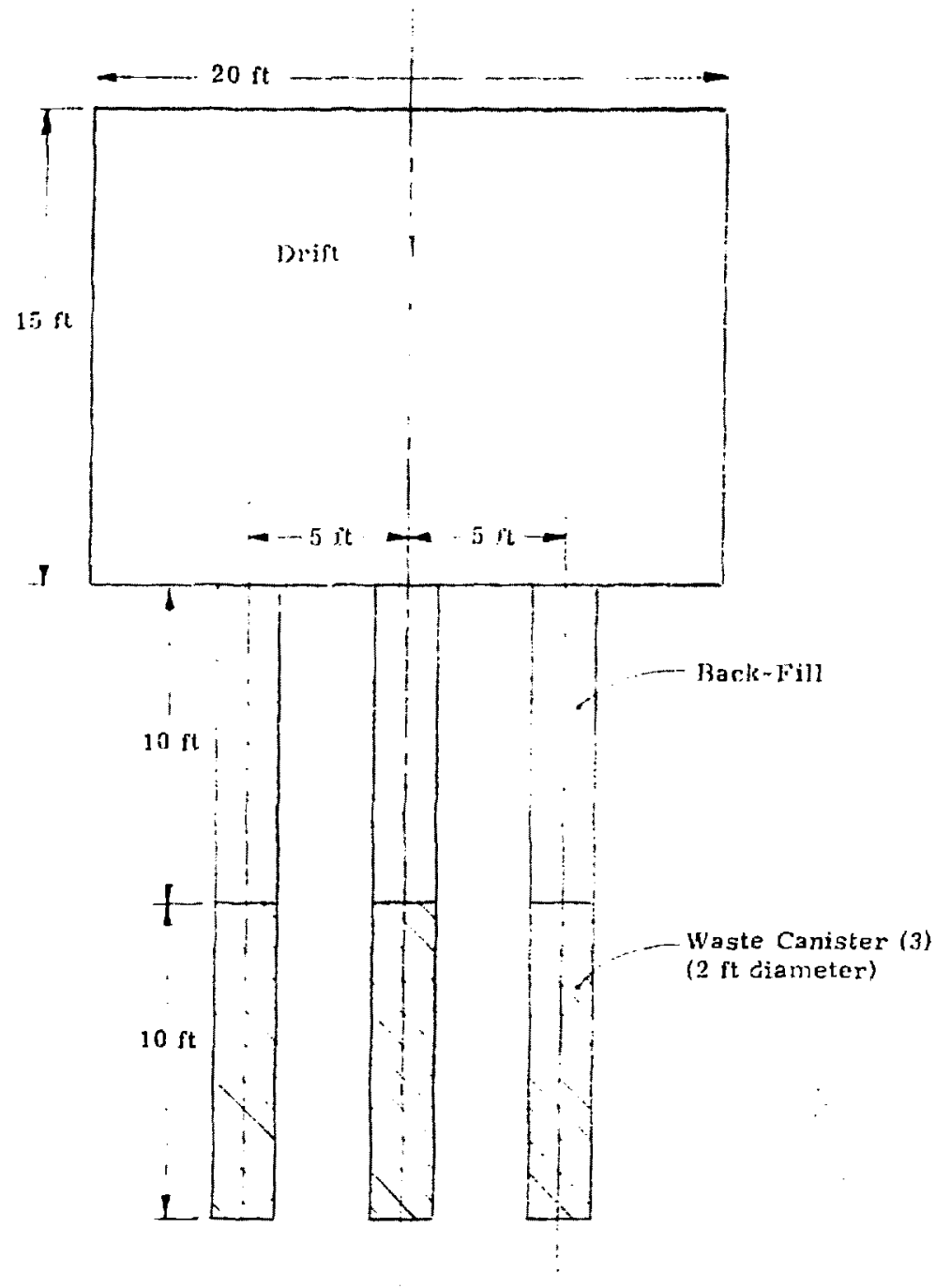

Figure 1. Elevation View Normal to Drift

Showing Waste Carister Burial Geometry 


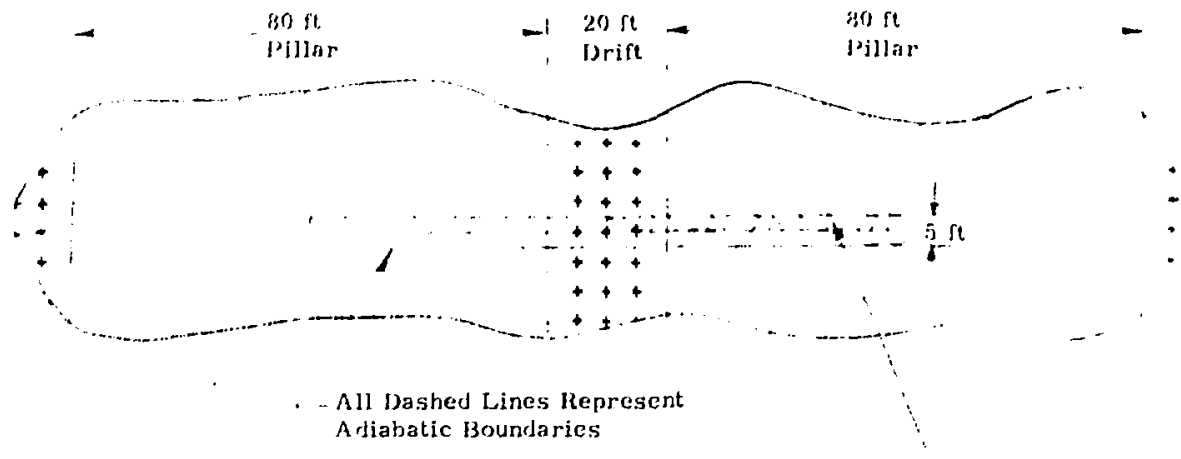

Sharderd Aica Represants -

portion consilered in

2-D) Thermal Nodnel (filobal)

(a. $5 \mathrm{se} b y$ in $\mathrm{st})$

Calculation of leak Thermal Finergy density:

(300 w/canister) (4 canisters)/(500 $\left.\mathrm{rt}^{2}\right)\left(1\right.$ acre $\left./ 43560 \mathrm{ft}^{2}\right)$

$=78.4 \mathrm{kw} / \mathrm{acre}$

Figure 2. Plan View of Drift and Canister Emplacement Teometry 


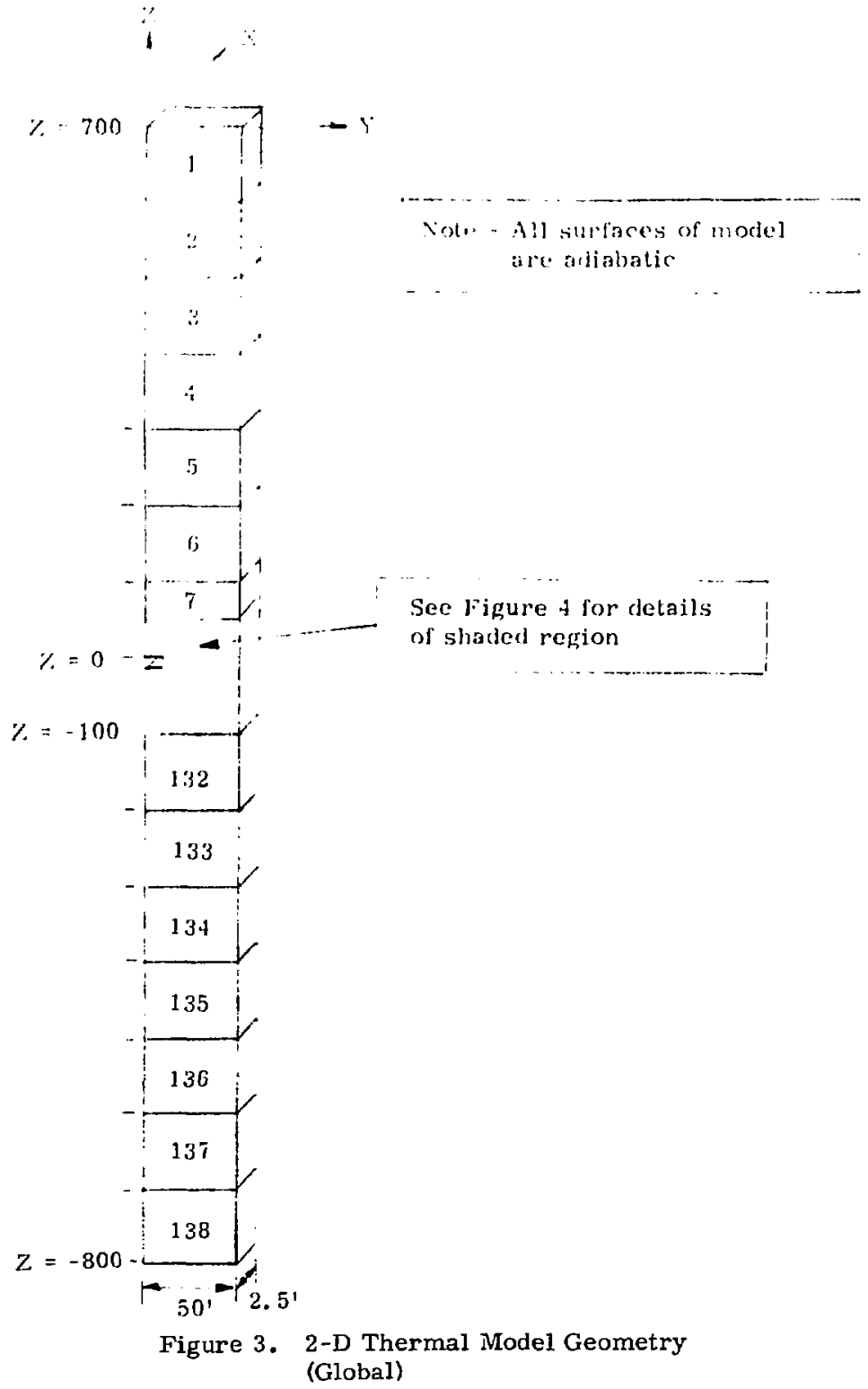




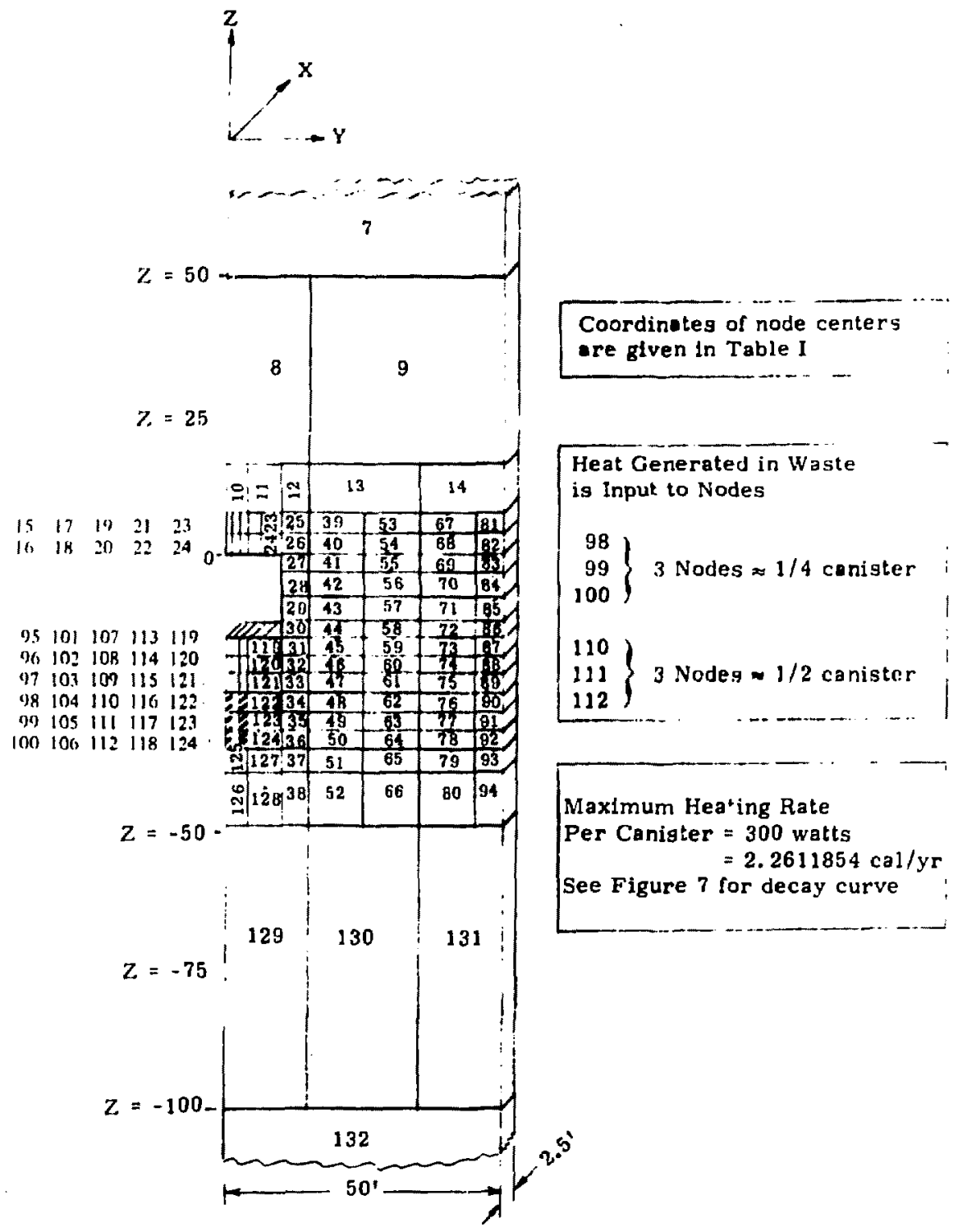

Figure 4. Additional Details of 2-D Thermal Model Geometry for Global Solution 


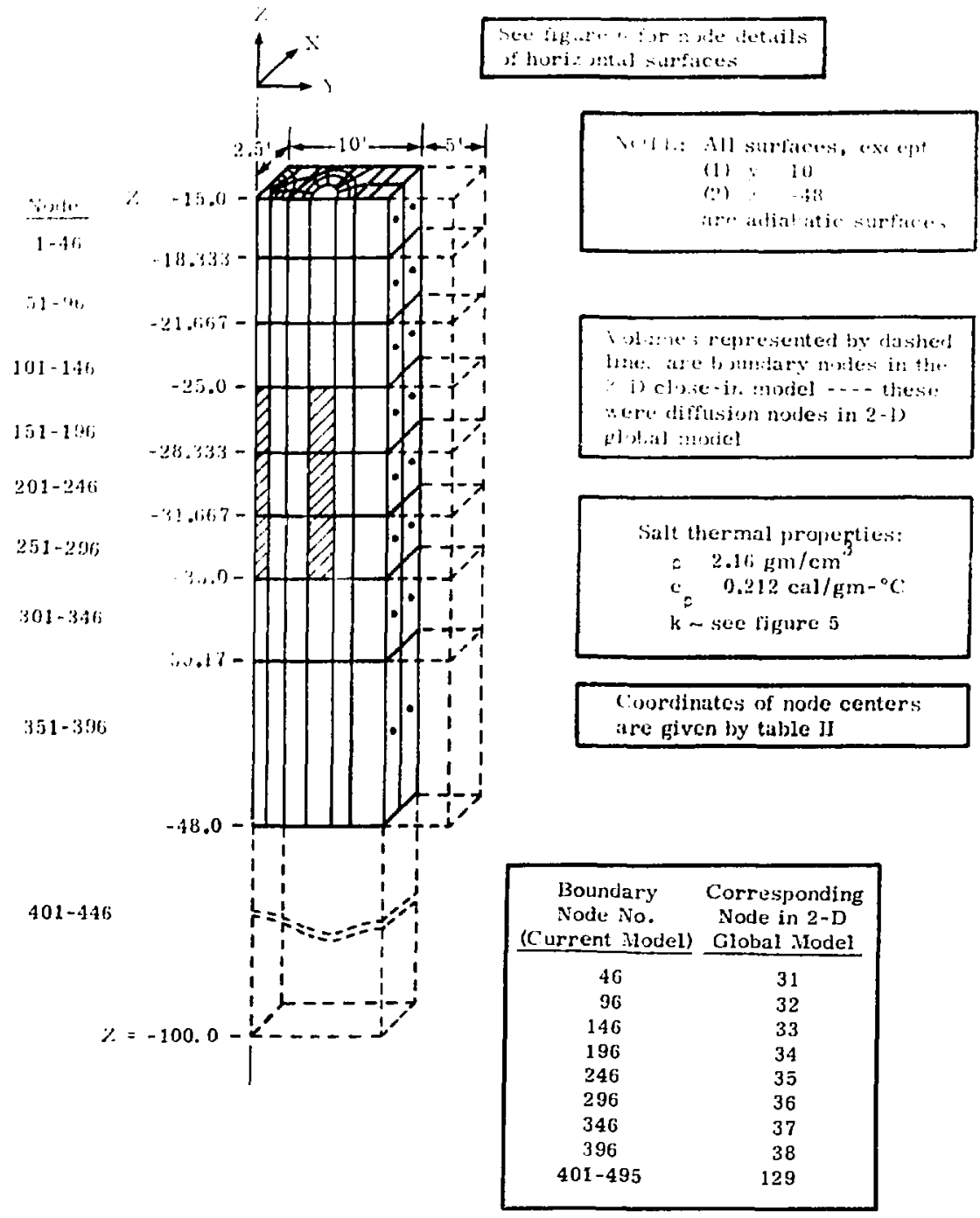

Figure 5. 3-D "Close-in" Thermal Model Geometry 
NODE NUMBERS SHOUN ARE FOR HORIZONTAL LAYER $2=-15,0$ TO $2=-18.333$ AND RANGE FRON 1-45 (PLUS DOLWDARY NODE 4E, NOT SHOLN).

MODE MUMBERS FOR OTHER HORIZONTAL LAYERS IMCREASE BY 50 PER LAYER.

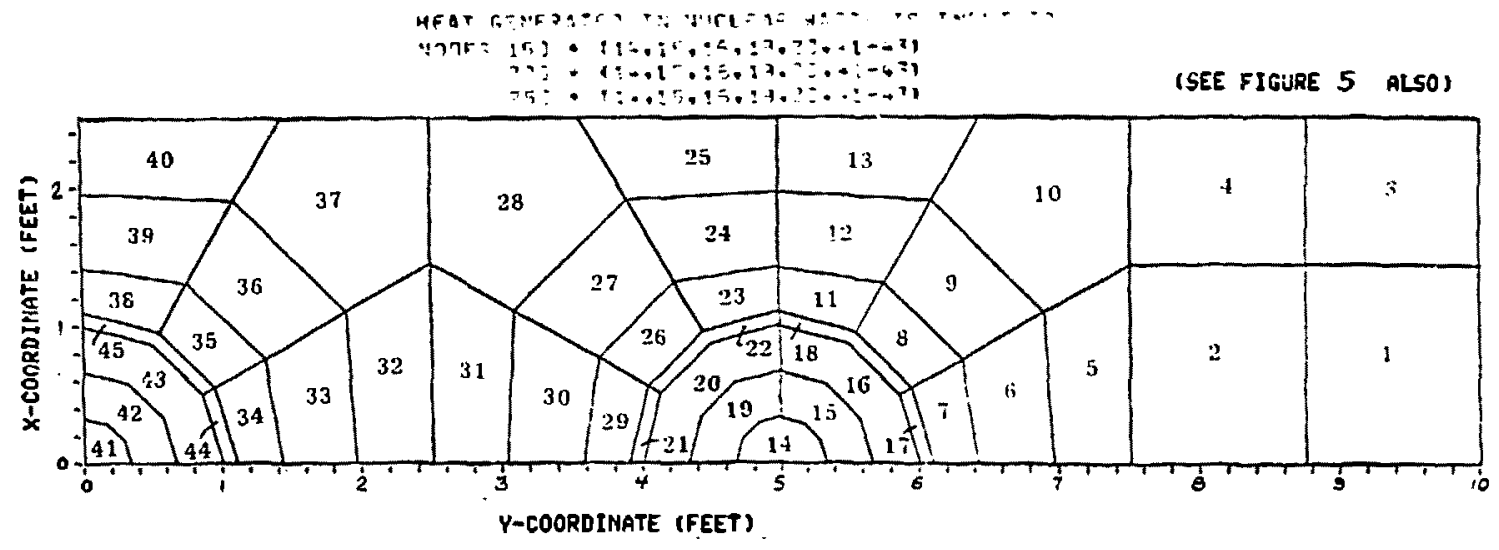

Figure 6. 3-1) Close-in Thermal Model for lob: Waste Node Numbers in l.aver 1 

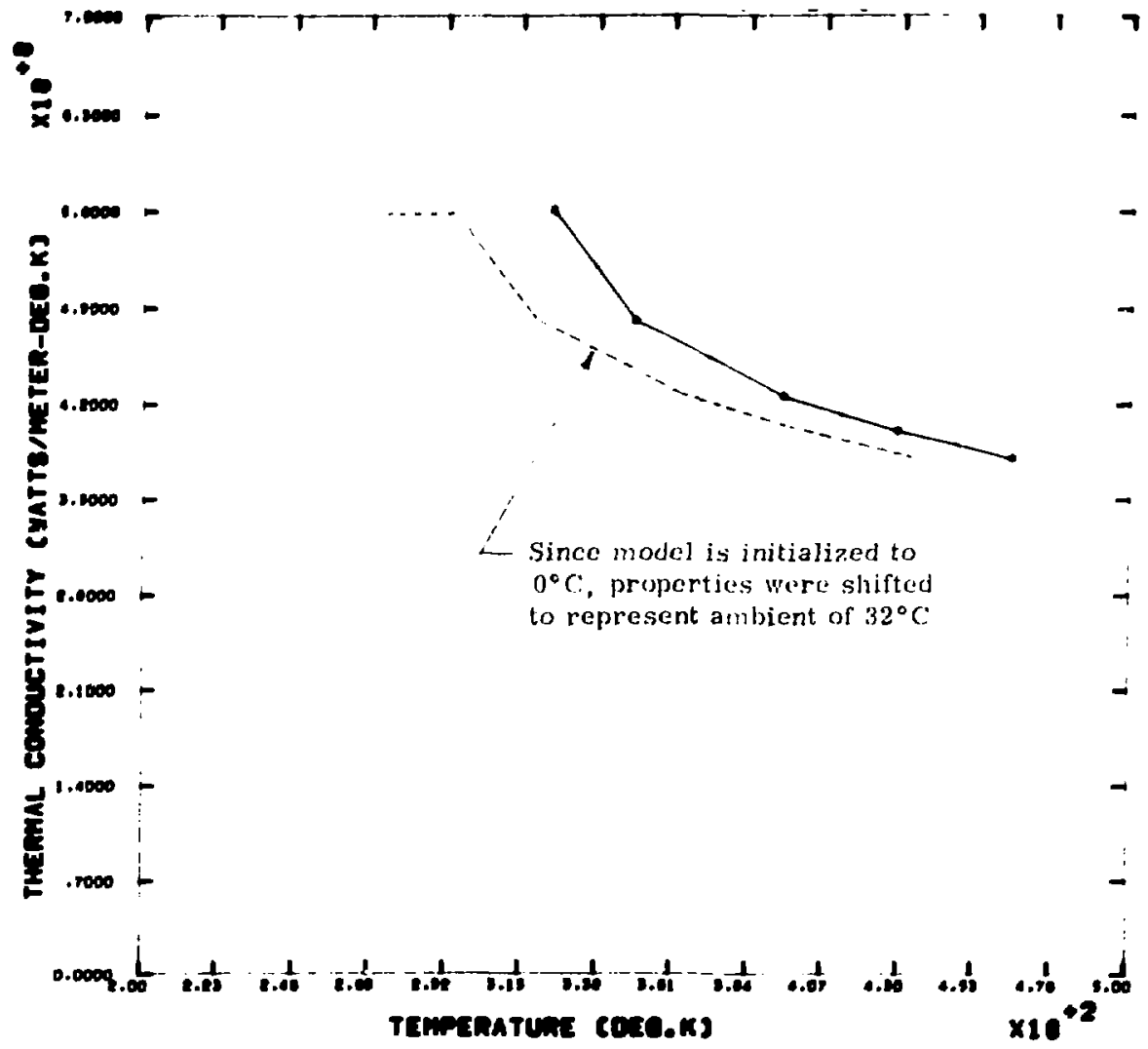

Figure 7. Thermal Conductivity of Salt 


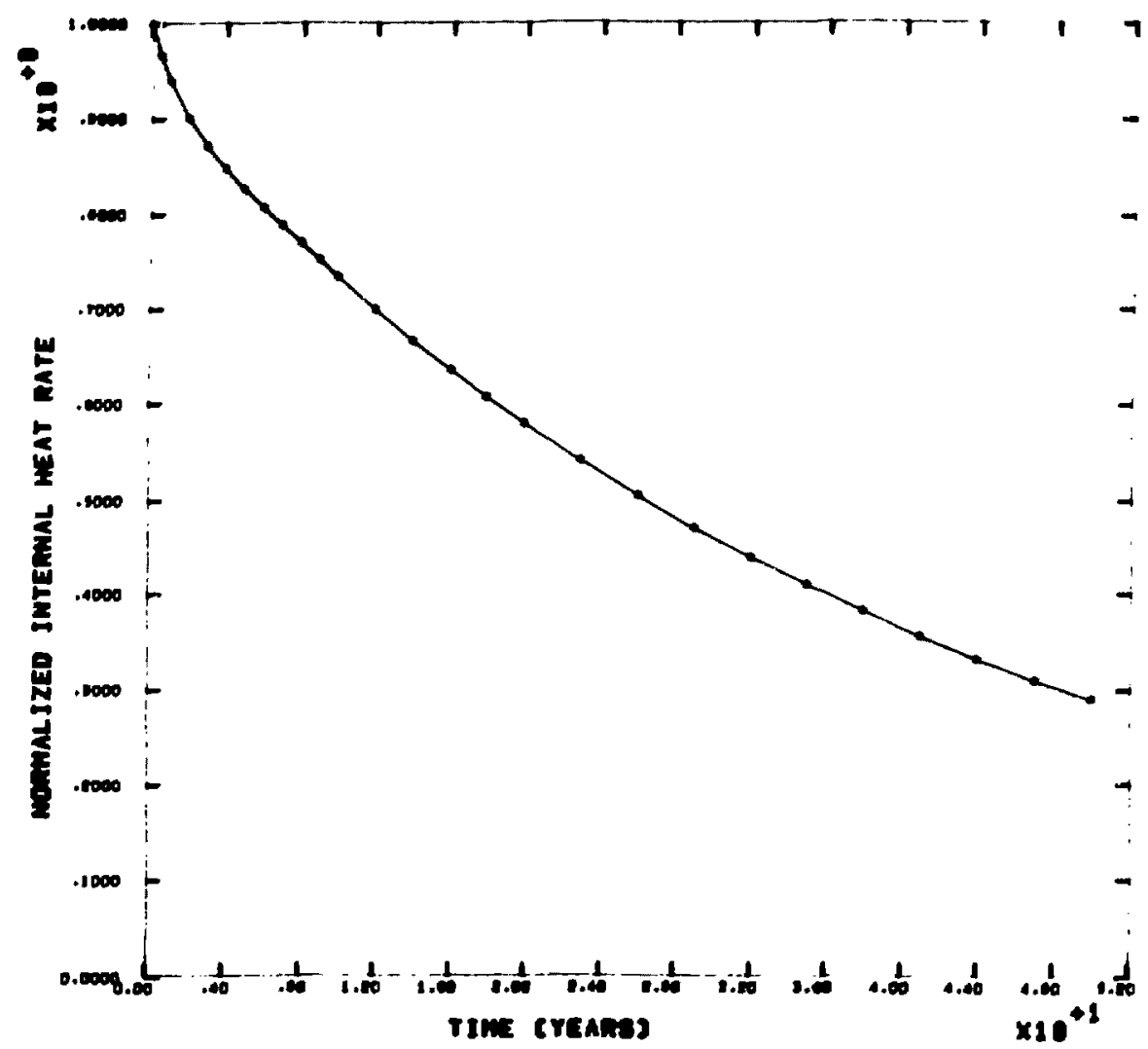

Figure 8. Normalized Heat Generated by Waste 


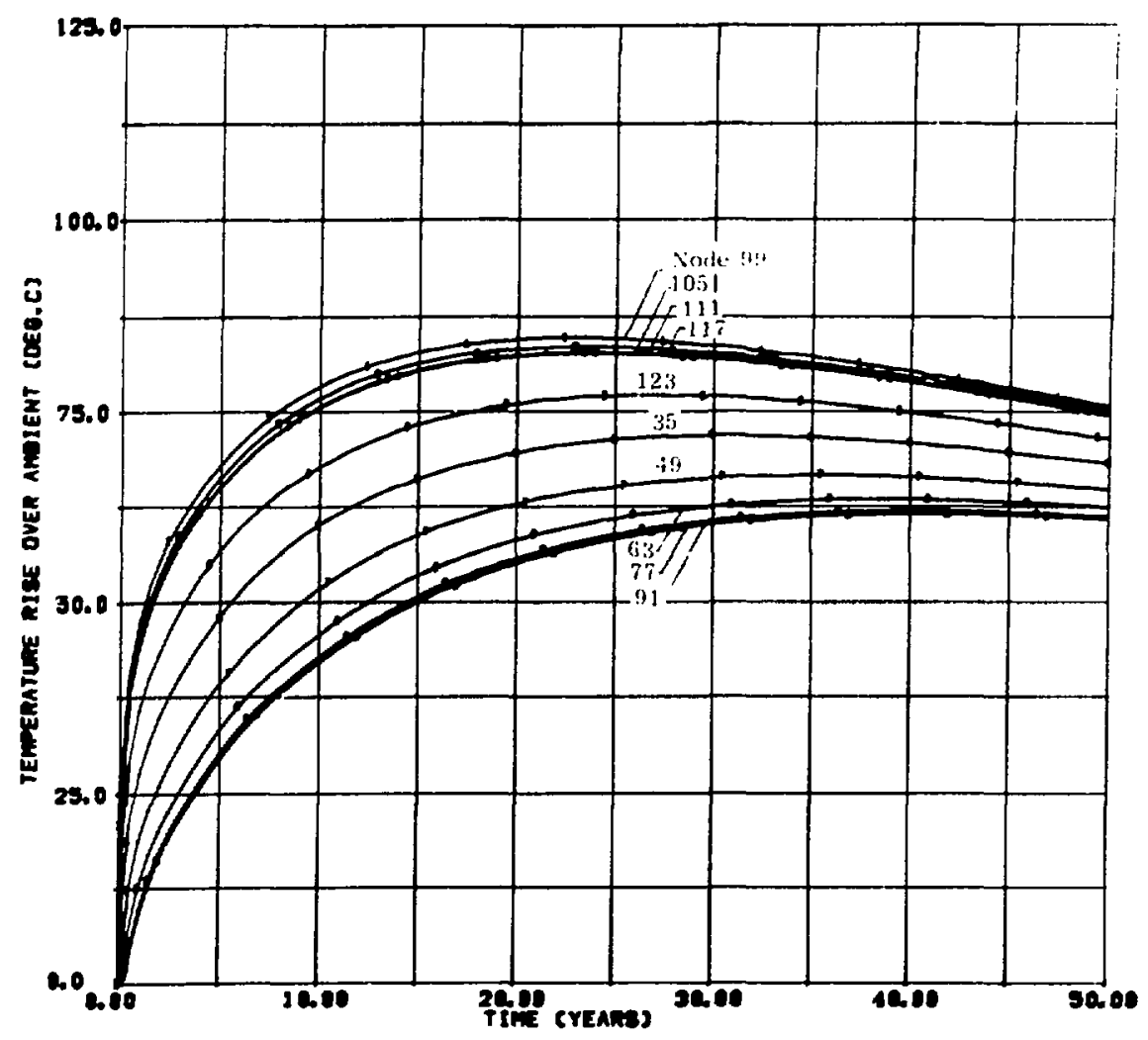

Figure 9. 2-D Salt Model for DOE Waste---Nodes Along Horizontal Path at Mid-Canister Depth 


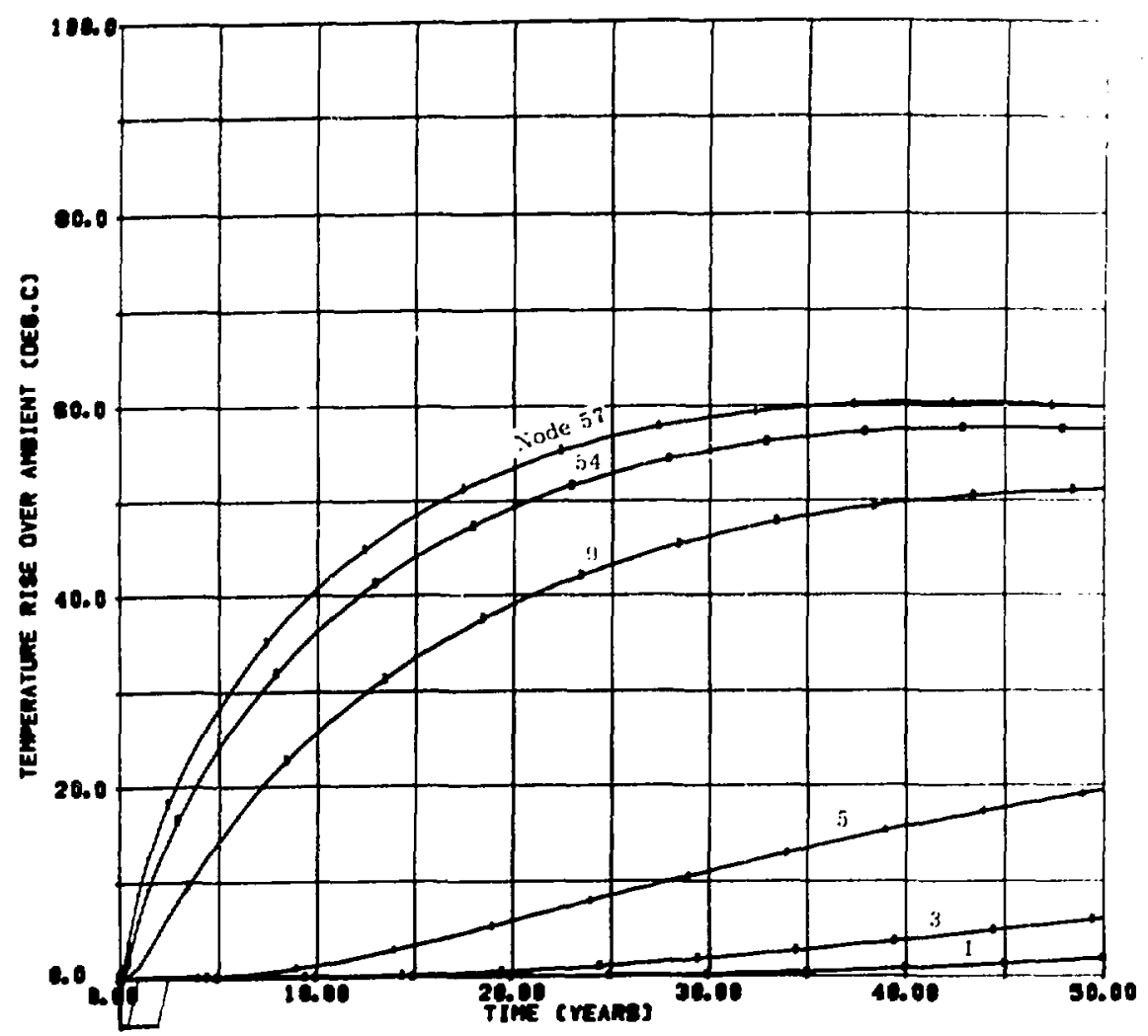

Figure 10. 2-D Salt Model for DOE Waste--Nodes Along Vertical Path Upward from Access Tunnel 


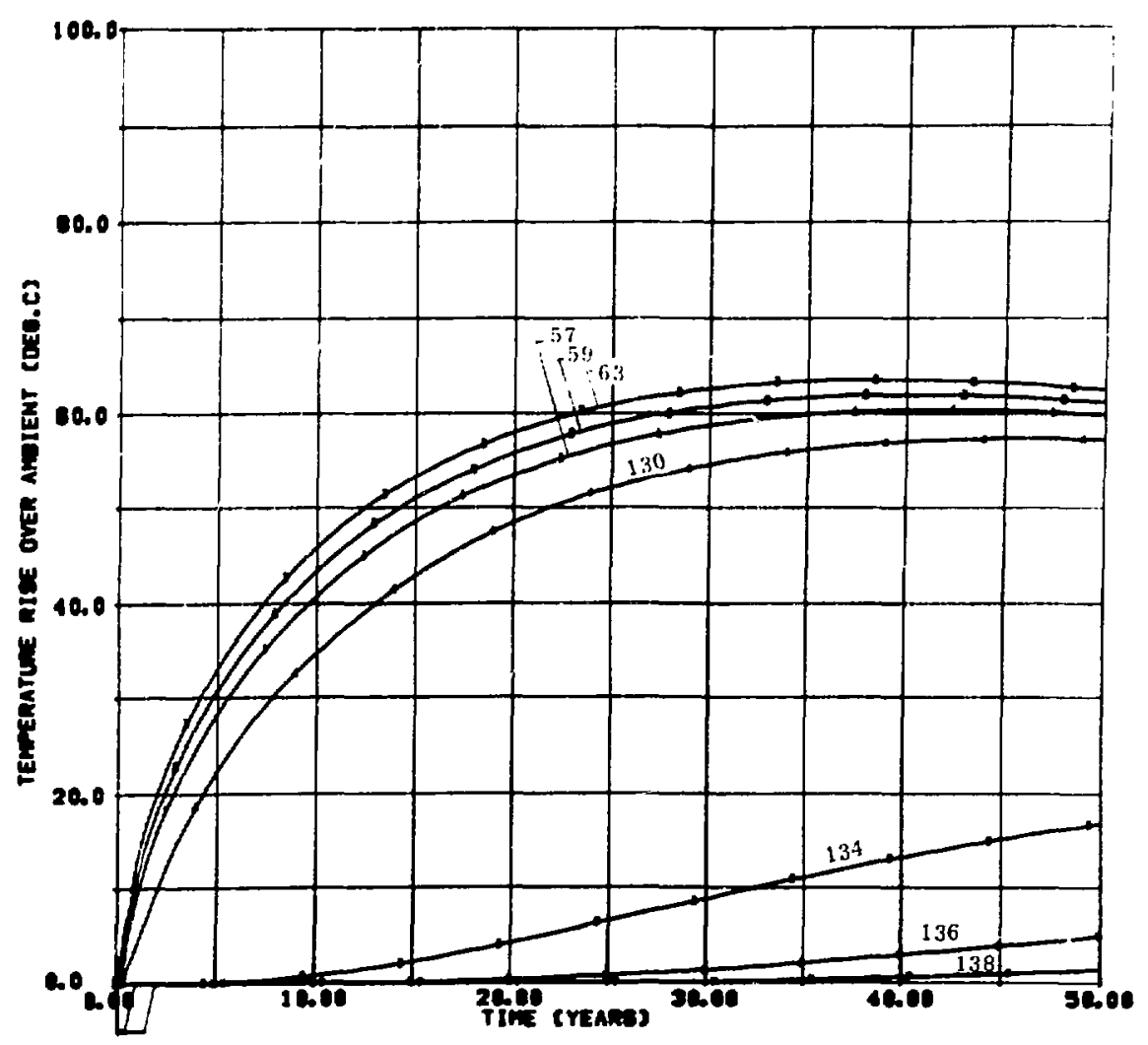

Figure 11. 2-D Salt Model for DOE Waste---Nodes Along Vertical Path Downward from Access Tunnel 


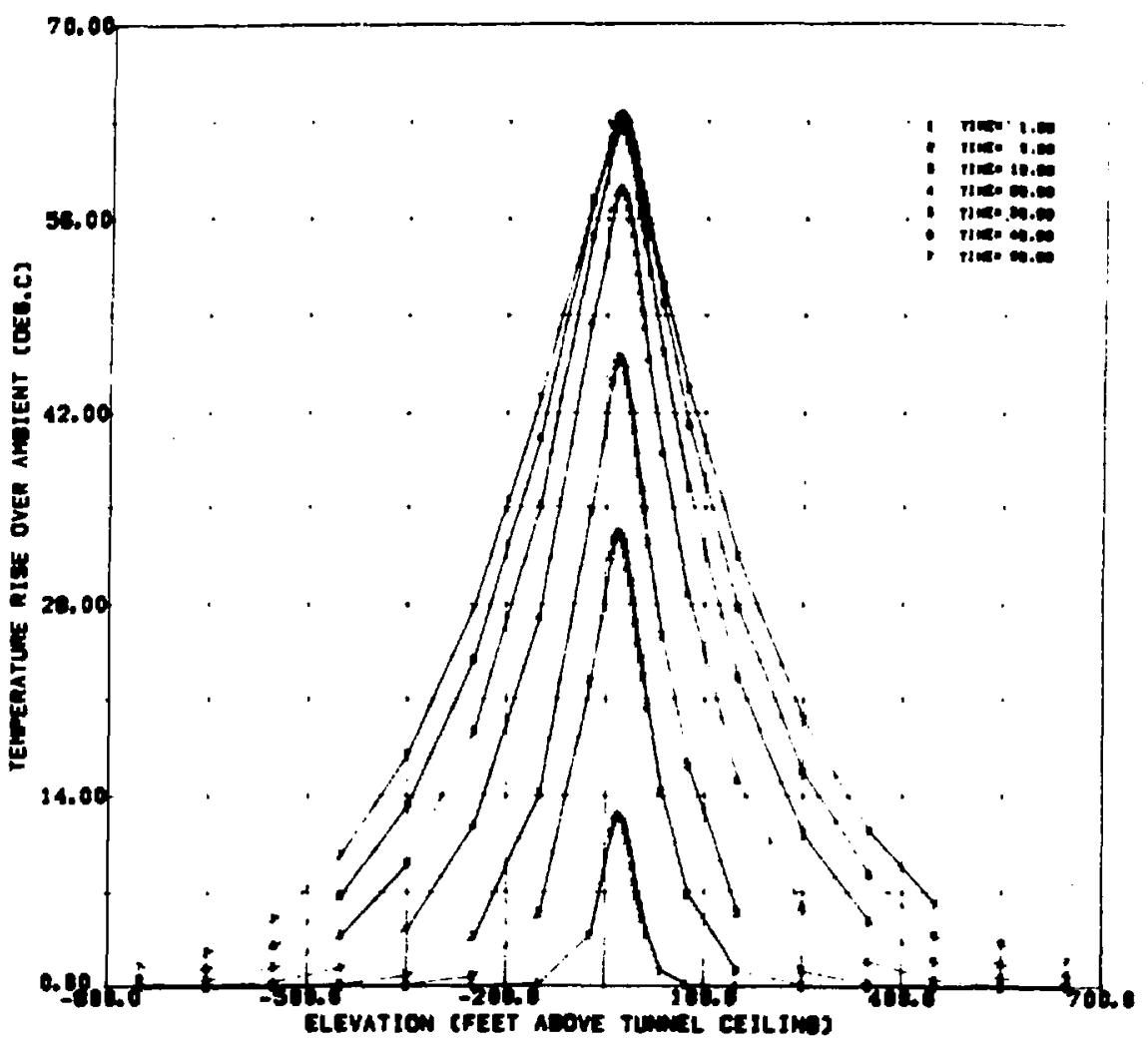

Figure 12. 2-D Salt Model for DOE Waste--Vertical Temperature Profiles 30.0 Feet off Tunnel Centerline 


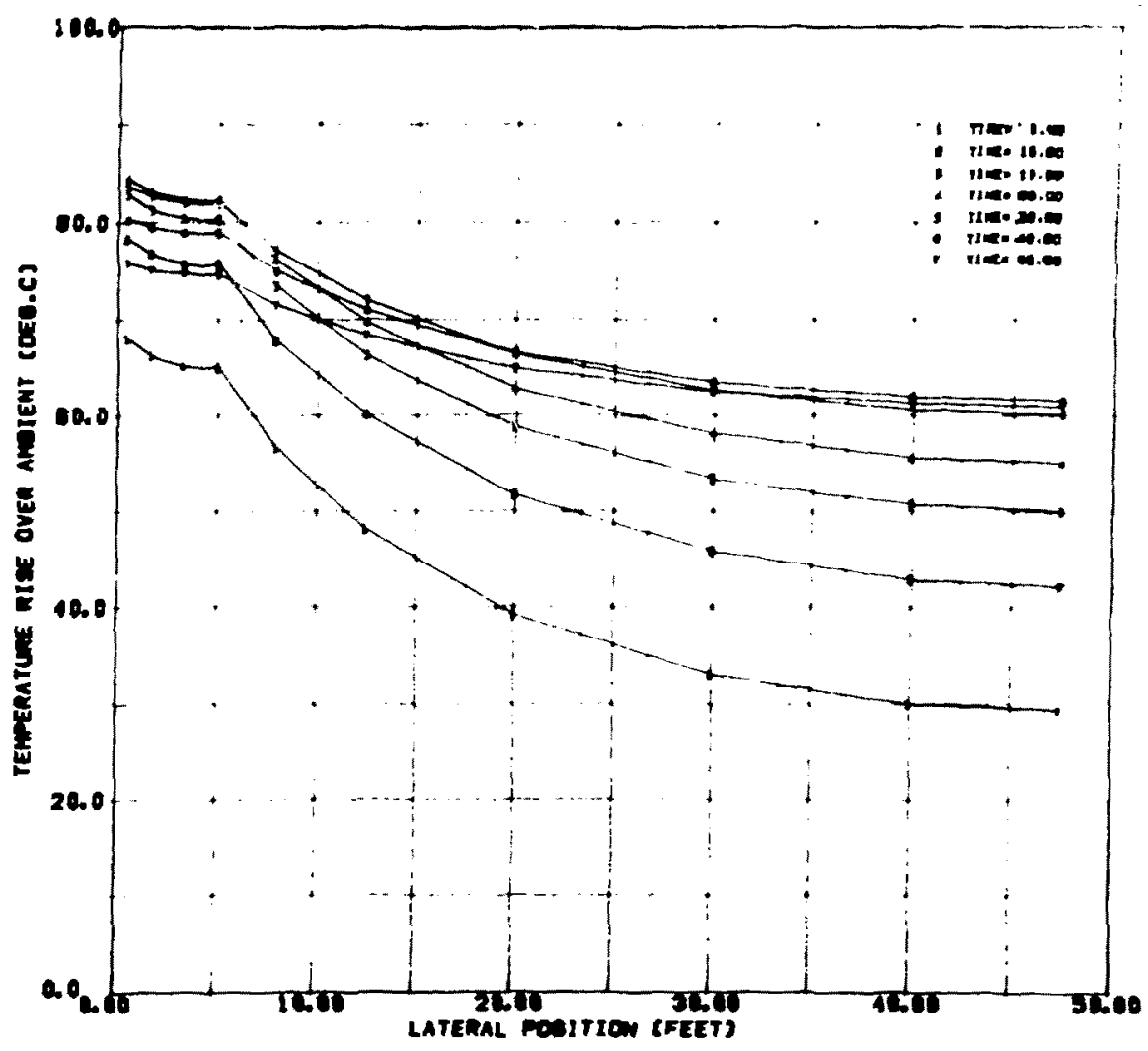

Figure 13. 2-D Salt Model for DOL Waste--Lateral Temperature Distribution at $Z=-30$ Feet 


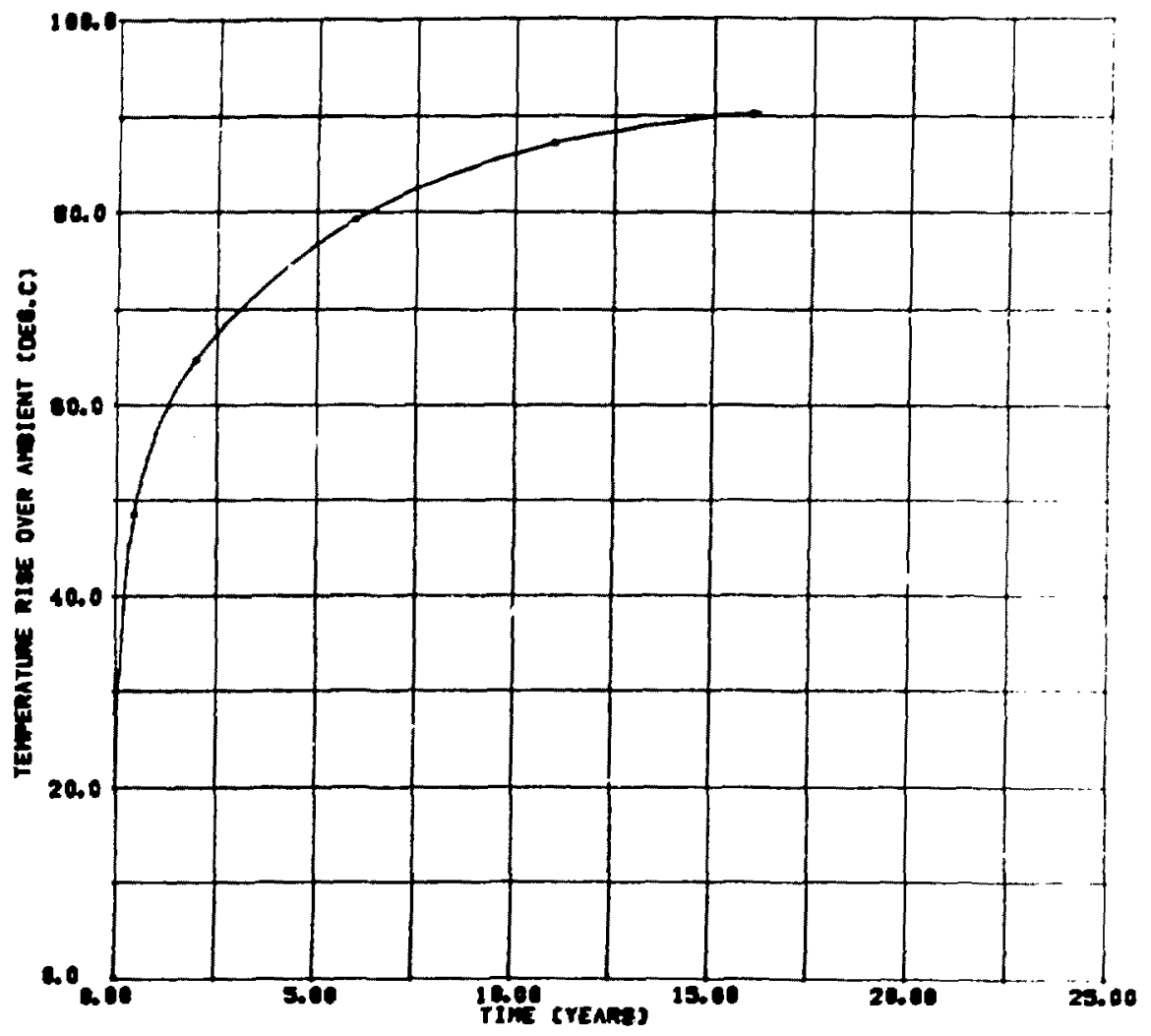

Figure 14. 3-D Close-in Salt Model for DOE Waste--Pcak Temperature in Bedded Salt 


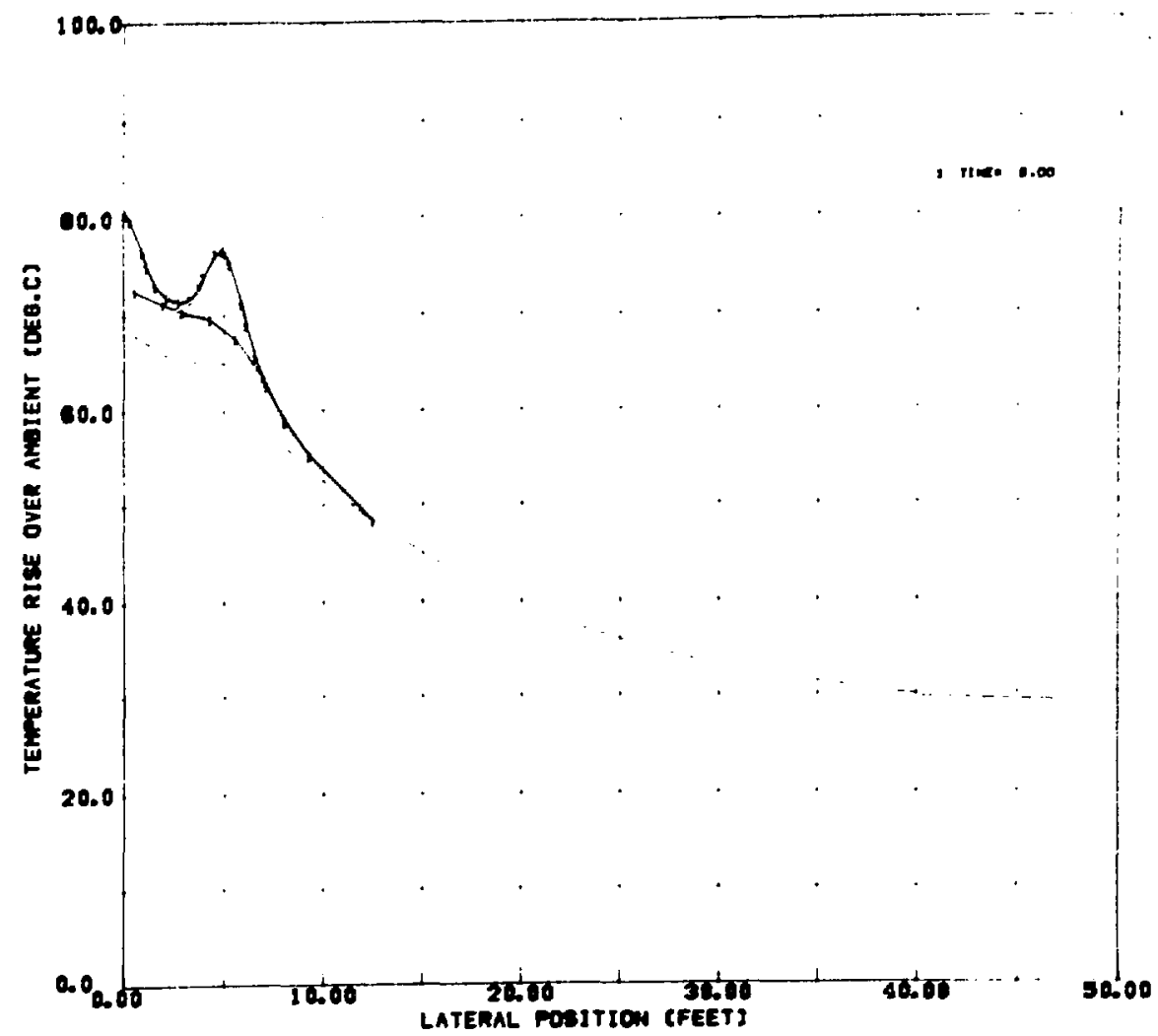

Figure 15. 3-D Close-in Model for DOE Waste--Lateral Temperature Distributions at $Z=-30$ Feet - Time $=5,00$ Years

27 


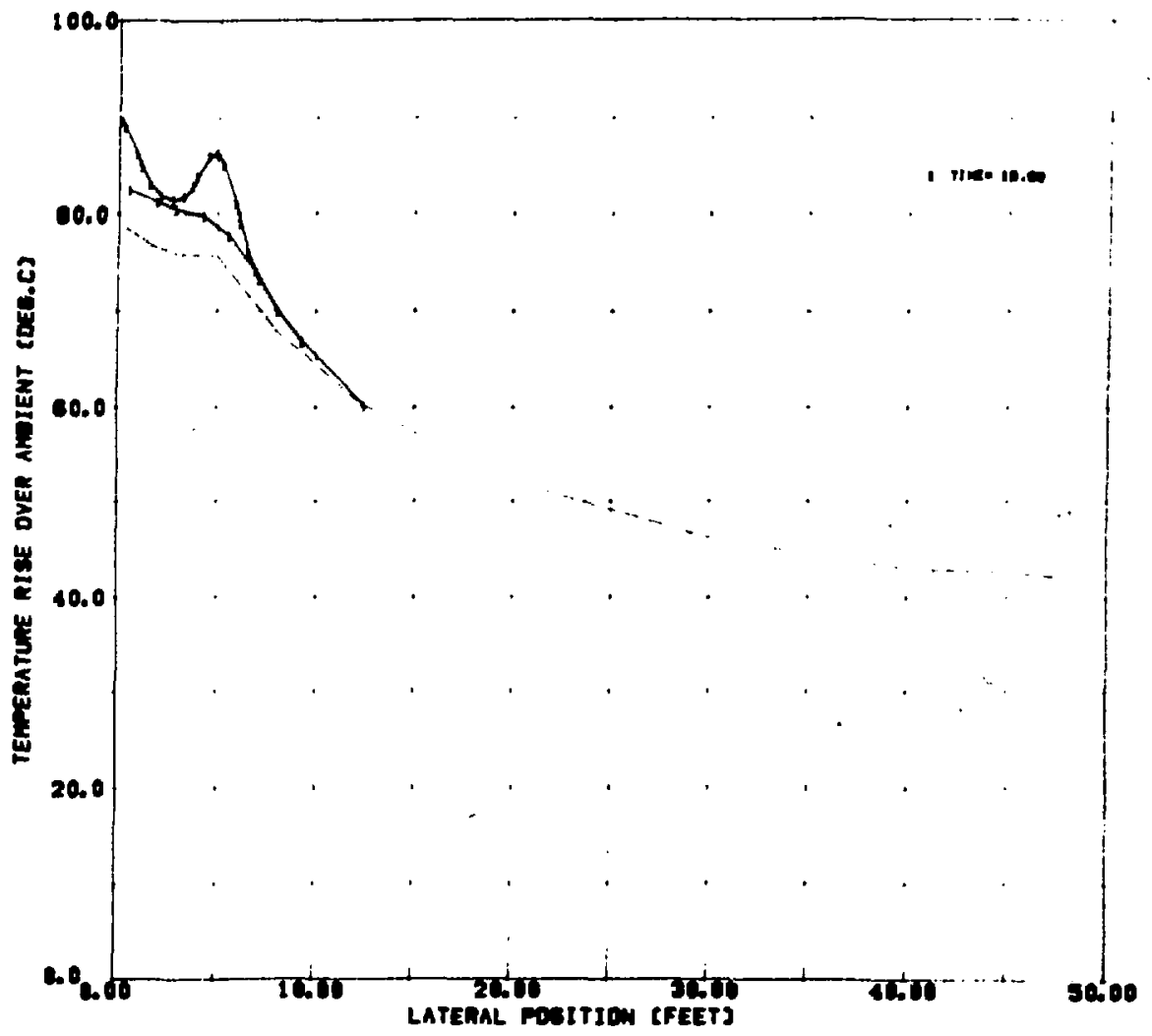

Figure 16. 3-D Close-in Model for DOE Waste--Lateral Temperature Distributions at $Z=-30$ Feet - Time $=10.00$ Years 


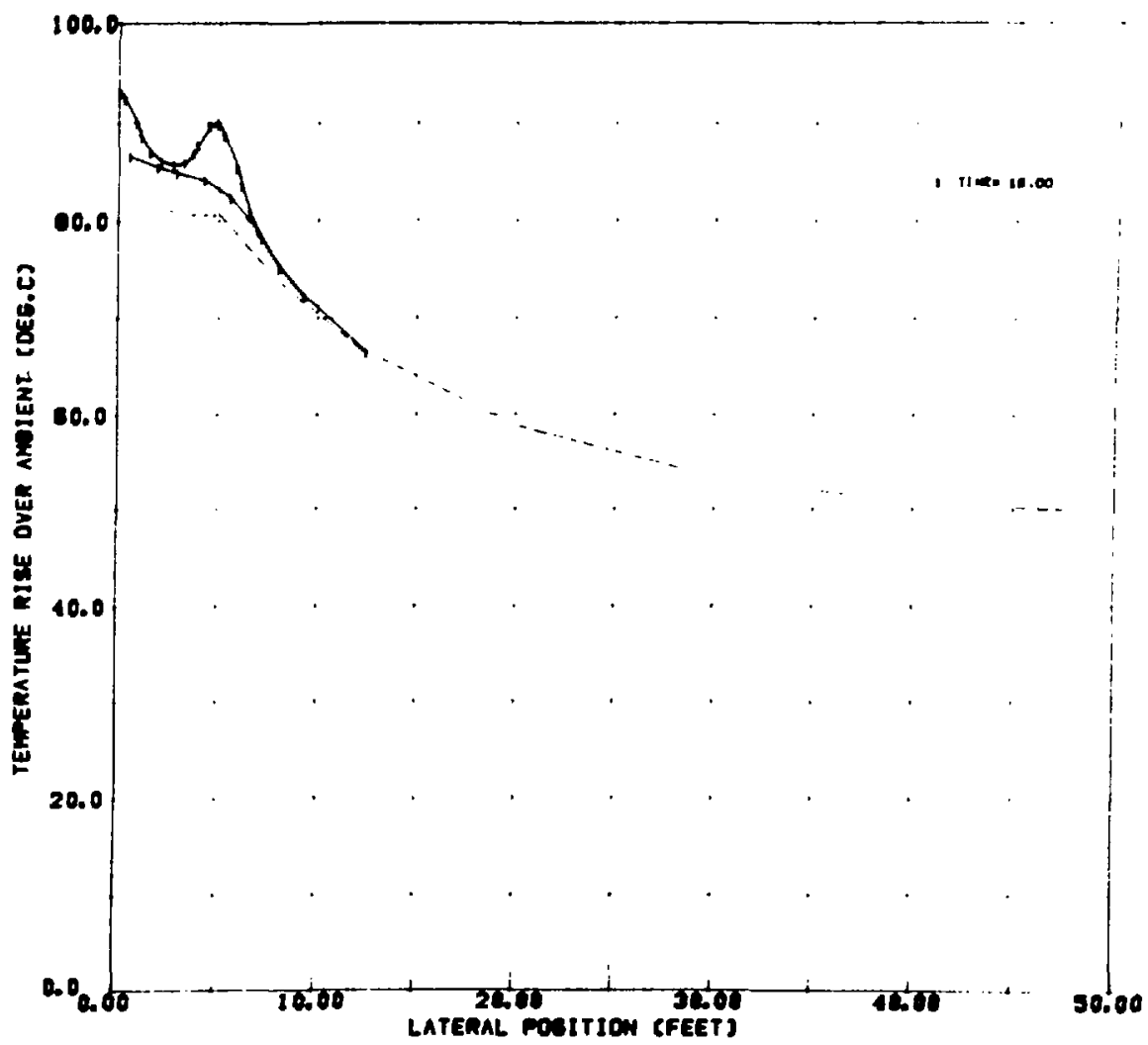

Figure 17. 3-D Close-in Model for DOE Waste--Lateral Temperature Distributions at $Z \quad-30$ Feet - Time $=15.00$ Years

29 
T.Mli1.i: !

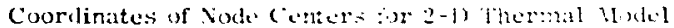

\begin{tabular}{|c|c|c|c|}
\hline $\operatorname{mox}_{\text {NO. }}$ & $x$ & $y$ & $z$ \\
\hline I & $1,25 \mathrm{CJ}$ & $x+.0: 1.1$ & ינניז,יפים \\
\hline ? & $1,350 i$ & $-5, x+30$ & 503.0500 \\
\hline j & $1,>5 \leq n$ & 70.0020 & $400.620=$ \\
\hline 4 & $1.750 \mathrm{C}$ & 25.2530 & $3=3.69 ;$ \\
\hline 5 & $1.050 ?$ & $7 r_{1}, \cos 5$ & 953.543 \\
\hline ? & $\begin{array}{l}1.2500 \\
1.7500\end{array}$ & $\begin{array}{l}75.6796 \\
95.00\end{array}$ & $\begin{array}{r}196.1310 \\
79.0930 !\end{array}$ \\
\hline . & 142500 & 5.5209 & T. Yis \\
\hline 9 & 1.2506 & 10.0030 & $53, \cdots r, 0$ \\
\hline 10 & $1.250 \mathrm{u}$ & $\therefore \times 5: 0$ & 17.453 \\
\hline 11 & 1.7500 & $r .0003$ & $12.0=50$ \\
\hline 12 & $1,25,0 e$ & $17.50 \%$ & $\therefore$ a c a $50^{\circ}$ \\
\hline is & 1.7500 & 25,0000 & $17.945 ?$ \\
\hline 14 & 1.7500 & 4207300 & $12 \cdot c+50$ \\
\hline is & 1.756 & $.45,50$ & 5,4067 \\
\hline 16 & $1,75 i:$ & $.650 \mathrm{~J}$ & $1.6,75$ \\
\hline it & 1.750 & 1.7600 & $5,4163^{\circ}$ \\
\hline is & 1.25rt & 1.7000 & 1.075 \\
\hline 10 & $1.75 \mathrm{AB}$ & T.ses & [. Wif? \\
\hline 20 & 1.7500 & 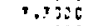 & I. hing? \\
\hline 31 & 1.7549 & G.8:0 & 5.6149 \\
\hline 22 & 1.2500 & 5.6तid & $\therefore$ सhat \\
\hline 11 & 1. $\rightarrow$ GC? & $7.750 \mathrm{~J}$ & $c .4 ! 67$ \\
\hline 36 & $1, .553$ & Tatiojo & iobsior \\
\hline 75 & 1.2502 & $15,5 \geq 00$ & $=.415^{\circ}$ \\
\hline $2 i$ & 1.3566 & 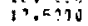 & I.tah? \\
\hline$?$ & fosect & $1,, 5010$ & -1. KSB? \\
\hline in & i.price & $12,5,020$ & $-x_{0}+1 F$ \\
\hline 27 & 1.2509 & $1 \rightarrow 0,500$ & $-7,408$ \\
\hline 30 & 1.950 .1 & $\because$ Sojo & $-13,143$ \\
\hline 11 & $1,750 \%$ & $12+1830$ & $-16+445^{\circ}$ \\
\hline 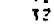 & 1.2500 & $i>1,1 \geq 0$ & -23.035 \\
\hline 13 & $1.250:$ & $12,50: 0$ & $-2 \times 140$ \\
\hline is & 1.7590 & 17.5030 & $-75,6567$ \\
\hline 35 & 1.2560 & $1, ;, x, 3=0$ & $-3 r \cdot[\div 30$ \\
\hline in & 1.7527 & $15,+, \pi 53$ & $-7,1+13$ \\
\hline 37 & 1.9592 & 13,504 ? & $A 7.64=1$ \\
\hline in & $1.25=8$ & 17,5300 & -21.1330 \\
\hline 19 & 1.904 & ? נ. & $5.41 \%$ \\
\hline 40 & 1.2500 & 20,0020 & 1, litis, \\
\hline $4 i$ & 1.7 .503 & 20.6303 & $=1$. inat \\
\hline 4? & $1,75,00$ & 01303 & -5, ot: \\
\hline 4x & 1.2590 & $73.0+13$ & $-9 . r, 3$ \\
\hline 44 & 1,2534 & $3=0 \leqslant 01$ & -1 I. $x<<$ \\
\hline 45 & 1.2501 & ב נ0.0. & $-: 5, k \leq x, 7$ \\
\hline 45 & 1.2500 & ig. & $\because 20.0360$ \\
\hline 47 & 1,3500 & 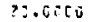 & $-7 \times, 9133$ \\
\hline 6. & 1.2500 & ذ23.0 & -7moghtit \\
\hline 69 & 1,2502 & $20.00 \div 0$ & - ניt \\
\hline 50 & 1.7507 & טנחה. סי & $-17,7,18$ \\
\hline
\end{tabular}
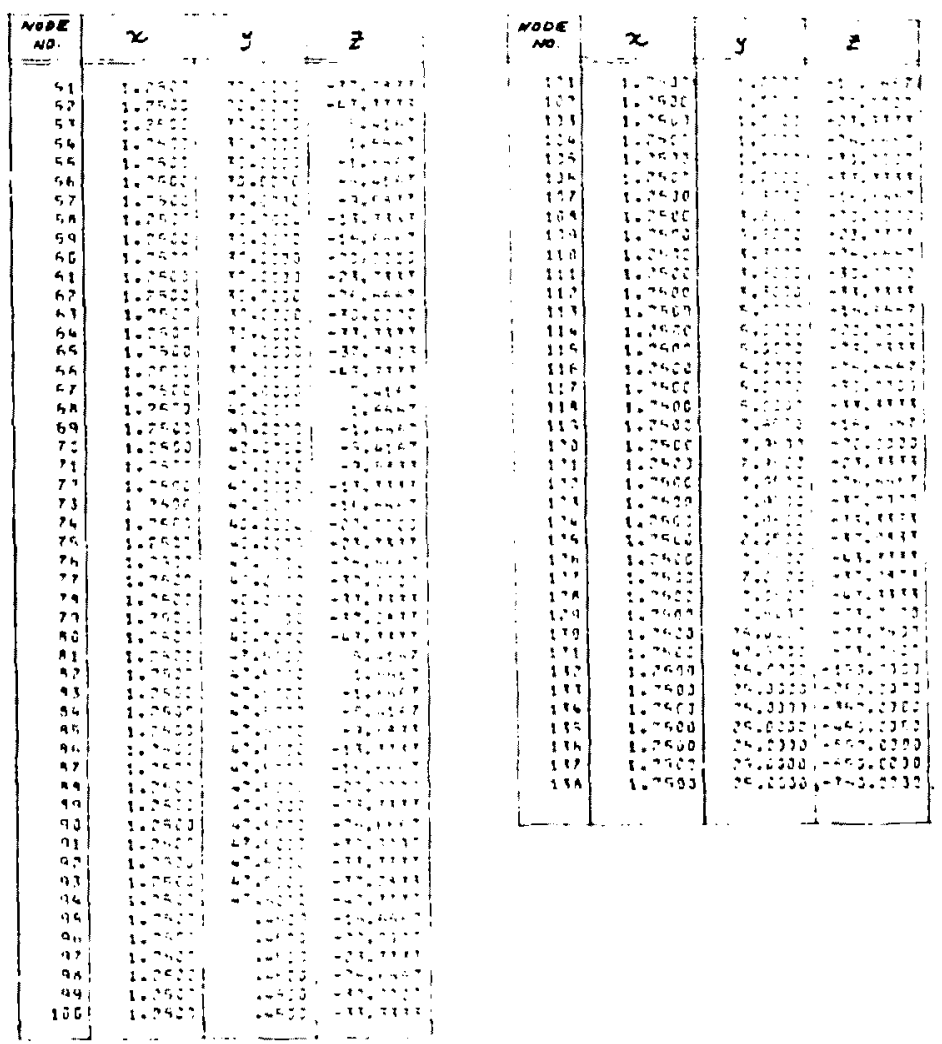
T.MISIE II

Coordinates of Node Centers for $3-1$ Thermal Nodel

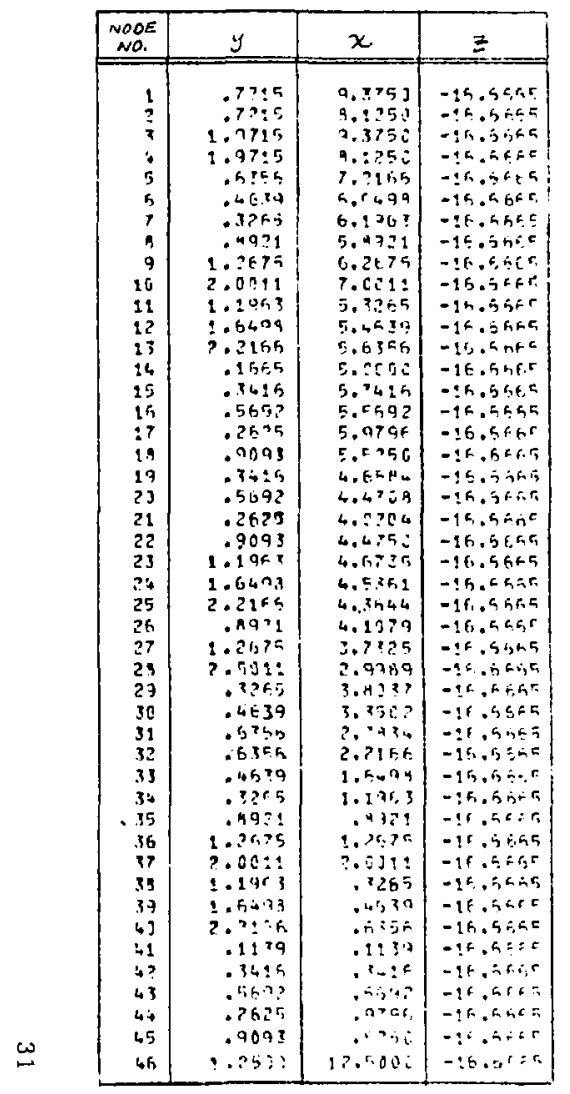

TH= FM! L

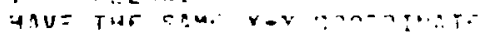

Lะ

$$
\begin{aligned}
& 1-5: \\
& \because 1-: \dot{c} \\
& : \equiv 1-13 f \\
& 71 \text { - } 72: \\
& \text { ग5 - T5 } \\
& 3 ? 1-34 \\
& \text { T:- } \quad \therefore 6 \\
& +11=+-5
\end{aligned}
$$

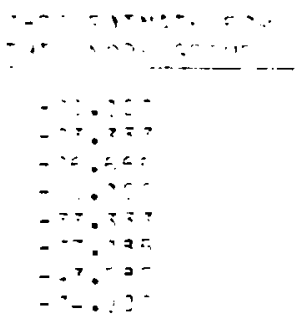




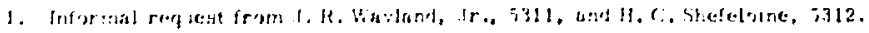

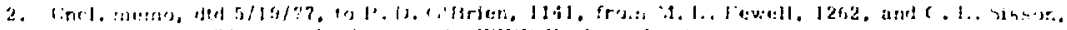

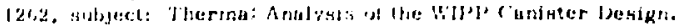

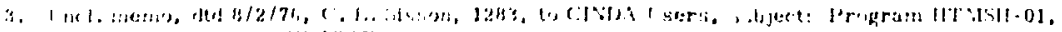

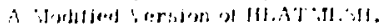

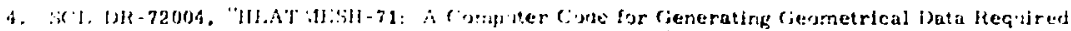

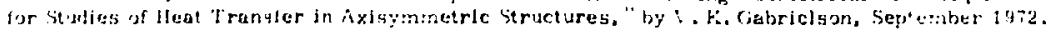

5. I ה-A Cinerituen computers," coctiber 20. 1957.

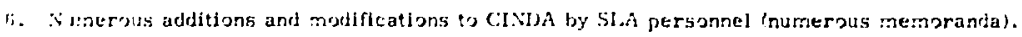

7. Ji-31'-1,7-2H7, 2p. cit. 


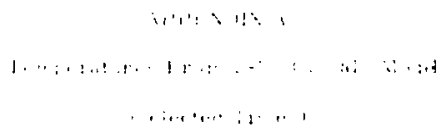

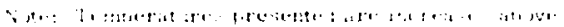

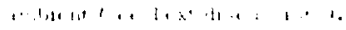




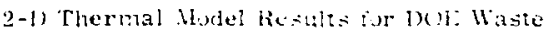

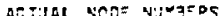

\begin{tabular}{|c|c|c|c|c|c|}
\hline \multirow{2}{*}{ 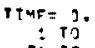 } & \multicolumn{4}{|c|}{ YFA } & \\
\hline & 20 & 0.3 & $c .0$ & 0.0 & $n+E$ \\
\hline$\because: 87$ & 40 & $0 . \Sigma$ & $i .5$ & $i, 2$ & \\
\hline 6: :7 & 50 & c.J & [. . & $\overline{0}, \mathrm{C}$ & r. 2 \\
\hline $5: 11$ & 1 & c.: & $i * i$ & $\therefore 2$ & $\because \bar{z}$ \\
\hline$\because: 7$ & 13 & 0.9 & i. & 0.5 & \\
\hline $1=1$ : & $1 ? 3$ & 2.3 & $\therefore 2$ & $0 .:$ & $\therefore$ \\
\hline $17: 7$ & 11 & E. . & : : & $c .2$ & \\
\hline
\end{tabular}

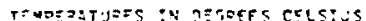

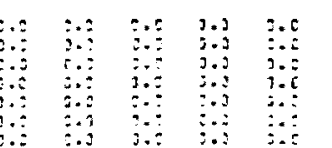

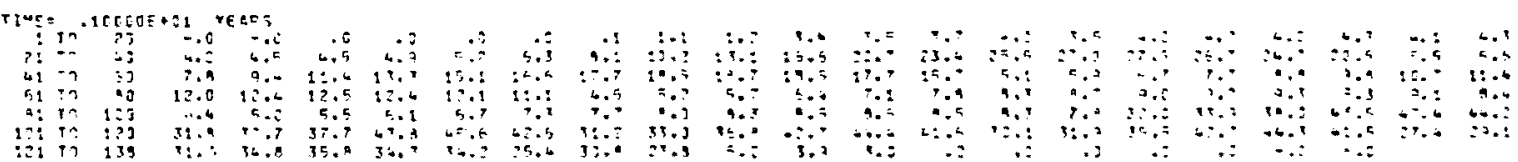

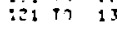

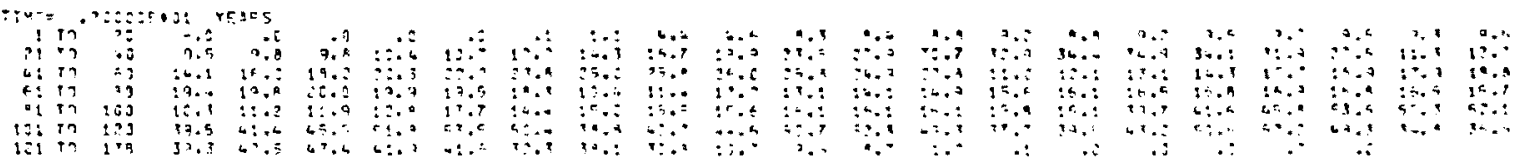

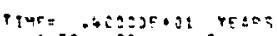

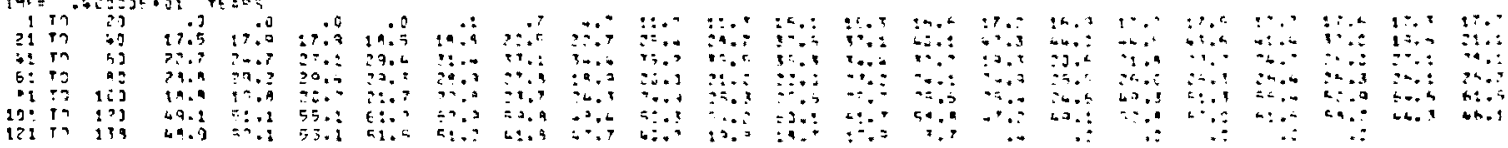

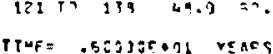

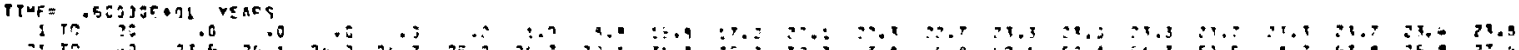

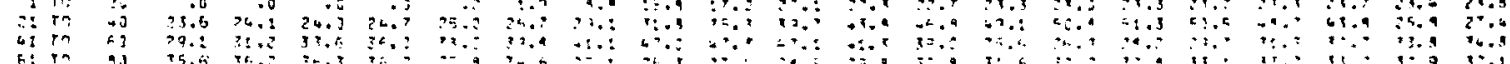

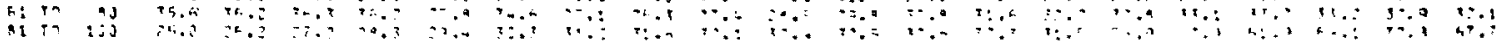

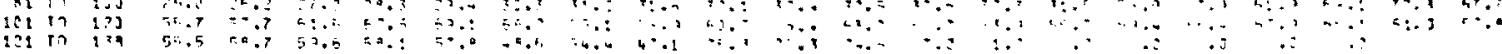


2-1) Thermal Model Result ior b()t: Mante

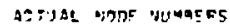

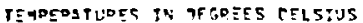

\begin{tabular}{|c|c|c|c|c|c|c|c|c|c|c|c|c|c|c|c|c|c|c|c|c|c|c|}
\hline 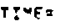 & & 37 & & & & & & & & & & & & & & & & & & & & \\
\hline 1 & & 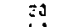 & .3 & .0 & $=C$ & .1 & .6 & T.6 & $2=97$ & $3:-7$ & 22.7 & 27.1 & 27.2 & 57.5 & 29.9 & $28=0$ & 29.3 & 28.6 & $29-1$ & $\because-?$ & 39.6 & 230 \\
\hline 21 & T? & 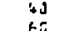 & 29.6 & 27.1 & 29.0 & $20, ?$ & 13.0 & 31.5 & $x+2$ & $7 \doteqdot .7$ & +7.6 & $6 \div ;$ & 67.3 & $5:-9$ & 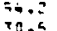 & 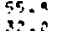 & $=5.2$ & 55.4 & $53 \cdot 3$ & 49.7 & $3: 3$ & $3: .5$ \\
\hline $\begin{array}{l}41 \\
51\end{array}$ & T? & A. & $\begin{array}{r}6.2 \\
60.3\end{array}$ & $\begin{array}{l}36.3 \\
41.3\end{array}$ & $\begin{array}{l}38.8 \\
41.6\end{array}$ & $\begin{array}{r}4: 2 \\
64.5\end{array}$ & $\begin{array}{l}47.3 \\
49.1\end{array}$ & $\begin{array}{l}55.3 \\
30.9\end{array}$ & $\begin{array}{l}4.4 \\
3 \pm .2\end{array}$ & $\begin{array}{l}47, ? \\
31.6\end{array}$ & 32.5 & $\begin{array}{l}67.5 \\
33.7\end{array}$ & $3=2$. & $\begin{array}{ll}m, 3 \\
3,5\end{array}$ & 85.5 & 37.5 & 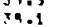 & 39.6 & 34.4 & $x=.5$ & $3 \because 3$ & 3 \\
\hline 31 & T? & :33 & 73.1 & 79.3 & 32,3 & $3 ?, 5$ & $8 . .5$ & 35.5 & 35, & $3=.7$ & 37.6 & 37.7 & כ. כינ & $z=\ldots$ & 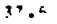 & 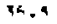 & EC.. & R.s & $5 k .5$ & 3.5 & $+c_{.2}$ & $\because \because 2$ \\
\hline$: 31$ & т T & 120 & h.C.5 & $5 ? .6$ & fh.? & 71.9 & $7 \times .5$ & $7 \because .5$ & $\because 0.7$ & 4.9 & $c=., 6$ & 75.0 & & 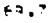 & $=9$. & $\because .5$ & $6 \rightarrow .1$ & 0.9 & $\because \geq !$ & $6=.5$ & $c_{2}=3$ & 0.7 .7 \\
\hline & & :?4 & 66.5 & 7.4 & 64.3 & 8.9 & B2. .f & $5 \times .7$ & 50.1 & $\therefore \therefore$ & $3:=5$ & 325 & $=0.7$ & $: 2.7$ & $\therefore 1$ & 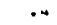 & .1 & ה . & .2 & .2 & & \\
\hline
\end{tabular}

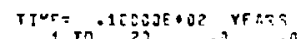

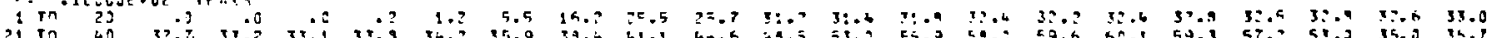

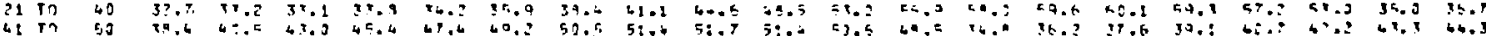

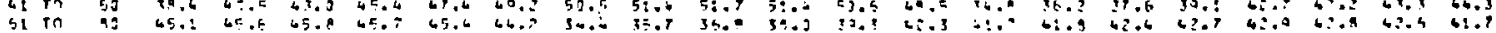

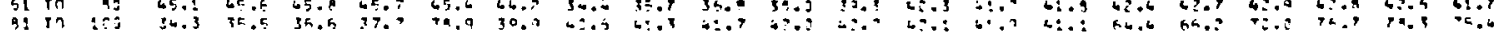

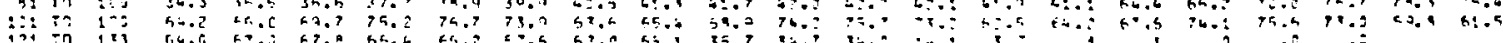

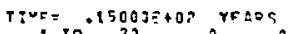

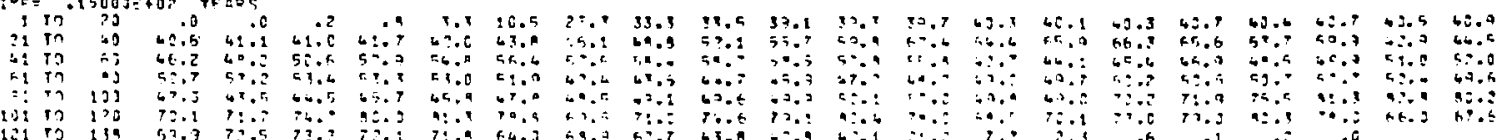

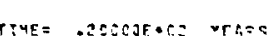

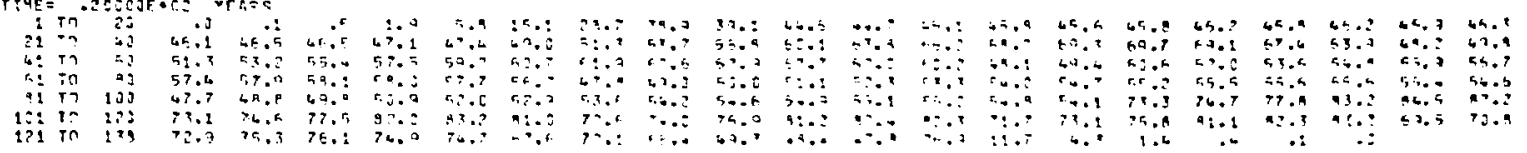

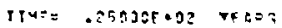

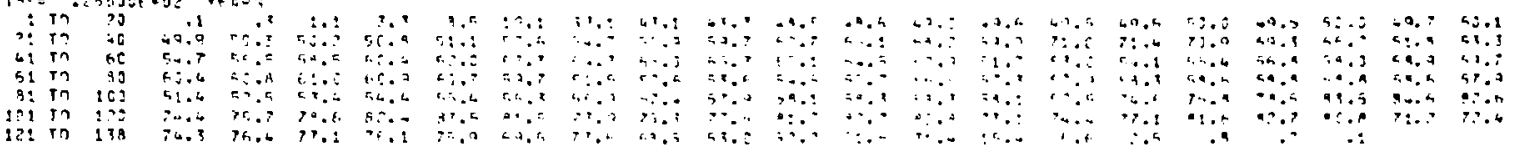




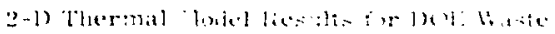

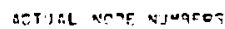

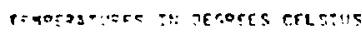

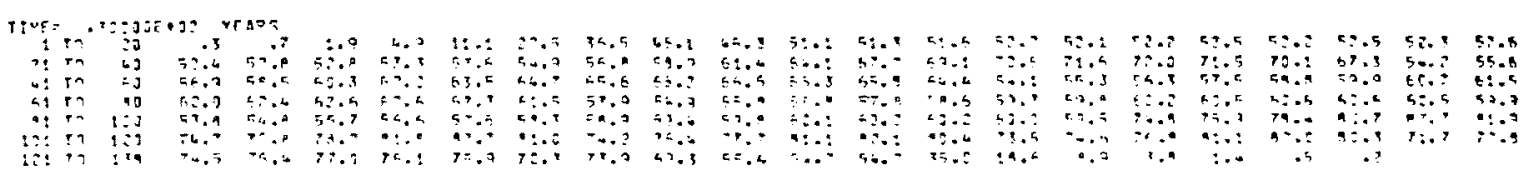

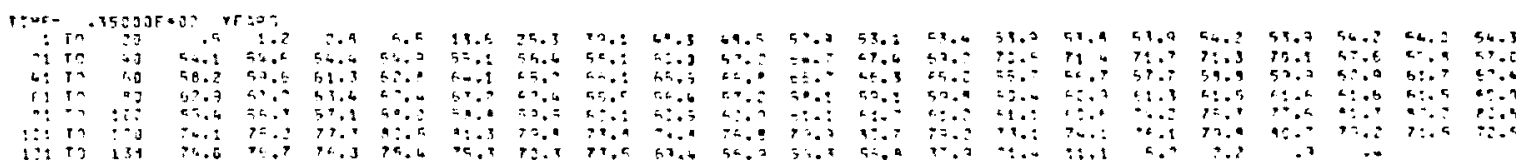

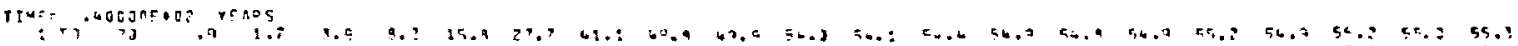

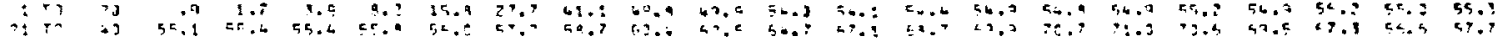

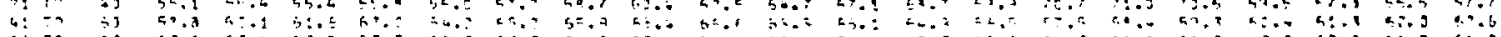

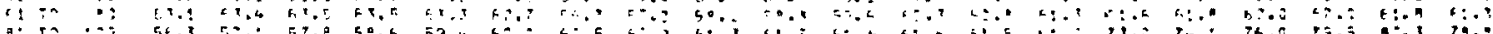

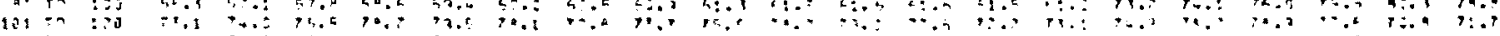

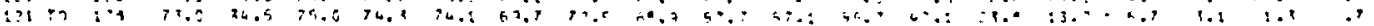

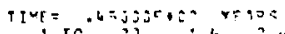

1 Ta

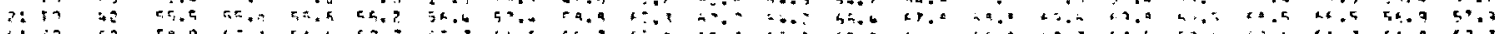

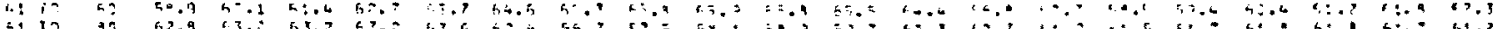

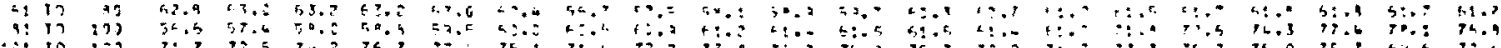

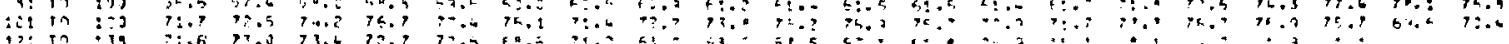
tinti=

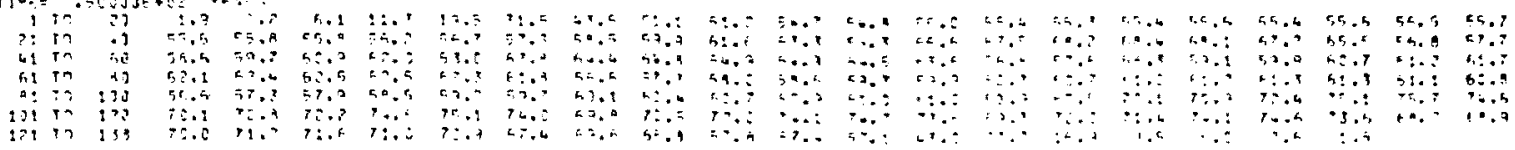




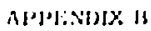

Temperiture vertus fiersedinate l.ocation

2-1) "(i]'but" Mrele]

(Yorjes Near Drtft)

This Mipendix presents a detibiled tabulation of temperatures versus coordiniate location of nole centers for all noxtes in the range $-35<z<7.5$ feet. Temperatures shrms are increases above asnbient.

Table b-1 lists the node numbers.

Fulue $11-2,013-11$ list the corresponding nole temperatures. 
THBH.t:B-1

Node Numbers versus i -7. (Cortinate

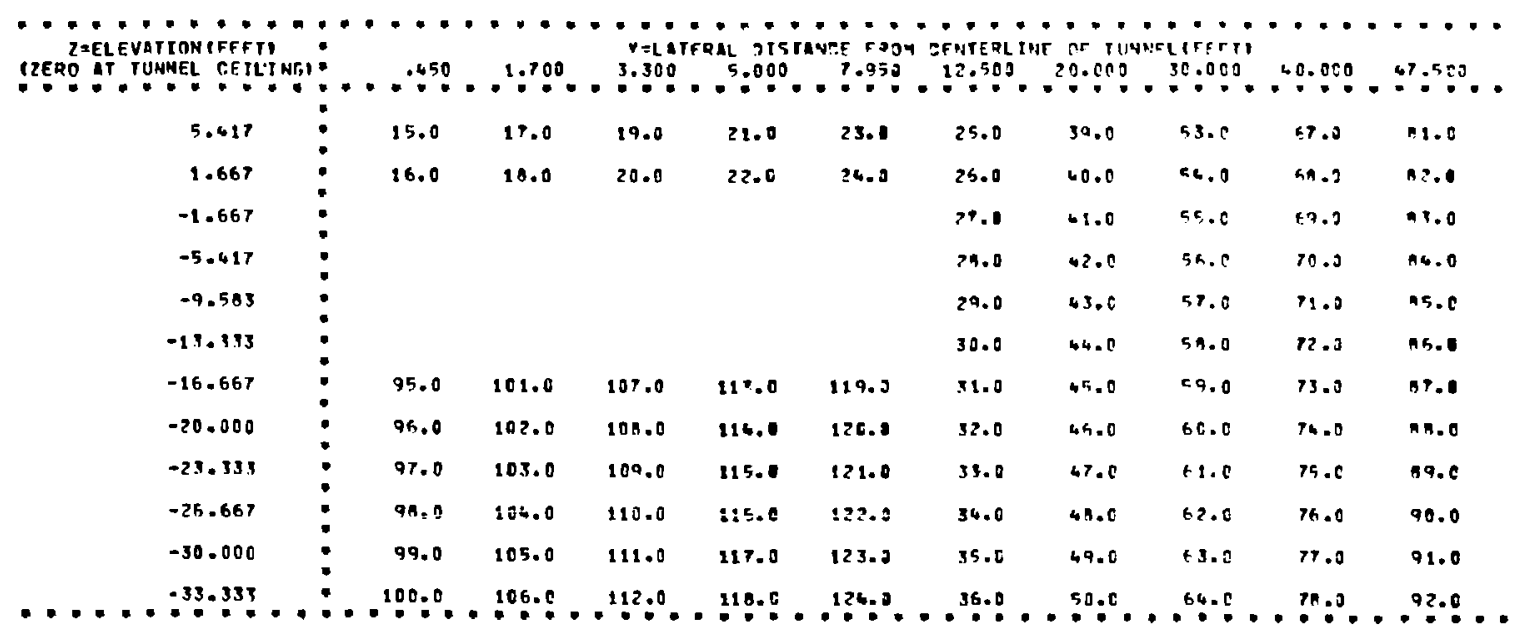


TAlBI: B-2

remperature lise dbove Ainbient cersus Y-r. Courdinates

Time (yrs) 1.0

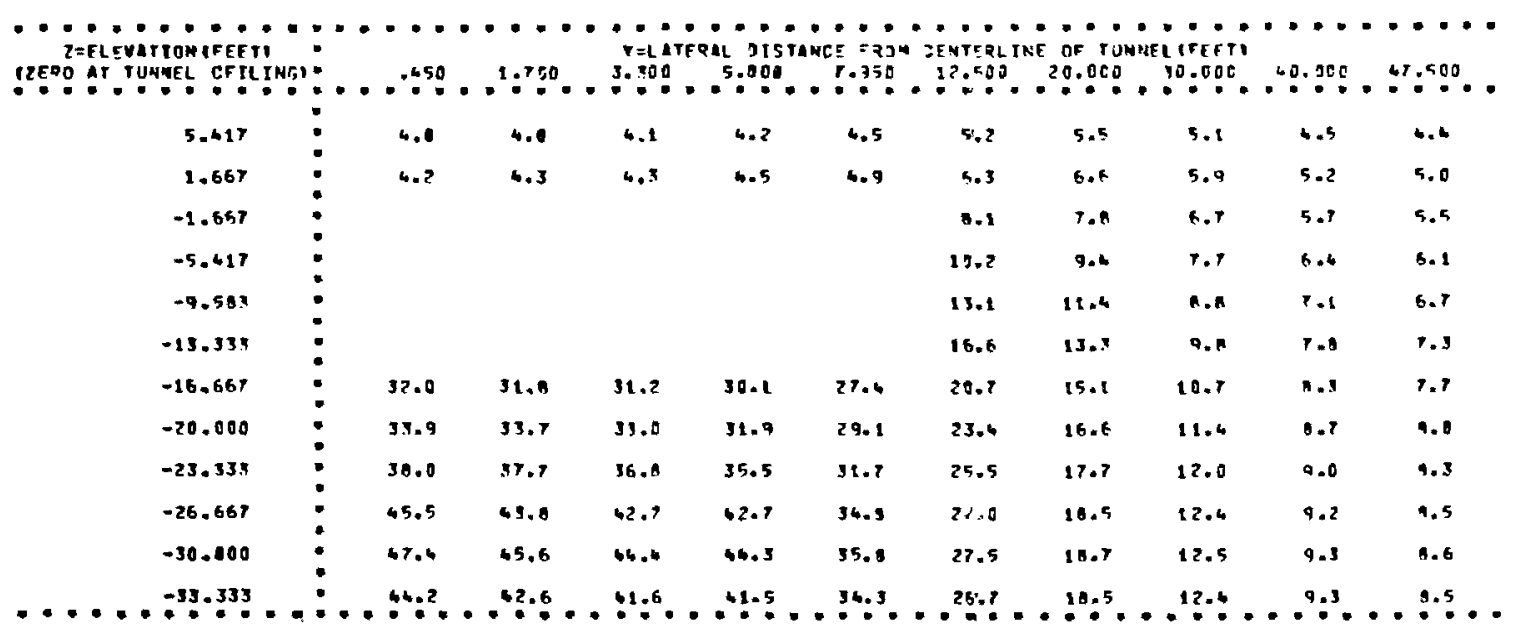


T.MIIE:B-S

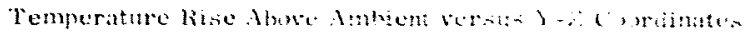

Time (rrs) $\geq 0$

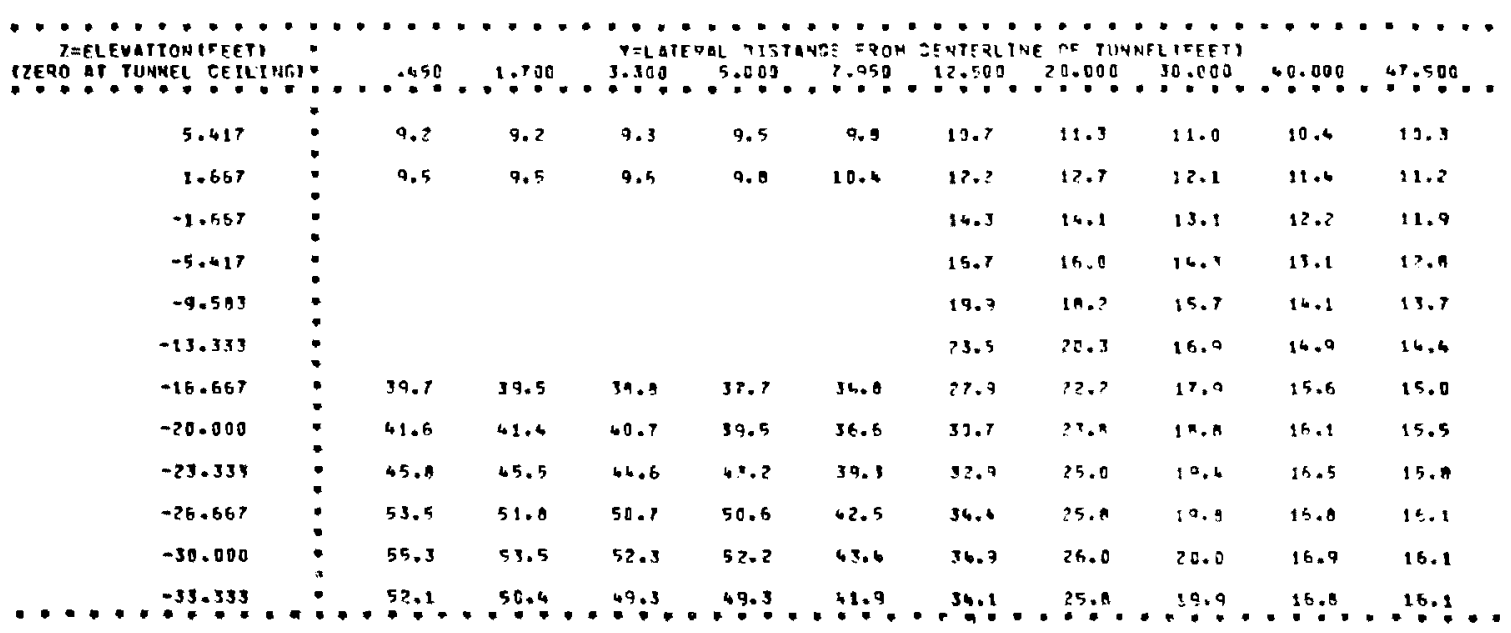




\section{TABLE B--}

Temperature Rise Above Ambient versits $Y-Z$ Coordinates

Time (rrs) $=3.0$

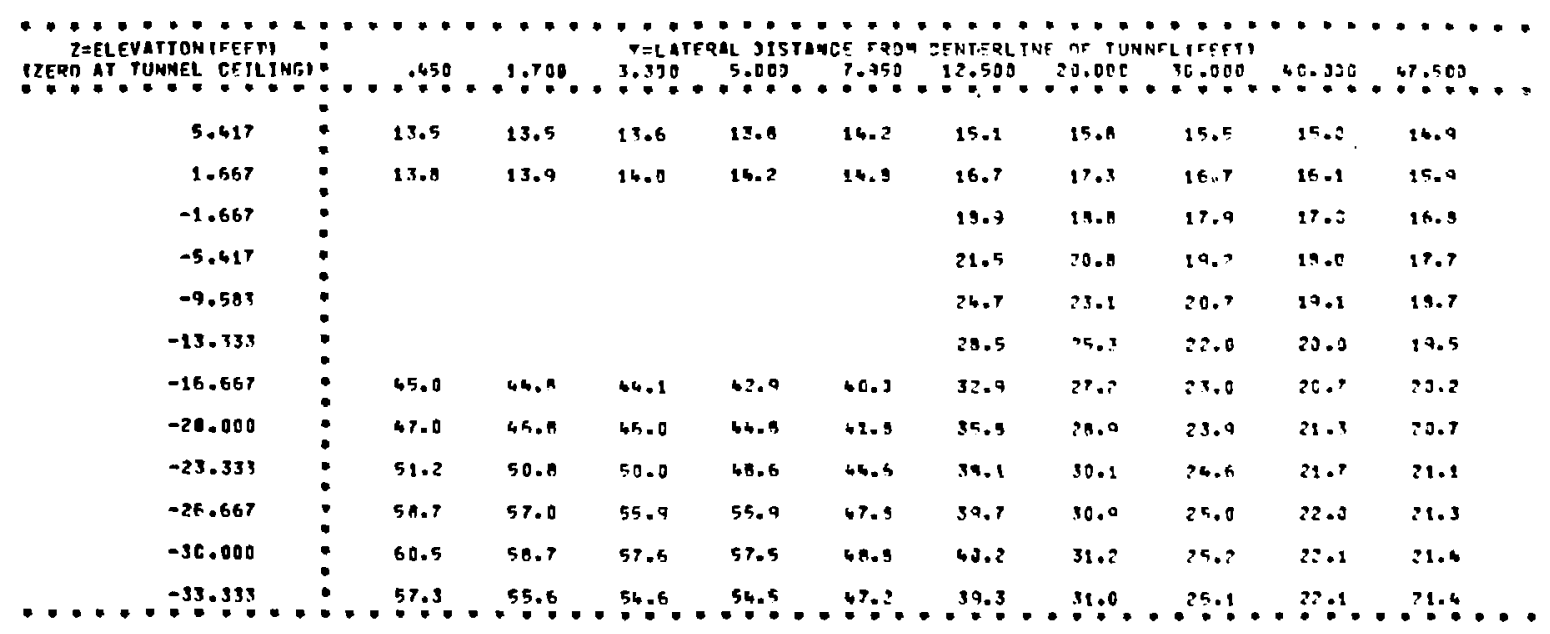


T1 1 1 $1: ?:-5$

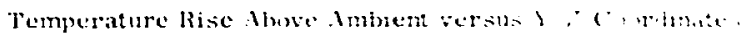

Yime $(y-1) \div 0$

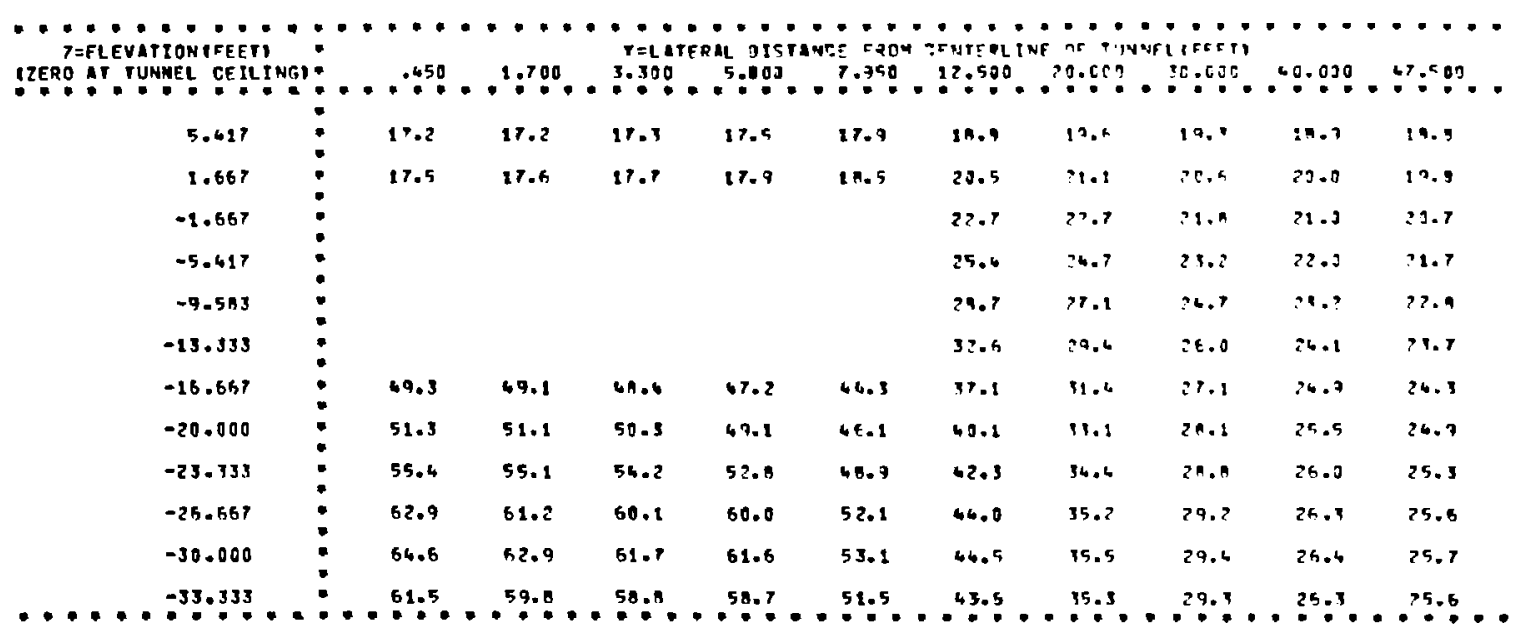


TAI3LE: $13-6$

Temperature Rise dbove Ambient tersis $\mathrm{r}-\mathrm{l}$ Courdinatesi

Time (yrs) $=5.0$

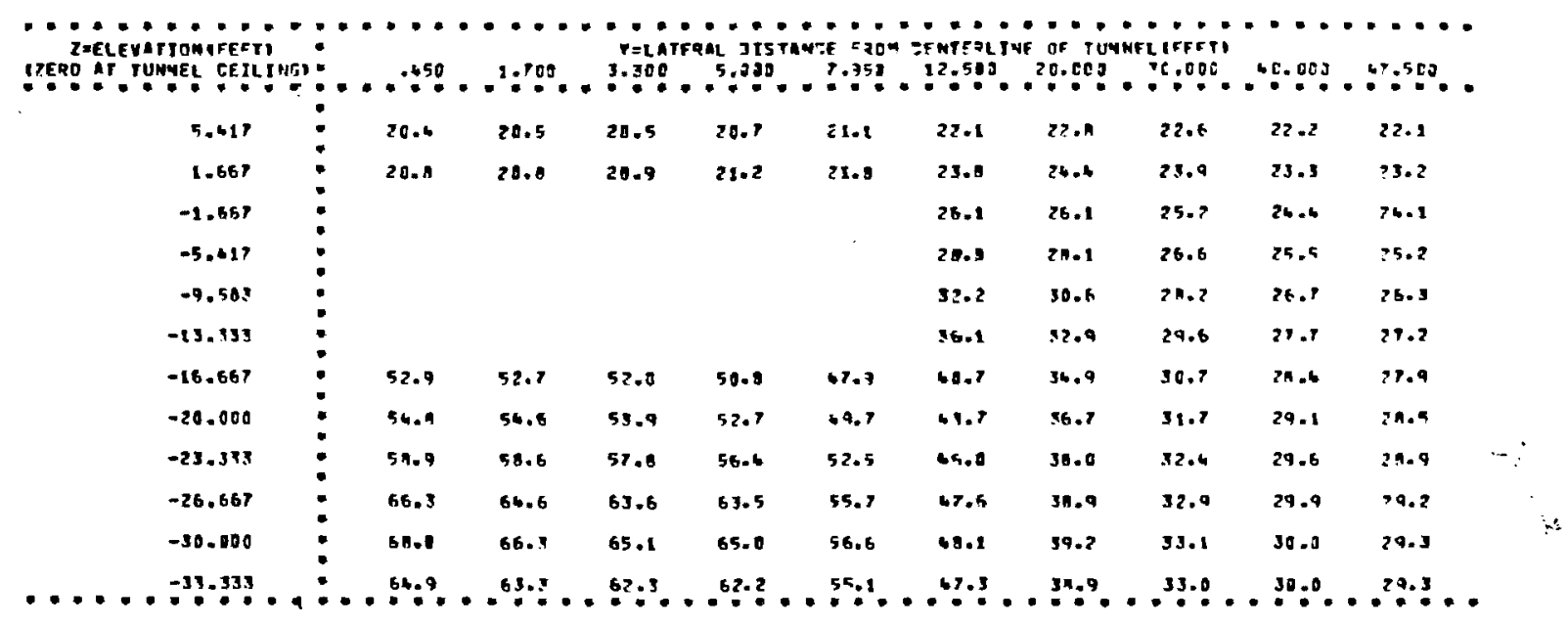




\section{T.|1\}!.: $1 ;-7$}

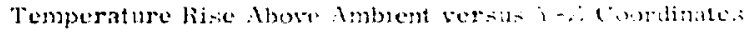

$$
\text { lime (x:s) } 10.0
$$

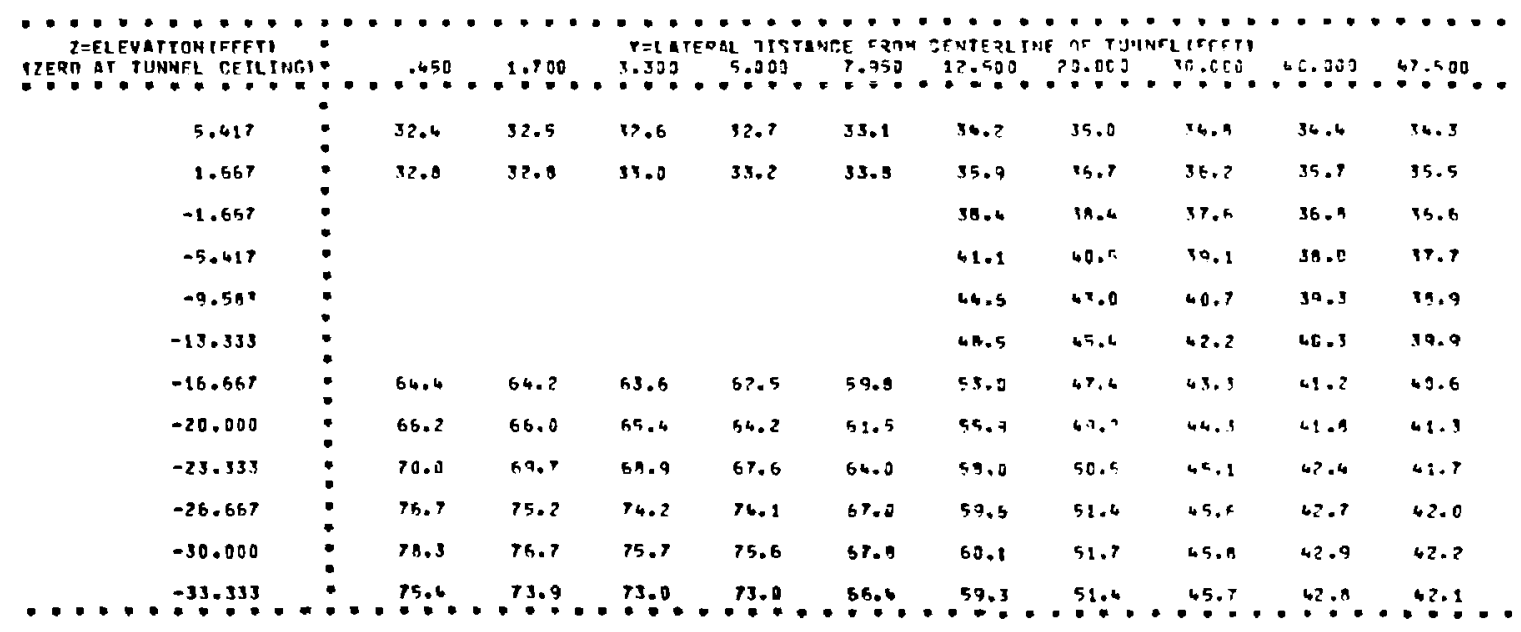


TABIA:B-S

Temperature hist: lbove Ambient versids $3-7$, Coordinates

Time (urs) $\geq 0.0$

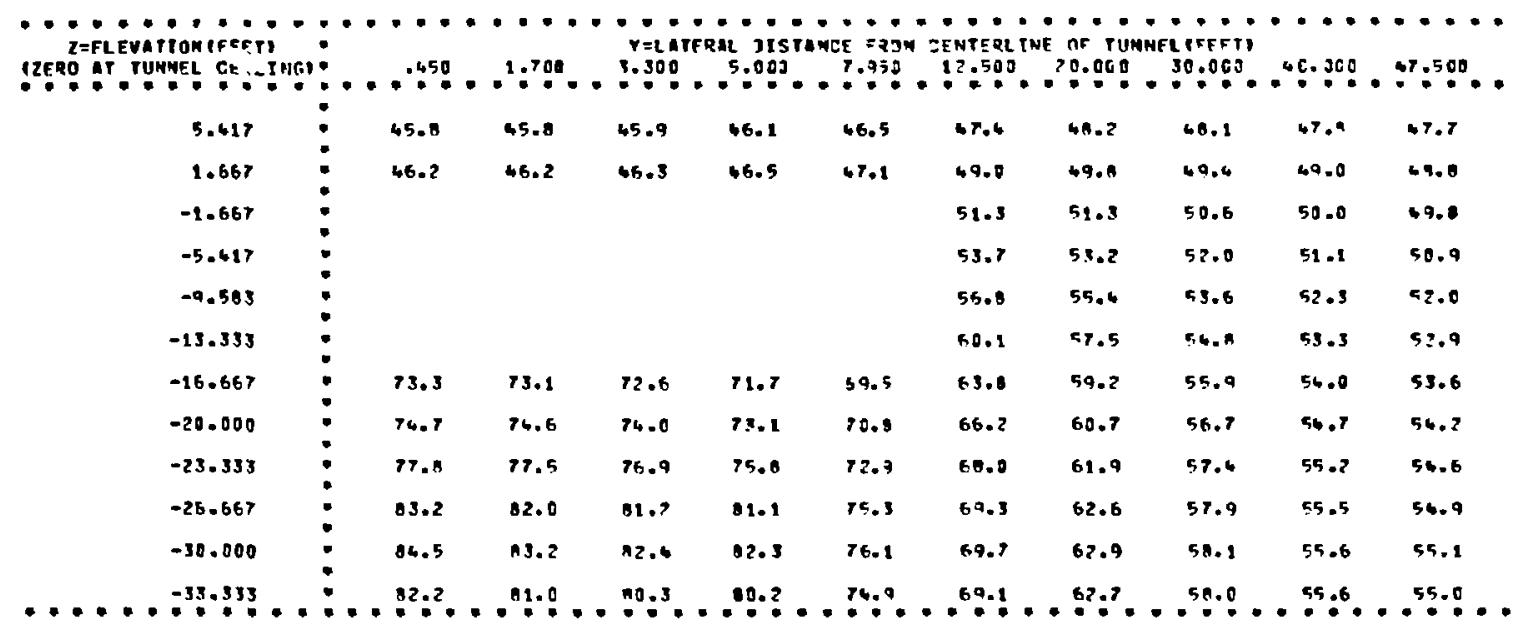


1:1131::13-:3

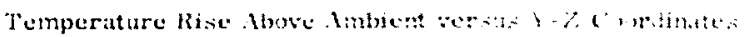

Wi:ne (y.: $: \$ 0.0$

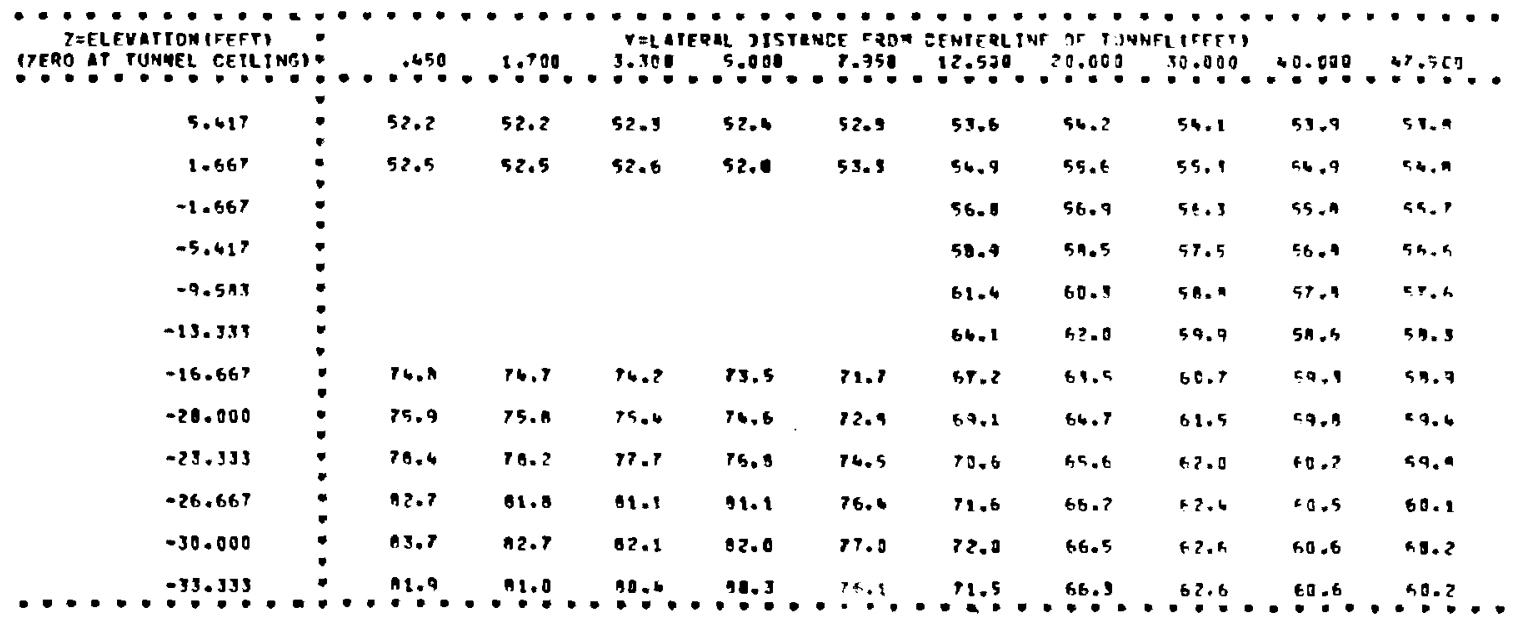


T:MBLE: 1:-10

Temperature Rise Nbove Ambient versis y $-j$ ( onortinates

Time $(y r s): 40.0$

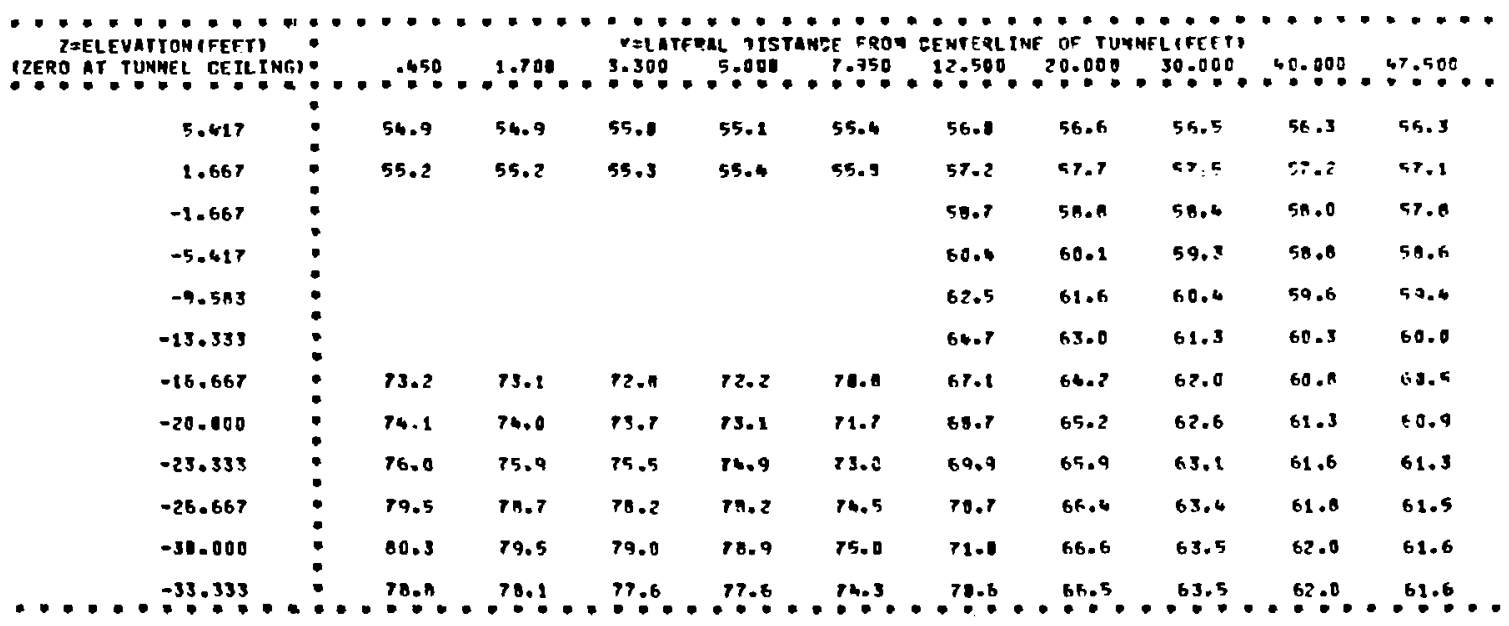




\section{T:131.1: $13-11$}

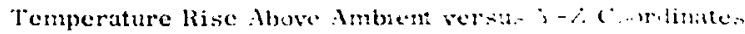

Time $(y \mathrm{rs}) \quad 50.0$

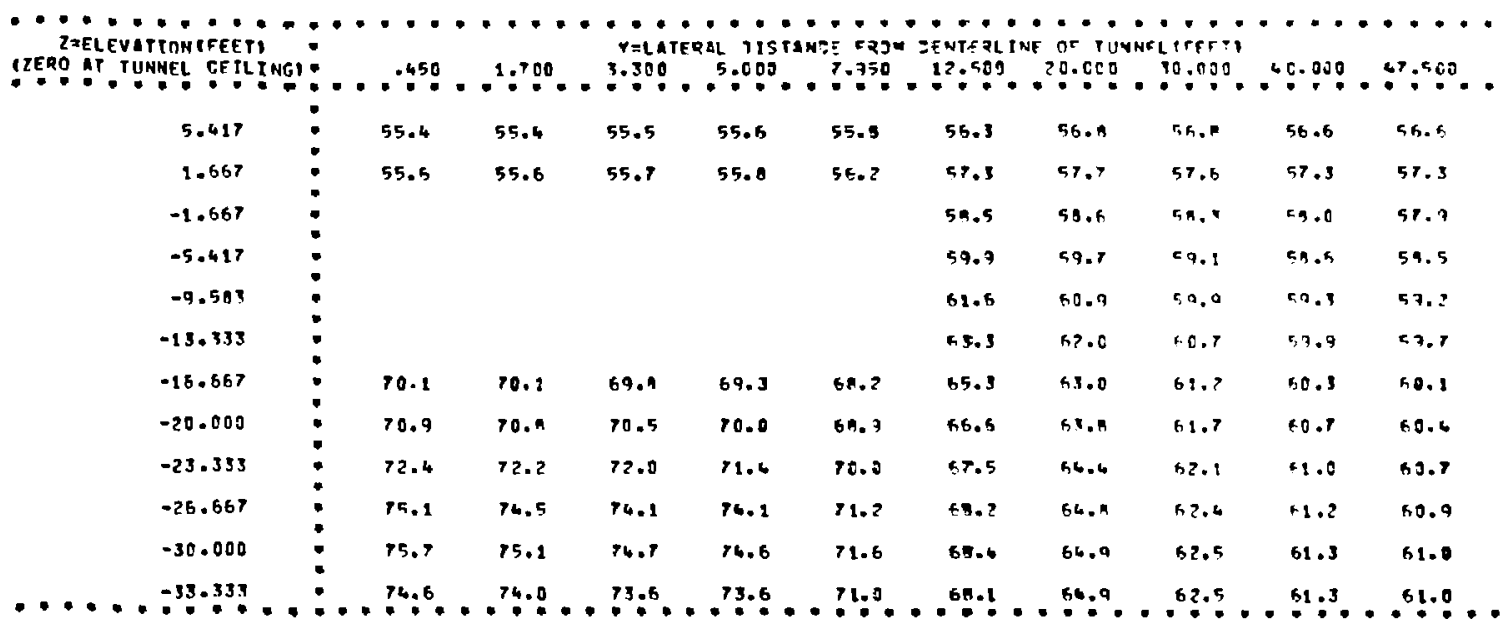




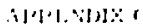

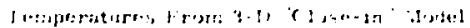

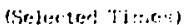

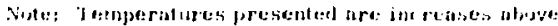
imblent (sere Troxt disc usisiant). 
Correspondence Between Actual and helative Xode Numbers 3-D Close-in Thermal Model

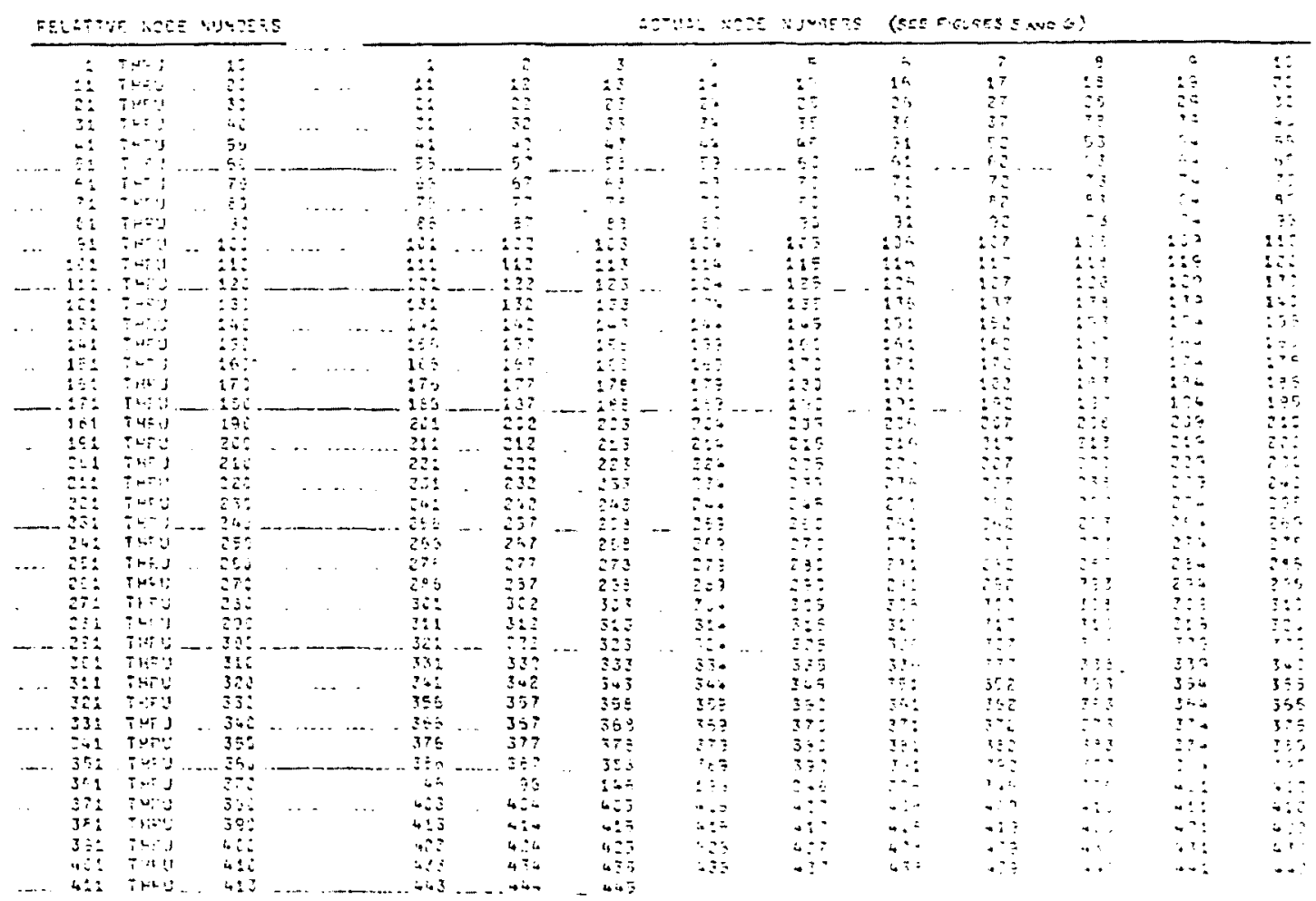


3-D Close-in Thermal Model hesults isr Jul: Waste

prLative none nuvates

\begin{tabular}{|c|c|c|}
\hline I 4 E: & & \\
\hline !' & 35 & $\begin{array}{l}33 \\
45\end{array}$ \\
\hline 41 & ס & \\
\hline $\begin{array}{l}51 \\
01\end{array}$ & $\begin{array}{l}\text { I? } \\
\text { T? }\end{array}$ & $\begin{array}{r}30 \\
160\end{array}$ \\
\hline 26: & ח & $: 23$ \\
\hline & ? 0 & \\
\hline $1 \div:$ & ;? & $: 5,9$ \\
\hline 15! & דו & 10 \\
\hline 131 & In & \\
\hline$? 21$ & In & 22 \\
\hline $2 ? 1$ & 17 & $2 \div 3$ \\
\hline $24:$ & r? & $2 \leqslant 3$ \\
\hline $34 !$ & is & 7 \\
\hline $\begin{array}{l}4 \\
3 \div 1\end{array}$ & 17 & $? 2$ \\
\hline $7 ? 1$ & חז & 36 \\
\hline 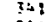 & ז' & 26 \\
\hline $3+1$ & I? & \\
\hline $70 ?$ & I? & 631 \\
\hline & & \\
\hline
\end{tabular}

\begin{tabular}{|c|c|c|c|c|}
\hline $\begin{array}{l}0.0 \\
0.0 \\
0.0 \\
0.0 \\
0.0 \\
0.0 \\
0.3 \\
2.0 \\
0.0 \\
0.0 \\
0.0 \\
0.0 \\
0.0 \\
0.0 \\
0.0 \\
0.0 \\
0.0 \\
2.0 \\
0.0 \\
0.3 \\
0.0\end{array}$ & $\begin{array}{l}3.0 \\
9.0 \\
0.0 \\
0.0 \\
0.0 \\
0.2 \\
0.0 \\
0.0 \\
0.0 \\
0.0 \\
0.0 \\
0.0 \\
0.0 \\
0.0 \\
9.0 \\
0.0 \\
0.0 \\
6.0 \\
1.0 \\
0.0 \\
0.0\end{array}$ & 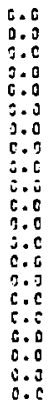 & 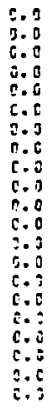 & 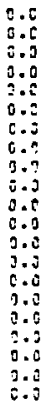 \\
\hline
\end{tabular}

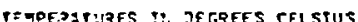

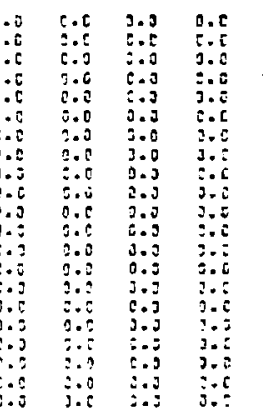

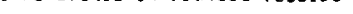

\begin{tabular}{|c|c|c|c|c|c|c|c|c|c|}
\hline 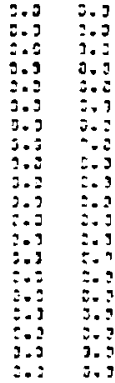 & 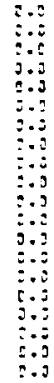 & 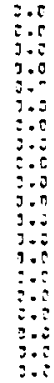 & $\begin{array}{l}0.0 \\
0.0 \\
0.0 \\
0.0 \\
0.0 \\
0.0\end{array}$ & $\begin{array}{l}0.0 \\
0.0 \\
0.0 \\
0.0 \\
0.0 \\
0.0 \\
0.0 \\
0.0 \\
0.0 \\
0.0 \\
0.0 \\
0.0 \\
0.0 \\
0.0 \\
0.0 \\
0.0 \\
0.0 \\
0.0 \\
0.0 \\
0.0\end{array}$ & 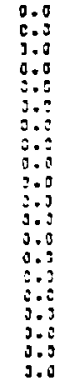 & $\begin{array}{l}0.0 \\
0.3 \\
0.3 \\
0.3 \\
0.2 \\
3.3 \\
2.3\end{array}$ & 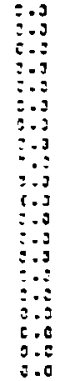 & 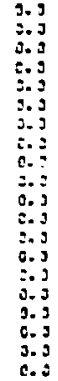 & 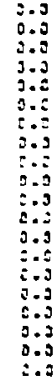 \\
\hline
\end{tabular}

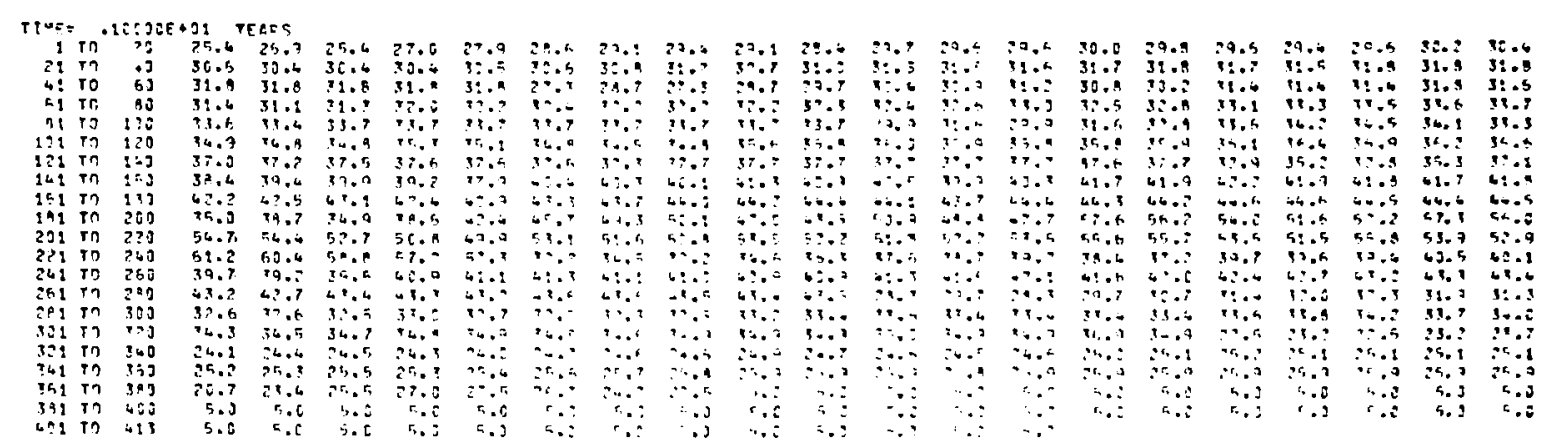




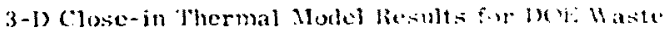

PELA:TVE NITF MaUTED

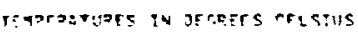

\begin{tabular}{|c|c|c|c|c|c|c|c|c|c|c|c|c|c|c|c|c|c|c|c|c|c|c|}
\hline te & ז & 20 & $3: r$ & $34 \ldots$ & 2.7 & 4.4 & 5.6 & 1,1 & 15,7 & 37,3 & 5.1 & 35,3 & & 17.7 & $37=6$ & 37,4 & 17.4 & 17. & 75.7 & $1 . .2$ & 37,4 & 39.0 \\
\hline 21 & $\begin{array}{l}13 \\
15\end{array}$ & 60 & $\begin{array}{l}x=2 \\
39.5\end{array}$ & 0.9 & $\begin{array}{l}\text { id. } \\
7.5\end{array}$ & $\begin{array}{l}0.3 \\
7.5\end{array}$ & 9.1 & $\begin{array}{l}39.2 \\
36.7\end{array}$ & $\begin{array}{l}34.5 \\
: 4.2\end{array}$ & $\because 697$ & $\begin{array}{l}4.2 \\
4.2\end{array}$ & 37, & $\begin{array}{ll}39.7 \\
58.0\end{array}$ & 10.5 & $\because 2: 3$ & $\because 0$ & 8.5 & : & $\begin{array}{l}39.2 \\
17.2\end{array}$ & & $\begin{array}{l}37,6 \\
39,2\end{array}$ & $\begin{array}{r}32.5 \\
\hdashline-3\end{array}$ \\
\hline al & T? & 00 & 39.2 & 14.7 & 29.9 & 90.7 & 39.3 & 48.2 & 39.7 & 97.3 & 30.6 & 30.3 & $\therefore$. & $6=x$ & $\therefore .7$ & 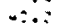 & $4: 5$ & 650 & $4: 2$ & $\because \because$ & $4: .3$ & $\begin{array}{l}7.7 \\
4 ? .9\end{array}$ \\
\hline $9:$ & is & $\because \geq 0$ & $4 ! 3$ & $4 \div .1$ & $\therefore \cdot 4$ & $6: 6$ & 41.4 & $\because$ & $4: 4$ & $4: \div$ & & $\therefore \therefore$ & & $3: 1$ & & $\rightarrow 1$ & 45.3 & $\therefore$ & $4: 0^{\circ}$ & $\because:$ & $6: 5:$ & $\because 9$ \\
\hline 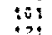 & $T^{n}$ & 123 & $4 ? .5$ & 42.5 & $6 ?, 4$ & & 478 & & $\because \div !$ & B. & & $\therefore$ & -5.5 & 69.5 & $=1.4$ & -5.5 & $-5,5$ & 4.7 & 46.? & +6 & 61.7 & $\therefore \ldots$ \\
\hline 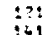 & in & $\because 2$ & $+\cdots 6$ & 64.9 & 65.2 & $\div 5.3$ & 45.7 & $(x,+?$ & 45.2 & 15.4 & 4.4 & -3.3 & 65 & $6 \div-6$ & -4 & $4 . x$ & $6 \div .6$ & 4.). & $4 \cdot$. & +6.9 & 4i." & $6 \ldots 5$ \\
\hline$\vdots \vdots$ & TM & $\vdots<9$ & 45.9 & 47.8 & 47.5 & $-k .0$ & 65.5 & $6=$. & 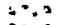 & 3.7 & $=9.9$ & .9 & $69=2$ & 47. & 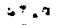 & .0 .8 & 63.5 & 63.9 & 6.5 & $b$. & 63,1 & $\cdots$ \\
\hline :5: & T? & $: 30$ & 40.8 & $\because 0.1$ & $52, t$ & $=0.3$ & 53.5 & 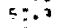 & $5: . ?$ & $z: .5$ & 51.7 & $5: .7$ & $=$ & $5:-1$ & $=\because .2$ & $5: 00$ & 41,1 & $\because \because$ & $5 \%$ & S., : & $5 \div 2$ & $\because \therefore$ \\
\hline $10 i$ & חז & 230 & $4: .4$ & $4 k .2$ & 62.3 & 44.0 & 42,9 & $5 \% .3$ & $x, 5.2$ & 57.6 & $5+.6$ & 33.3 & 1.2 & ar.. & $=\pi$ & $16, A$ & \&. 1,4 & $11 \ldots$ & 59.7 & 53.8 & 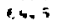 & A. \\
\hline $2: 1$ & $\mathrm{r}$ & נינ & 62.1 & $E: \ldots$ & $\epsilon \therefore 1$ & $\because 2$ & $\because+$ & $\therefore=5$ & 57.3 & $=9.2$ & & $=3.5$ & $=7,2$ & 53,5 & $6: 97$ & :.: & 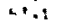 & & $\cos .9$ & 4.1 & $\therefore:-$ & $\because \ldots$ \\
\hline$\because 1$ & in & $8 \div 5$ & 39.7 & $\Rightarrow 9$ & an.: & 64.7 & $5 . .7$ & 30.4 & $6 \because 9$ & $3+5$ & $\rightarrow$. & 69.9 & -5.5 & $\therefore-:$ : & 65.4 & $60^{\circ}$ & 46.5 & $67:$ & 4.9 & 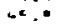 & 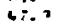 & $\therefore$. \\
\hline $24:$ & $\because$ & $i=5$ & 47.1 & $4 x=5$ & LF: & $B x_{2}, y$ & 67.5 & $\therefore 0^{-2}$ & $6 \times .5$ & 47.6 & 8 & $\therefore 1$ & & 42.0 & $\rightarrow 3 .=$ & 42. & $\therefore 4$ & & 5,1 & & & $4 \therefore=n$ \\
\hline$\therefore \approx 1$ & $\therefore$ & {$[93$} & $7: .5$ & 7.0 .1 & $5: .4$ & $5,5.7$ & 53.4 & $=: .2$ & $5: .0$ & $\therefore .7$ & $\therefore 9$ & $=\ldots$ & & $+\ldots$ & $\because \because$ & 7. & 31.6 & $\cdots, 7$ & 33.7 & 3.4 & $\because$ & $3 \%$ \\
\hline$P: 1$ & Tn & $x$ & 19.7 & $+9, k$ & 37,1 & 60,7 & $\therefore 9,7$ & 3.9 & 77.5 & 57.9 & 62.5 & $\therefore: .6$ & $\because \therefore 2$ & $L=;$ & $\therefore .+$ & ni.; & -5.7 & 63,1 & $6 ! .1$ & $\therefore \ldots$ & $6: 9$ & -1.7 \\
\hline 3) & 17 & 920 & 61.5 & 61.9 & $4 i, i$ & $6 ?+1$ & 69,1 & $6 ? .1$ & 41.9 & $\because .2$ & & & & 67.2 & & 6.1 & $\therefore \therefore$ & & & & & \\
\hline $3 ? 1$ & 10 & 549 & 11.2 & 1.5 & 19,5 & 19,6 & $7 ! .1$ & $5: 99$ & $3: .9$ & י, & .5 & $1: \ldots$ & $1: 9$ & צ'. . & & $\because .7$ & $1 ., 3$ & $\because \cdots$ & .5 & & $\therefore 3$ & 9.3 \\
\hline $34:$ & $?$ & 360 & 72.4 & $\because \div 5$ & $12+7$ & $7 * 5$ & $\because, 6$ & $5 ?$ & 7.2 & II. J & . : & : : : & 11.: & 7.9 & $1 \geqslant$ & 93.1 & 11.1 & 17.8 & N:I & ?. & $\because,:$ & $9:$ \\
\hline & 13 & inj & $\therefore 9$ & $\because .7$ & $\therefore .9$ & 34.4 & 76.9 & 74.1 & $\therefore .7$ & $\because, 5$ & 3.7 & $1: .7$ & 120 & ; & 17. & $1: .7$ & $: 0.7$ & & 2. & $\therefore 7$ & : 5, & . \\
\hline 74: & $\because 1$ & $\therefore 50$ & $1 \% .7$ & $: 0.7$ & $\therefore 7$ & 13.7 & $: 9.7$ & $1: .7$ & $: 2.7$ & $::$, & .7 & & $\because:$ & $\because$ & & $\because \therefore$ & $\because \therefore 7$ & $\therefore A$ & $1 \therefore .7$ & $\therefore \therefore$ & IC., & $: 2.1$ \\
\hline $4 \therefore 1$ & in & +11 & 0.7 & 10.7 & $16=:$ & $i 6.7$ & $: 9.7$ & 13.7 & $: 3.7$ & $\therefore .7$ & 3.7 & & & $\div \quad 7$ & & & & & & & & \\
\hline & & & & & & & & & & & & & & & & & & & & & & \\
\hline & T & 2 & 37.7 & 3.7 .6 & 37.0 & 9.6 & 60.0 & 1.3 & $: .9$ & $? ?$ & 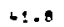 & 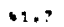 & .5 & $+i .6$ & -2.6 & -2.9 & $\rightarrow \cdot t$ & $a ?, 6$ & $\because 21$ & .6 & 43.2 & +1.2 \\
\hline$\therefore i$ & 17 & 69 & 6.4 & $6 ., 3$ & & $\Rightarrow$ & 7,5 & . & 7.7 & $6 . j$ & 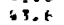 & & & $-6,6$ & 6. & $\ldots$ & 6.7 & & 6.6 & &. .7 &. \\
\hline-1 & in & 62 & $44+9$ & $6 \ldots$ & $\ldots 7$ & 66.7 & $+\ldots .7$ & 37.9 & $4: .6$ & 7.7 & 61.6 & $\because *$ & $\because$ & 47 & $b \div$. & 27.4 & $\because 1 .:$ & & $\ldots$ ? & 64.2 & $-6,7$ & Q e. . \\
\hline$\div:$ & iา & aj & 45,2 & is.? & $\| \bar{c}$ & 64.7 & As, & $4 ? ?$ & 45.4 & 65.3 & $=:$ & $\therefore=$ & & $s=.5$ & $\therefore$ & $6 \pi .4$ & $\therefore 7$ & & LG.: & & 64.4 & 46.4 \\
\hline 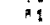 & in & $1: 9$ & $6 \leqslant .5$ & 4.: , & Lh. F & $\therefore 4.5$ & when of & 6r. & $\because \pi, k$ & $b=9$ & 8 & $\therefore h+i$ & & $2 . .2$ & & $\ldots x$ & $\therefore \therefore$ & & 7.2 & & $4 f_{1.2}$ & 4.9 \\
\hline $1: i$ & T9 & $1>0$ & 47.7 & 47.7 & 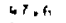 & Ga. 2 & 67.3 & $\therefore 7$ & $\because .1$ & 47.5 & 64. & $4 \circ$ & 169 & $\ldots 7$ & $=1$. & $\angle A$, & $-9, \cdot$ & -4.7 & $\rightarrow$ & $\therefore .7$ & $62 .:$ & $=7.5$ \\
\hline$\because$ & חו & 100 & 49.7 & 50.1 & C., T & $a_{1}, \ldots$ & 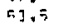 & $\therefore r$ & $5: 3$ & $r_{5}, 3$ & & $\because$ & & $\because ;$ & $=3.6$ & ct.r. & $\therefore \mathrm{H}$ & 4.94 & 49 & & 47.7 & 6.9 \\
\hline 141 & 70 & 150 & 51.1 & $=7.1$ & 9366 & $5: 7$ & 50.4 & $5 \div 1$ & $\because:$ & $=7.3$ & $\because \neq i$ & $\therefore .5$ & $\because$ & $\because$ & $\because$ & 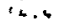 & . & & 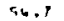 & & 14,4 & a6. 6 \\
\hline in: & כד & 180 & 56.7 & 59.2 & 55.4 & ${ }^{2 r}, 1$ & 59.6 & $5 x .5$ & at., ! & r.h. & i & $\therefore$ & $\therefore$ & . & $\infty$ & 1.2 & 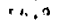 & $5 \cdot, 1$ & $5, .3$ & $\therefore 7$ & $r 7,1$ & $5^{\circ}$. \\
\hline 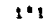 & T? & 3 & 47.4 & $=1.1$ & 47.5 & 51.1 & 5.6 .9 & 5.1 & \pm 1.4 & $\because * 1$ & & $\therefore 2$ & $\because$ & 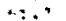 & $4 \therefore$ & 43.4 & $=7$ & & 4.. & & क7. $=$ & 49.1 \\
\hline 201 & T) & $2 ? 0$ & hk. $=3$ & EF., & & $=9.1$ & $<2, ?$ & $5,5,2$ & & $\therefore ?$ & 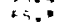 & 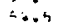 & $\therefore$ & $\because,{ }_{n}$ & & $\because:$ & 1.1 & 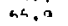 & 5.2 & $\therefore$ & $x x+1$ & 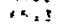 \\
\hline i: & $T$ & $\therefore 40$ & 73.5 & $\because$ A & 71.7 & 60,5 & $5,9,7$ & $+4,9$ & $u$ & $\therefore+.9$ & $\Rightarrow$ & $=9.9$ & $\because \because$ & $=\therefore$ & $\because \because$ & $\because "$ & -7 & $\therefore \therefore$ & $>1$ & 4 & 53.2 & $4 x$ \\
\hline 242 & T? & 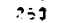 & $\Rightarrow \because 7$ & $5: .7$ & 57 & $=2+4$ & 5 T.4 & $=1.0$ & $5 x, 5$ & $\therefore 1.3$ & $\sum \bar{z}=6$ & $\div \ldots$ & $>=9$ & $\therefore:$ & $\therefore$ & $\therefore \ldots$ & $\ldots$ & & & $=r_{8}, 4$ & G., 9 & as. \\
\hline 25: & T & $\therefore 9$ & 55.6 & 55.2 & 55.0 & cr. a & -5.7 & rna: & sh.: & {$[7.3$} & 55.2 & $\div \quad 3$ & $\therefore \quad \therefore$ & $4 \rightarrow$ & $\therefore=$ & 69 & $\therefore 1.4$ & +1.7 & 64.5 & . . & & $4 .-1$ \\
\hline $2 \cdot 1$ & T? & 139 & 67.0 & 45.0 & $65 . c$ & $15 . \div$ & 45.2 & 4.5 & 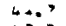 & $\because .7$ & $-t_{1}, t$ & & $\therefore$; & $\therefore$ & $\therefore$ & $=\ldots$ & $\because 2$ & $-4-1$ & $44 . ?$ & & $* * .1$ & 2.6 \\
\hline \pm 2 & I & 1? & $4+.7$ & $4 r_{1} .9$ & 47.1 & 47.2 & 4.7 & 4.7 & 47.7 & $\$ 7.1$ & $\rightarrow x$ & & & & $\because$ & $\because$ & $\because$ & & $\mathrm{F}, \mathrm{s}$ & & 32 & $1 i$ \\
\hline $5 \geq 2$ & TI & $I+i$ & 2E.? & 76.6 & Ia.h & 14.5 & 35.2 & $x, .5$ & 15.4 & 19.7 & $3 \% . z$ & 11,1 & Bn. & $\because \ldots$ & $x$ & $\because, 1$ & $\because .4$ & ". & $x .6$ & & 17.4 & $\because .4$ \\
\hline 141 & ח ז & $k<$ & 37.5 & $\because$ & $\because .7$ & 77.5 & $\because, A$ & 7.9 & $\because \therefore$ & $\cdots .1$ & $\because \ldots$ & $\because *$ & $\because \ldots$ & $* \therefore$ & . & $\because \cdots$ & 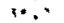 & $\because \ldots ?$ & & & $3 a_{0}:$ & $\because \ldots$ \\
\hline & 19 & ? 9 & 19.3 & & 34. & 37.7 & & 9 & 1 & $3:$ & & $1 \cdots$ & & & & $: x$ & & & & & $1 . .4$ & 10.4 \\
\hline 101 & 17 & $=\geqslant 0$ & 15.6 & !द. & $: 4.6$ & $: 5+5$ & $: 5.5$ & 15.6 & $: 7.5$ & $: 3.5$ & 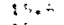 & : & & *. & 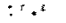 & $:^{\tau}$, & $: \div .5$ & $: 4.2$ & $: c .6$ & $\because t$ & $\neq=5$ & $: \therefore-5$ \\
\hline & TO & 613 & 25.5 & 15.5 & 15.4 & 15.5 & 25,6 & $: 5.5$ & 5.13 & $!^{r}, x$ & 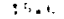 & : & $: \ldots$ & $: \because$ & $:^{\mathrm{t}} \cdot$ & & & & & & & \\
\hline
\end{tabular}


3-J) Close-in Thermal Model hesult if for [X1: Histe

FELATIVF NODE NUMBFES

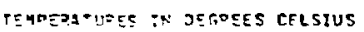

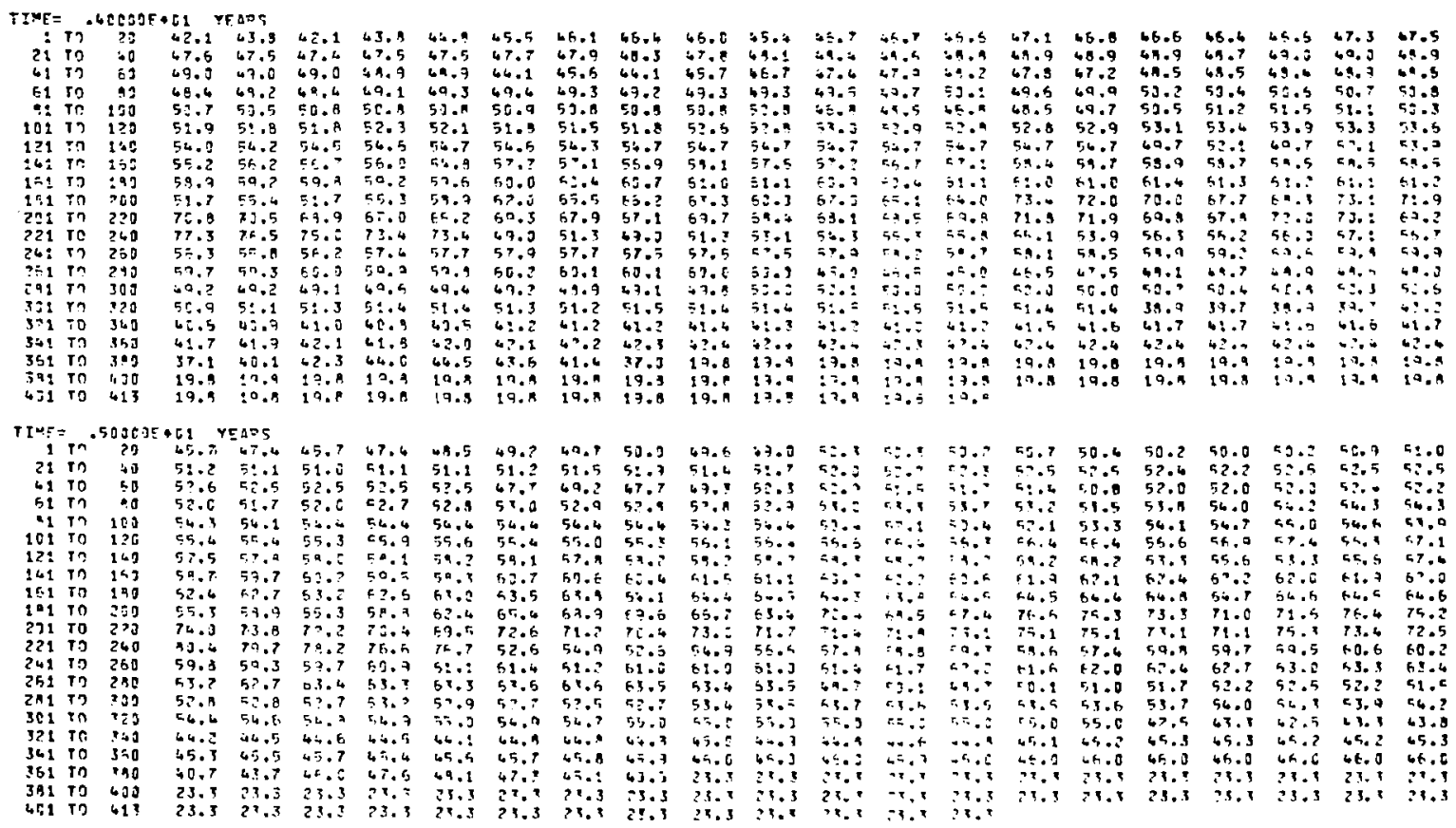




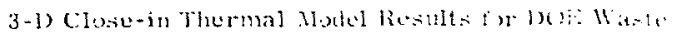

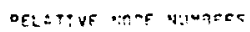

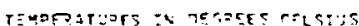

$\operatorname{TACL}=.75 \cos E+01$ Yes?

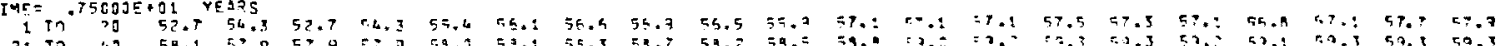

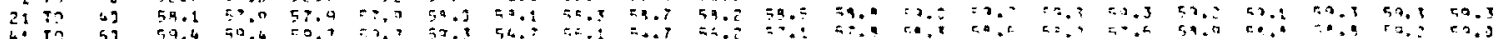

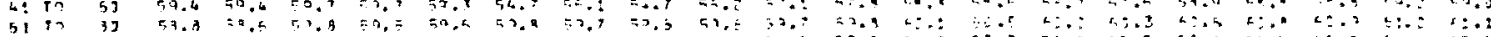

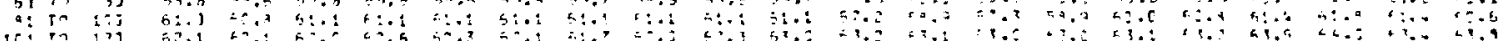

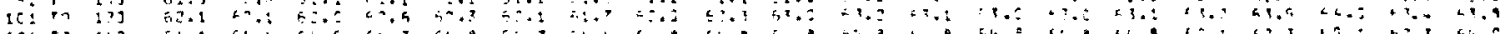

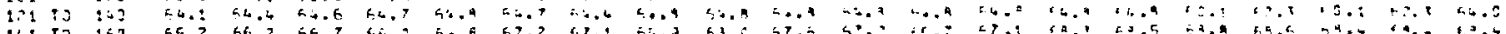

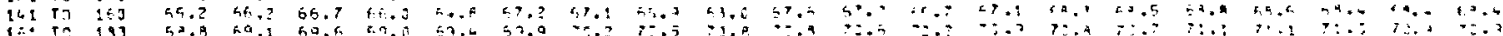

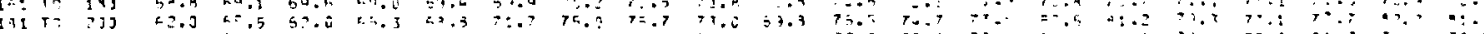

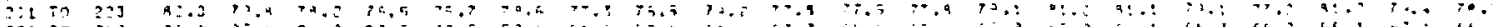

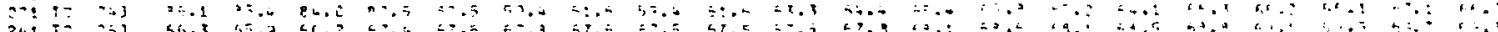

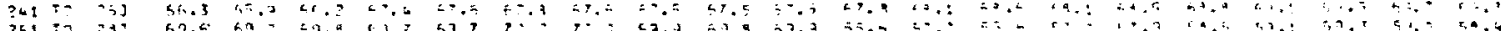

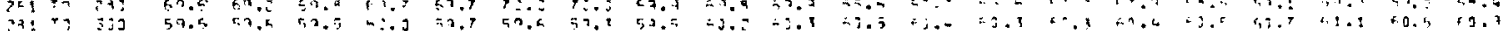

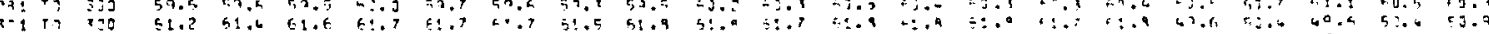

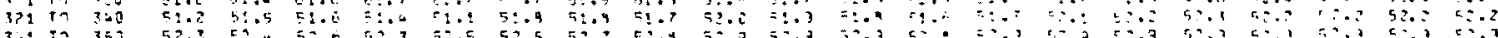

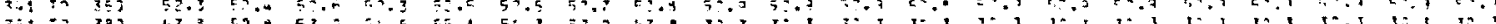

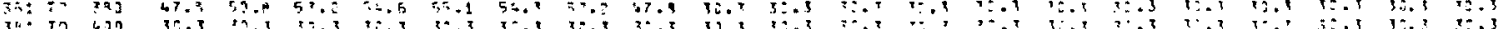

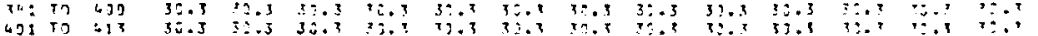

\begin{tabular}{|c|c|c|c|c|c|c|c|c|c|c|c|c|c|c|c|c|c|c|c|c|c|c|}
\hline 1 & T & 230 & +67.7 & की. 3 & 57.7 & 59.3 & $63 . ?$ & $\in[, T$ & 51.5 & $F:$. & 91.4 & $s: 0$ & 53.3 & $=0$ & $\because:-0$ & $\because 3$ & 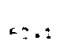 & $n: .7$ & $6: \ldots$ & $5: \ldots 7$ & 67,5 & $\Leftrightarrow: 7$ \\
\hline 21 & 17 & 4] & 62.7 & $5: 2$ & $6 ? .7$ & & 62.9 & & $5 \times 1$ & E. . & $5,5.3$ & & & $A x a$ & 59.7 & & $\therefore$ S : & 4,2 & & $*, 2$. & क.4.: & $E<=1$ \\
\hline 41 & in & 50 & & 66.1 & E & & 64,1 & 50.6 & $a \div, 0$ & $\therefore 0.4$ & & $=2,3$ & $s ?$ & M.: & $=1.4$ & 13.8 & 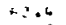 & $\cdots, n$ & 59.4 & 47.5 & 36,3 & 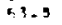 \\
\hline $5 i$ & in & 90 & 53.64 & 59.3 & $? .5$ & 54.2 & 54,4 & 56.5 & 46.6 & 5.5 & $\therefore, 6$ & 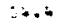 & 545 & $\ldots$ & $=1$. & $14.0^{\circ}$ & . . . .5 & $4=.1$ & $65_{1}, 6$ & 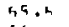 & $=5.7$ & $x=.9$ \\
\hline 9 I & Tn & ננ & 65.7 & 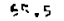 & 55.8 & $55, A$ & $6=.9$ & 55.9 & $\therefore, A$ & 5.1 & 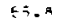 & 57.1 & $\because 2$ & 23.7 & $\therefore:$ & * : & $\ldots$ & $1 \therefore$. & ; & 26.4 & af.? & c. . \\
\hline 121 & 17 & 289 & $6 \leq-7$ & SR: & 5.6 & Gi: $=$ & 45.9 & $E, E, 1$ & Enis & $\therefore x=5$ & 57.6 & 57.5 & & ir.a & $\therefore$ & +8 & $\therefore$ & 1.7 & $\therefore i$ & 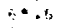 & & $5, \cdots 3$ \\
\hline $1: 1$ & Tา & 100 & 59.7 & $59.1)$ & $89 .:$ & $50, ?$ & 50,7 & 69.2 & 53.0 & 6.7 .3 & 59.9 & $E^{2}, 1$ & $\Leftrightarrow 3.4$ & 0,1 & $\therefore .1$ & $\because, 1$ & 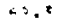 & G. . A & 4. . & sis. & 4.7 & $1 \cdot .6$ \\
\hline 161 & 77 & $1 \in 0$ & 40.5 & 73.9 & & 76.7 & $=7.4$ & & 11,9 & & & & & $\because:$ & $\therefore . t_{5}$ & $\because y$ & $\because 2.7$ & & $\because 4$ & $\because$ & $\therefore 9$ & $72 . \mathrm{A}$ \\
\hline 151 & 17 & 190 & 73.1 & $1 * 0$ & 73.9 & 77.4 & $7 \pi, 9$ & 74. & 96,6 & $7 \div$. & 73.8 & 75 & 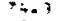 & 76.5 & $83 .:$ & ir.: & 15.0 & 5.3 & $7 r .$. & $\because<.2$ & ?5.: & $7 \times .7$ \\
\hline 191 & 10 & 200 & SE.S & 69.7 & 55.5 & F.१. & $?+1$ & $\because 5.7$ & 77.1 & $=3$. & $7 . i$ & $7=1$ & $8: .5$ & 7.7 & 77.7 & $\because 2$ & $a \div .0$ & म 7.: & $4 ! . ?$ & 91.5 & 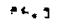 & .6 .7 \\
\hline 721 & 13 & $22 \mathrm{~J}$ & 13.0 & $\because 5$ & 1 & $a c=5$ & 70.7 & 32.5 & 91.7 & $\therefore \div$ & $\because 3$ & 91.7 & & 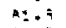 & -3.3 & a & 94.9 & 43,3 & & & & 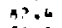 \\
\hline $22:$ & IT & 240 & 99.7 & $70 . i$ & 8. 6 & A5. Z & 35,3 & & $4 \cdot x$ & $\Leftrightarrow$ & 3 & $E$ & & 10.2 & 73.3 & 9,9 & $\therefore 4.5$ & $7 \because 9$ & $\cdots .7$ & 76.5 & 71.5 & $7: .1$ \\
\hline $2+1$ & T & 265 & $7 \hat{n}$. & $72 .$. & 79.7 & 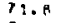 & 22.6 & & & & & & & $7 \because 4$ & -3.2 & 79,5 & $: 2.8$ & & 83.5 & 78.5 & 74.3 & R..I \\
\hline ?an 1 & חז & נד? & $7 \times .7$ & 73.5 & 76.1 & $7 L, 3$ & 74,6 & 14.3 & 20.8 & $r$ & $r+1$ & ib. & & $\therefore: \therefore$ & $\Rightarrow 3.5$ & $5: \ldots$ & $\therefore 7$ & .1 .1 & $=1$. & $5=1$ & $+\pi$ & $t ? . ?$ \\
\hline $2 \times 1$ & T? & 303 & 64.7 & 46,5 & $5 \%$ & 5.7 & 8.4 .5 & 56 & $5,4.1$ & $c:$ & 56.9 & {$[3.3$} & & 5.2 & $6 \div .2$ & $E r, 1$ & 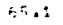 & $\leqslant:$ & 5.5 & $6.5,9$ & $55 \ldots$ & +5.6 \\
\hline $3 \mathrm{Cl}$ & 17 & $\geq>0$ & 55.0 & 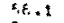 & $a=5,3$ & $F F=4$ & $r_{3} c_{6}, \ldots$ & $5 E .3$ & E, S. 2 & se. & $3^{2} \cdot 6$ & 6. & & Sh. 5 & c... & $6 .$. & & 54,9 & Fr. & $\because \therefore . .3$ & .5 & 5, ᄂ. . ? \\
\hline 321 & סז & 543 & $5 \% .3$ & $5 \in .5$ & $5 \pi .6$ & 56.5 & $56 . ?$ & 55.9 & 54. & $ৎ ; .7$ & $=7$. & 55,1 & & $\therefore<$ & $=-7$ & $a_{7}:$ & $5 \% .2$ & $\because{ }^{4}$ & 57.7 & 57.1 & 57,? & 57.2 \\
\hline 341 & TI & 35: & 97.2 & & 7.5 & 51.1 & 57.5 & & & 5.8 & & $; 7$ & & $=7.7$ & $\therefore 7$. & $=-4$ & $\therefore A$ & $=9.7$ & $\therefore \rightarrow$ & & $=7.3$ & 57.1 \\
\hline $35:$ & I? & 제구 & 59.8 & $\because 0$ & $5 \% .0$ & e & $5 \% 1$ & & 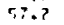 & $=2.1$ & & 53 & & 5,7 & 15.7 & $3 \%, 7$ & $7^{x}, 7$ & $\therefore$ & $y$ & $p r .7$ & & 35.7 \\
\hline 3BI & Tכ & $4[3$ & 35.7 & 35.7 & 35.7 & $\pi .7$ & 75,7 & $=7$ & 35 & 15.7 & 35.7 & 35 & & $r=.7$ & $\because \div .7$ & $\because=, 7$ & 75.7 & $\because$, & 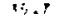 & 35.7 & sद, & p. 7 \\
\hline & in & 412 & .15 .7 & 35.7 & 35.7 & $x x_{*}, 7$ & $8 r_{3}, 7$ & 35.7 & 5.7 & $\{\%, T$ & $x=7$ & $3=.7$ & $x c_{1}, i$ & $5, ?$ & $\because$ : & & & & & & & \\
\hline
\end{tabular}


3-1) Close-in Thermal Model Results for DOE Waste

FELATIVE NOTE NUMAETS

redPERATUES IN DEgOEES CELSIUS

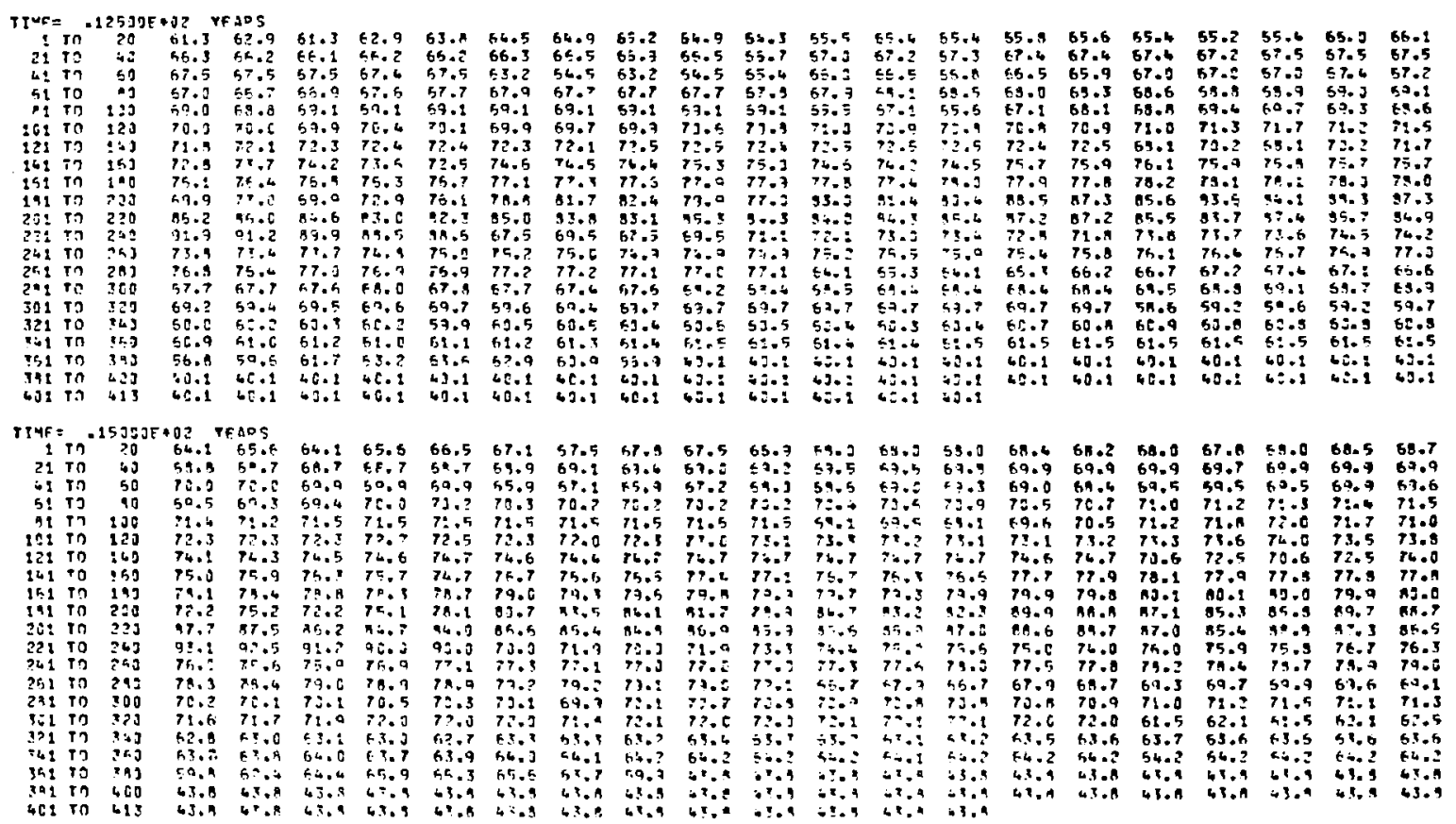


3-1) (lose-in Thermal Model Result s fin 13)i: Wiste

Delative hate numitos

T=7DERATUOES TV JETCETS CFLSTIV

\begin{tabular}{|c|c|c|c|c|c|c|c|c|c|c|c|c|c|c|c|c|c|c|c|c|c|c|}
\hline $47=$ & & $\begin{array}{l}167 \\
99\end{array}$ & & & & & & & & & & & & & & & & & & & & \\
\hline $2:$ & $\begin{array}{l}\text { ?? } \\
\text { to }\end{array}$ & $\begin{array}{l}\because 9 \\
\therefore 0\end{array}$ & $\begin{array}{l}<5,1 \\
69, n\end{array}$ & $\begin{array}{l}59.5 \\
57.7\end{array}$ & $\begin{array}{l}5.5 .2 \\
59.5\end{array}$ & $\begin{array}{l}65,6 \\
67,6\end{array}$ & $\begin{array}{l}5.5 \\
67.5\end{array}$ & $\begin{array}{l}59 .: \\
59 .\end{array}$ & $\begin{array}{l}\because 95 \\
\because 9.0\end{array}$ & $\begin{array}{l}54.7 \\
79.3\end{array}$ & $\begin{array}{l}53.5 \\
37.9\end{array}$ & $\begin{array}{l}57.2 \\
73.2\end{array}$ & $\begin{array}{l}57.3 \\
73.5\end{array}$ & $\begin{array}{l}57.5 \\
75.5\end{array}$ & $\begin{array}{l}59.9 \\
73.7\end{array}$ & $\begin{array}{l}50.5 \\
75.9\end{array}$ & $\begin{array}{l}57.1 \\
30.9\end{array}$ & $\begin{array}{l}59.5 \\
72.9\end{array}$ & $\begin{array}{l}34.0 \\
7: 05\end{array}$ & $\begin{array}{l}59.9 \\
79.0\end{array}$ & $\begin{array}{l}60.5 \\
72.0\end{array}$ & $\begin{array}{l}67.5 \\
79.7\end{array}$ \\
\hline $4 !$ & T? & 50 & 76.9 & 76.9 & 73.0 & 70.0 & $7 \%$ & 56.7 & & 55,7 & $5 \div:$ & 53.3 & 63.5 & 72.2 & 75.2 & $(9.7$ & & $72 . .4$ & 73.6 & $\because=6$ & PE. & $7: .5$ \\
\hline in & :7 & 40 & 32.4 & $\because c .2$ & 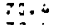 & $7: 0$ & $7: 1$ & $71 . ?$ & $7:-:$ & $7 !: 2$ & & 71.2 & 72.3 & 湜 & 1.9 & $7: .2$ & 15 & $7 ! \cdot ?$ & $\because-1$ & $77-2$ & $\because=3$ & ?. \\
\hline-1 & I & 130 & 72.5 & $7 * 1$ & 72.4 & 72.4 & $7=.4$ & 72.6 & $73 \cdot 6$ & $? ?$ & & 12.7 & 57.1 & 73 & 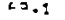 & 7 7. 5 & 71.4 & $\because \cdot$ & 72.5 & $r: 3$ & $7: 5$ & $7:, 3$ \\
\hline $13:$ & Tn & 123 & 7.2 & 7. ? & $73 .:$ & 72.5 & $7 \pi, !$ & $7 \top .2$ & 79.9 & $7 \div:$ & $7 \pi=$ & 7.3 & 76.2 & & -3.2 & $76=$ & 74.9 & $14 . ?$ & 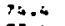 & $7=\ldots$ & $7 \ldots 1$ & 76.5 \\
\hline & In & $1 \div$ & $7: 6$ & $7 \div-4$ & 75.3 & 70.6 & 73.5 & $7=2$ & $7=9$ & & & 8.5 & & & $\therefore \therefore$ & 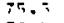 & $\therefore$ & $7: .5$ & 79 & $\because: 5$ & 3.5 & $7 . .9$ \\
\hline $16:$ & Tา & $: \div$ & $7=.3$ & 78.7 & $7: 1$ & 75.5 & $\because 5$ & 70.5 & $7 \cdot$ & 77.5 & 73.2 & 77.3 & 77.5 & 77.9 & 77.4 & ; & 79.5 & 79.9 & 34.0 & $\because .5$ & 10.5 & $7 \because .5$ \\
\hline $25:$ & In & $: 93$ & 73.9 & $=0.1$ & $73 . \pi$ & $i 0,1$ & 73.4 & 1.3 & & $\because 3$ & & $9: 3$ & & & $\because \therefore<$ & $\because, c$ & & 9.9 & .7 & & $\because .4$ & 3.5 \\
\hline$:^{n} !$ & T2 & $?$ & $\because 1 .:$ & $7 c$. & 73.1 & is.? & $\because a$ & $\because: 8$ & 96.5 & $\because .5$ & $\because \theta$ & 3.7 & 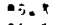 & $3: 9$ & $\because 20$ & 32.1 & 12 . & $\because \because 5$ & 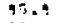 & 14.5 & $9:-:$ & נ. \\
\hline 23: & in & $? 0$ & 49.3 & a?: & A. 5. & $A=?$ & $4 \div 5$ & & & & & & & & 9. & $17 .:$ & 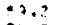 & $\because 99$ & & $9 \div .3$ & & \\
\hline $2-1$ & 17 & -1 & $7 \because 5$ & 0.7 & 91.6 & 73.4 & 91.4 & $\because .7$ & 3.9 & 79.7 & & 16 & & * & 75.6 & $\begin{array}{r}-9.9 \\
4\end{array}$ & $7+.9$ & $74_{1} 0^{\circ}$ & n.." & $\because 5$ & 7.6 & '. \\
\hline$\therefore+1$ & II & $\because 3$ & $7<.9$ & 35.4 & $7 \mathrm{~A} . ?$ & $7 \cdot 7$ & 7.9 & $74 .:$ & $\because 2$ & $\because .9$ & 7.7 & 77.7 & & 79.7 & 79.7 & $74 . ?$ & 9.9 & 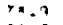 & 7.8 & $\because .4$ & 2.0 & 3. \\
\hline ta: & $T^{n}$ & $\because \because ?$ & 79.5 & -7.2 & $77 . i$ & 70.7 & 27.5 & 70.7 & 70.9 & 77.9 & 23.7 & & & $\because 9$ & $=. .7$ & $t \cdot .2$ & 6.9 & 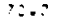 & -5 & 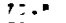 & $7: 5$ & 5.5 \\
\hline$?$ & :3 & ?: & $7 ! 1$ & $7: 5$ & $7:=$ & $7:-4$ & 71.2 & $\because: 0$ & $7 \therefore 0$ & $7: 0$ & $\therefore$ & $\because \because \div$ & $3: 9$ & & 71.7 & $7: \ldots$ & & $\because-9$ & $\because$ & 72.4 & 39 & \\
\hline ri: & ¿? & 0 & 79.6 & 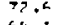 & $73 \cdot 3$ & 72.3 & 79.9 & & & & & $7 ?+7$ & & & " ? & $7 \therefore$ & .7 & $\because 4$ & & 4.5 & & \\
\hline $3 ? 1$ & in & $\geq 0$ & 63.9 & 64.1 & $54=2$ & 64.3 & 6.3 .8 & $5.4,3$ & 52.3 & 5. & 54. & $5: 0.6$ & 54. 3 & $4,6 \ldots$ & c... & ؛ & . \% & 66.7 & $A_{1} i_{1}$ & $5,4.6$ & 6.6. 7 & 56.7 \\
\hline 41 & ? & $3=0$ & 64 & $E_{t} \cdot{ }^{A}$ & $a r, 0$ & $5 .=9$ & $\therefore: 9$ & $5 . .3$ & & $55, "$ & & $65=3$ & $f=?$ & $\therefore=7$ & r.. & $\sigma_{1} \cdot r_{1},{ }^{2}$ & 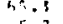 & 0.5 .3 & $5 x+?$ & $5=-1$ & 65.3 & \\
\hline & T? & 340 & 56.9 & 9.5 & 55.5 & 46.7 & $6,7,3$ & & & $5: .=$ & $65 . ?$ & $.5=2$ & & 67.7 & +5.4 & 45. & $.5 . ?$ & Gin.? & $\omega n=2$ & $6=.2$ & 15.2 & \\
\hline 391 & T? & $00 ?$ & 65,3 & ars? & $\begin{array}{l}45.7 \\
45.2\end{array}$ & $\begin{array}{l}45.2 \\
45.2\end{array}$ & 65.2 & $\begin{array}{l}450 \\
6=5\end{array}$ & 45.3 & $45 ?$ & & $45 \cdot ?$ & & & $\therefore=?$ & wa? & 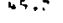 & $\leqslant x \cdot ?$ & -4.2 & 45.2 & 45.7 & 45.2 \\
\hline 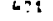 & T7 & $6: 3$ & $4 x^{2}=2$ & $45 . ?$ & 45.2 & 45.2 & 6,3 & $6=2$ & $4 ; .7$ & 65.2 & & $\Rightarrow=7$ & & & 6.7 & & & & & & & \\
\hline
\end{tabular}


AIP.

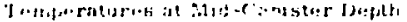

(Selocterd Tizedes)

Ths hopendix gives a graphical presentition of note temperntur:s (rise abrve ambient) at mitl-rinister lopth (z -30 roet). Sorle numberti are not shown. 


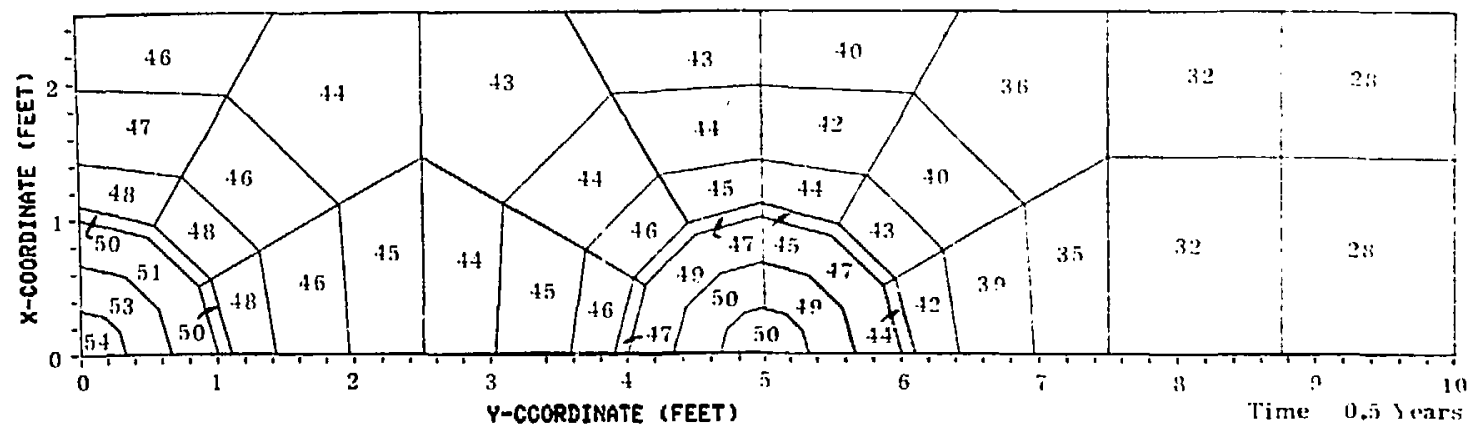

Figure 1)-1, Temperature lise Above imbient (1)eg. $C$ ) at $2,-30$ Feet

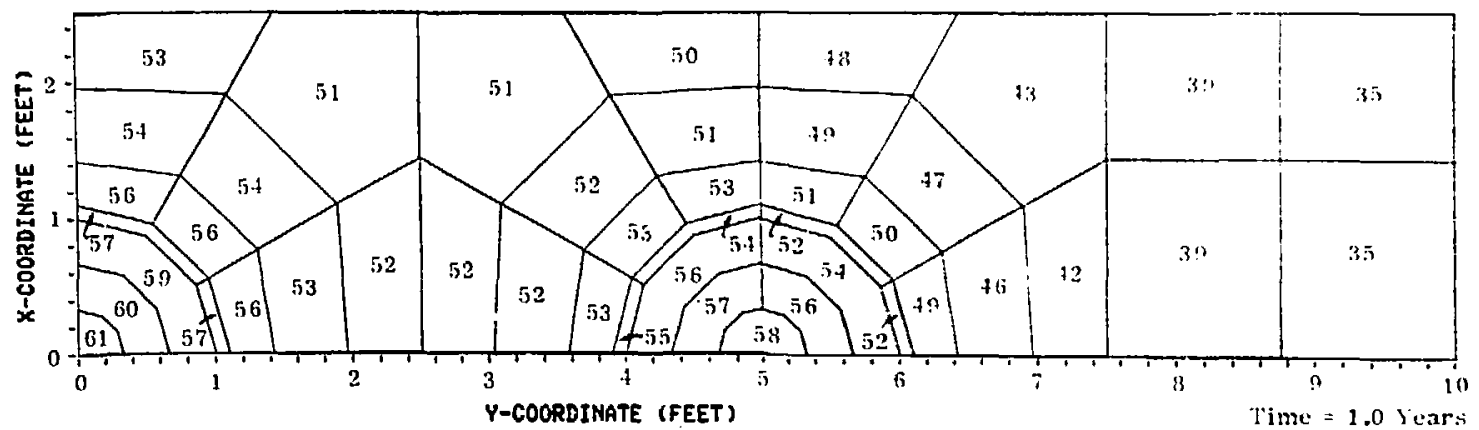

Figure 1)-2. Temperature lise Above Ambient (1)eg. (1) at $;=-30$ fuet 


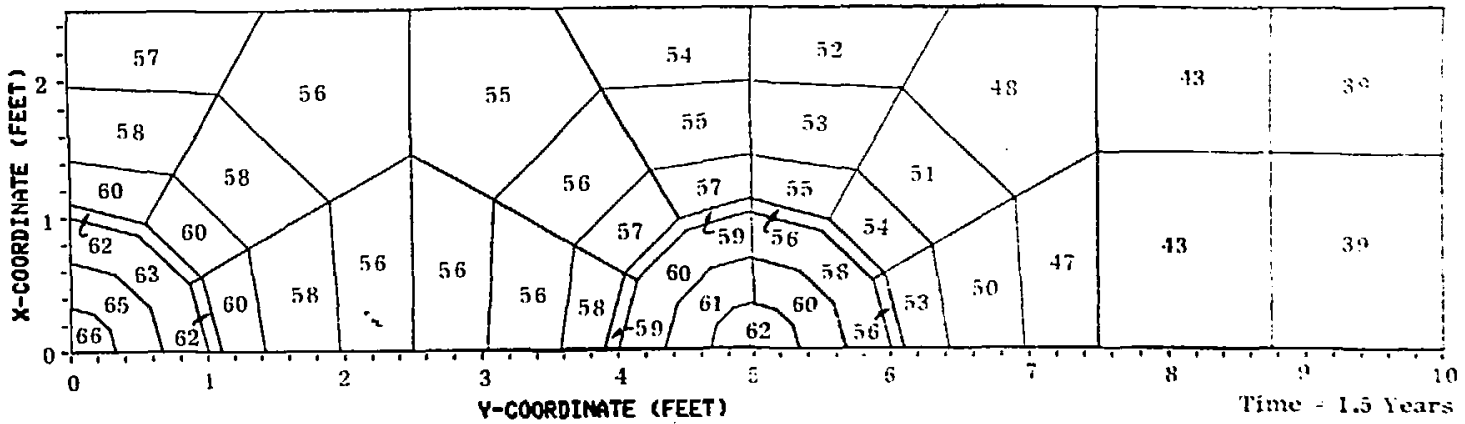

Figure 1)-3. Temperature lise Above Ainbient (Deg. () at a -30 Feet

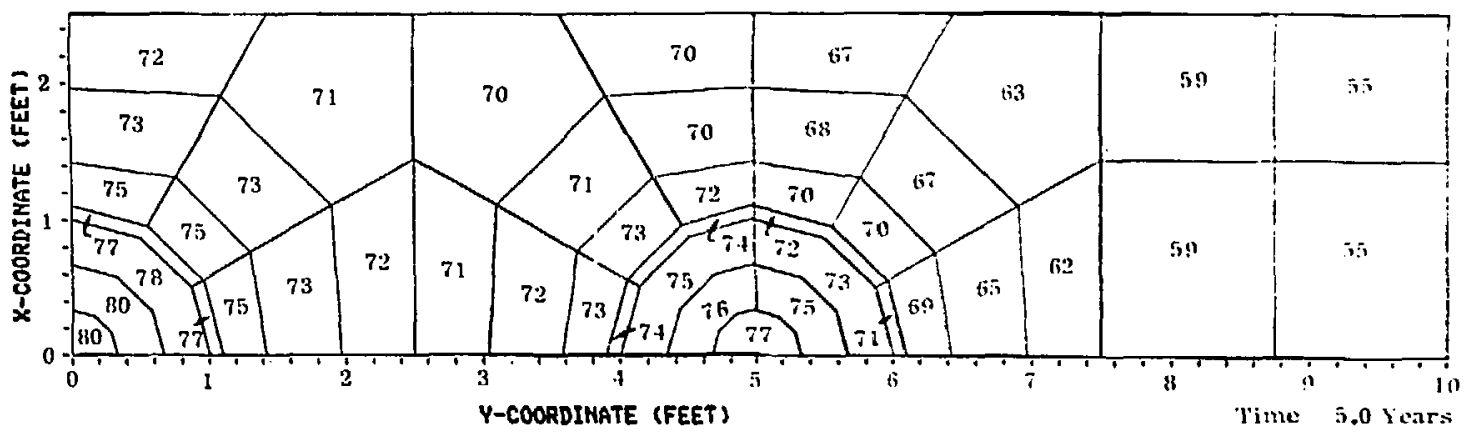

Figure 1)-4. 'Temperature lise Above Ambient (1)eg. (-) at $z-30$ leet 


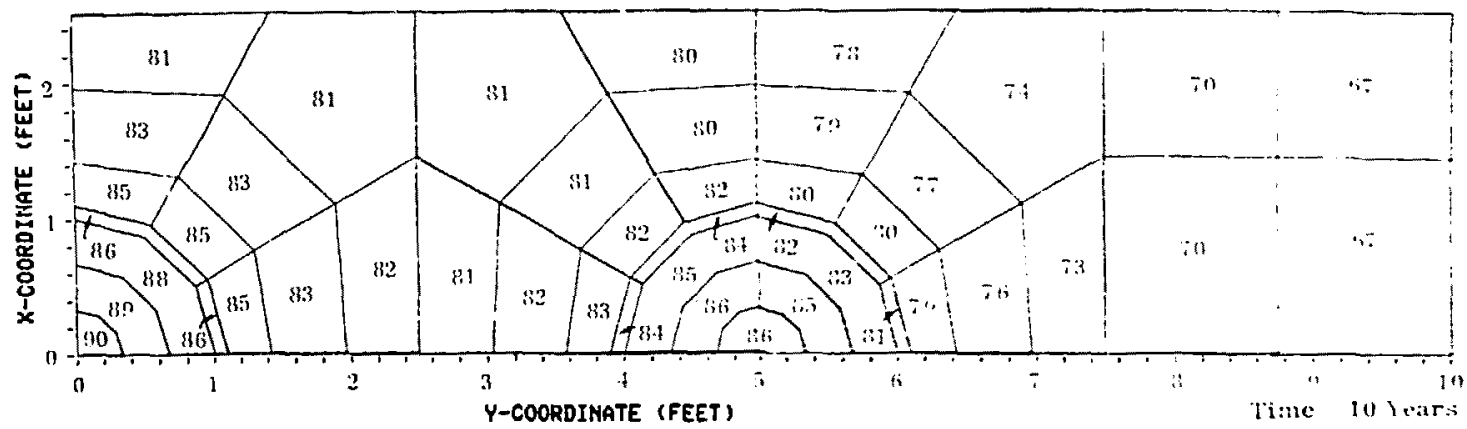

Figure 1)-5. Temperature kise lbove lmbien (1)eg- 6 ) at $:-30$ fect

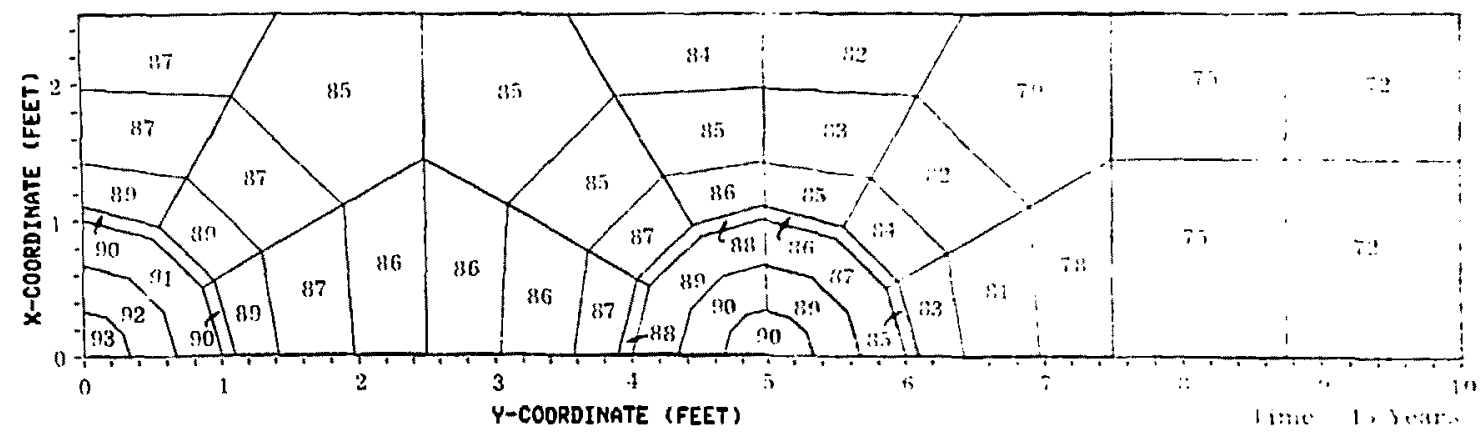

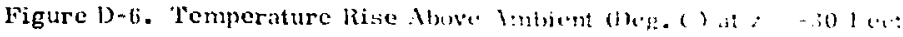




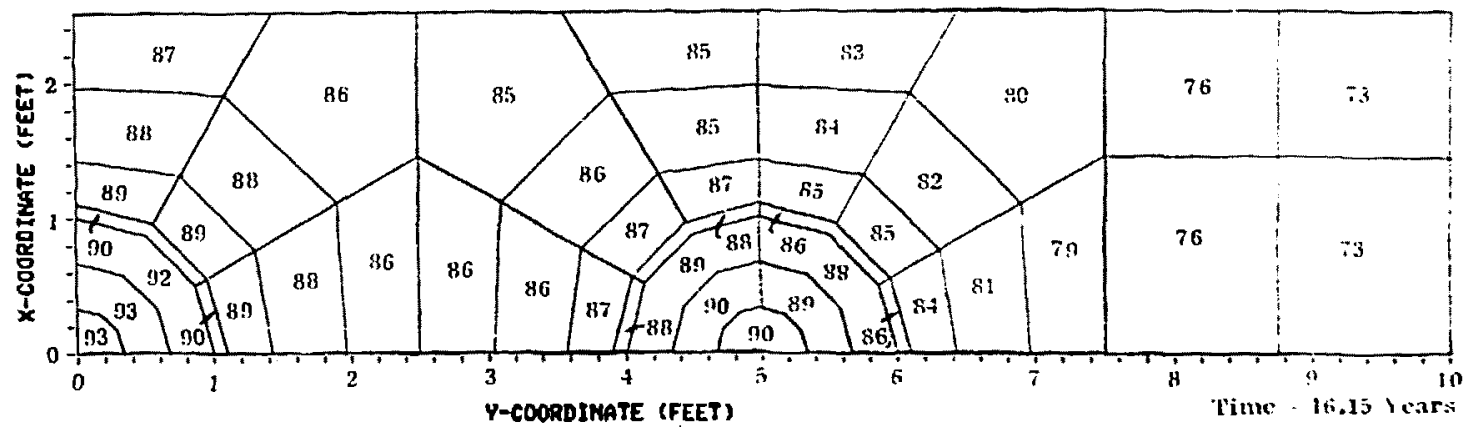

Figure 1)-7. Temperature Rise Above Anbient (1)eg. (1) at, -30 fiet 